\title{
The Solar-Stellar Connection
}

\author{
A.S. Brun, R.A. García, G. Houdek, \\ D. Nandy, M. Pinsonneault
}

Received: date / Accepted: date

\begin{abstract}
We discuss how recent advances in observations, theory and numerical simulations have allowed the stellar community to progress in its understanding of stellar convection, rotation and magnetism and to assess the degree to which the Sun and other stars share similar dynamical properties. Ensemble asteroseismology has become a reality with the advent of large time domain studies, especially from space missions. This new capability has provided improved constraints on stellar rotation and activity, over and above that obtained via traditional techniques such as spectropolarimetry or CaII H\&K observations. New data and surveys covering large mass and age ranges have provided a wide parameter space to confront theories of stellar magnetism. These new empirical databases are complemented by theoretical advances and improved multiD simulations of stellar dynamos. We trace these pathways through which a lucid and more detailed picture of magnetohydrodynamics of solar-like stars is beginning to emerge and discuss future prospects.
\end{abstract}

Keywords Sun · Stars · asteroseismology · gyrochronology · structure · rotation · magnetism $\cdot$ dynamo

Brun, A.S. and Garcia, R.A.

AIM, CEA/CNRS/University of Paris 7, CEA-Saclay, 91191 Gif-sur-Yvette, France

E-mail: sacha.brun@cea.fr and rafael.garcia@cea.fr

Houdek, G.

Stellar Astrophysics Centre (SAC), Department of Physics and Astronomy, Aarhus University, Ny Munkegade 120, DK-8000 Aarhus C, Denmark

E-mail: hg@phys.au.dk

Nandy, D.

Center of Excellence in Space Sciences India (CESSI) and Department of Physical Sciences, Indian Institute for Science Education and Research Kolkata, Mohanpur 741252, West Bengal, India

E-mail: dnandi@iiserkol.ac.in

Pinsonneault, M.

Department of Astronomy, The Ohio State University, 140 West 18th Avenue, Columbus, OH 43210, USA

E-mail: pinsonneault.1@osu.edu 
1 Solar Dynamics and Magnetism in a Stellar Context

Time-dependent stellar magnetic fields, such as that of the Sun, are generated in their interior through a magnetohydrodynamic (MHD) dynamo mechanism that involves interactions between plasma flows and magnetic fields (e.g. Moffatt 1978; Charbonneau 2010). The generated magnetic fields are manifested at the surface through starspots or polar caps, and contribute to the dynamic activity of the star. This activity governs the structure of stellar coronae, the stellar radiative flux output, and transient but energetic events such as flares (Güdel 2004; Schriiver et al. 2012). Stellar activity can also have a strong impact on the properties of stellar astrospheres which may host planets (Wood 2004). Magnetized solar-like winds extract angular momentum which drives angular momentum in low mass stars. Understanding the origin of stellar magnetism is therefore of crucial importance, and remains an outstanding problem in astrophysics.

The Sun, our nearest star, provides easy access to spatially resolved observations of magnetic activity and thus sets constraints on the dynamo origin of stellar magnetic fields. However, significant uncertainties in our current understanding of the solar dynamo mechanism motivates us to confront our theoretical ideas of magnetic field generation with the wider space parameters available from stellar activity observations (Noyes et al. 1984a; Baliunas et al. 1995; Wright et al. 2004; Giampapa 2005; Böhm-Vitense 2007; Oláh et al. 2009; Donati and Landstreet 2009; Reiners 2012, and references therein). While, in principle, different stars with different properties may host distinct classes of MHD dynamos, a good starting assumption is that a reasonable model of solar and stellar magnetism should be able to reproduce the range of stellar activity taking into account appropriate changes in the internal driving mechanisms. For example, high mass stars have shallow surface convective zones with high turbulent fluxes relative to low mass stars. Stars with different rotation rates and ages will have different magneto-convective properties (usually characterized by varying nondimensional parameters such as the Reynolds or Rossby numbers). To reduce this problem to a practical exercise, it is also useful to deal with solar-like stars (narrowing the range of spectral type considered), within which, there is substantial variation in the nature of magnetic output (Petit et al. 2008; Marsden et al. 2013). The observation and characterization of stellar convection, rotation and magnetism has undergone a recent boom with the outcome of better ground-based instruments and space-born missions. For instance, until recently chromospheric proxies such as emission in the core of CaII H \& $\mathrm{K}$ lines and white light modulation were the most commonly used techniques for characterizing stellar magnetism (Wright et al. 2004; Giampapa 2005). The development of very sensitive spectropolarimeter such as ESPaDOnS or NARVAL has helped to further improve our understanding of stellar magnetism by providing detailed magnetic maps and sometimes the surface differential rotation of the star (Donati et al. 1997; Jardine et al. 1999; Donati et al. 2006). With the success of planet transit/asteroseismology missions such as Corot, Kepler, the picture can now be refined even further (Garcia et al. 2014). We have now entered an era in which systematic coupled analyses of chromospheric activity proxies, spectropolarimetry maps and asteroseismic data is being performed. This allows us to better characterize magnetism of solar-like stars and its variability on short and relatively long terms (Berdvugina 2005; Petit et al. 2008; Morgenthaler et al. 2011a.2011b; Morin et al. 2013; Mathur et al. 2013a). The purpose of this review paper is to recap recent progress made, with the aid of asteroseismology, theory and numerical simulations, in our understanding of stellar dynamics and magnetism in solar-like stars. Other proxies, such as the time 
evolution of surface lithium abundances, can also be useful to constrain stellar dynamics but they are outside the scope of this review (for discussions see Brun et al. 1999; Talon and Charbonnel 2004; Sbordone et al. 2010; Do Nascimento et al. 2010; Spite et al. 2012, and references therein). We begin by discussing recent observational findings with a special emphasis on ensemble asteroseismology and on stellar dynamics, magnetism and cyclic activity. We then present the current theories of stellar rotational history based on 1-D models and of stellar magnetism with an introduction to dynamo theory based on 2-D kinematic dynamo models and 3-D nonlinear turbulent numerical simulations of stellar dynamo. We continue with a discussion on how theoretical models hold up against observations such as scaling laws and rotation-activity relationship (Pizzolato et al. 2003; Wright et al. 2011) and provide a critical assessment of the current understanding. Finally we conclude by proposing possible future developments in asteroseismology and stellar dynamics to better constrain solar-like star rotation and activity.

1.1 New Asteroseismic Diagnostics of Global Stellar Convection, Rotation and Magnetism

Oscillations are intrinsically damped and stochastically excited by the turbulent motion in the outer convective layers of cool stars (e.g. Goldreich and Keelev 1977; Balmforth 1992; Houdek et al. 1999; Chaplin et al. 2005; Belkacem et al. 2008; Houdek 2010). We refer to stars exhibiting such an oscillation pattern as solar-type stars hereafter. These oscillations are also present with higher amplitudes in red-giant stars (e.g. Houdek and Gough 2002; de Ridder et al. 2009; Bedding et al. 2010; (Mosser et al. 2010) (and even in mores massive B-type stars as recently shown by Belkacem et al. 2009). The frequency of maximum power scales as $\left(G M / R^{2}\right) T_{\text {eff }}^{-1 / 2}$ (e.g., Kieldsen and Bedding 1995); given the wide dynamic range in stellar surface gravities the relevant periods span the range of minutes to weeks.

The magnetic activity cycles in the Sun and other solar-like stars are often the consequence of dynamo processes induced by the interaction of three fundamental mechanisms: convection, rotation, and magnetic fields (see detailed discussion in §2). Asteroseismic observations place powerful observational constraints on each of these three processes as we will see in $\S 1.4,1.5$, and 1.6. For example, activity-cycle induced variations of pulsation properties (mode linewidth and amplitudes) in the Sun and in solar-like stars were discussed, for example, by Houdek et al. (2001) and Chaplin et al. (2007).

The long and uninterrupted high-precision photometric observations required for asteroseismology are ideal for characterizing long-term variations such as the ones induced by starspots crossing the visible disk of stars. Indeed, the dimming produced by these spots leads to a measurable modulation in the photometry with a period corresponding to the surface rotation at the active latitudes (e.g. Basri et al. 2011; García et al. 2012; Garcia et al. 2014; Lanza 2013; Walkowicz and Basri 2013; Nielsen et al. 2013; Reinhold et al. 2013; McQuillan et al. 2014) in which the spots develop. One must keep in mind that on the Sun faculae and the small-scale field around over-compensate this dimming effect, leading to a net brightening at solar maximum (Spruit 2000; Fröhlich 2012). Moreover their relative amplitude depends on how close to the limb the starspot is and to the stellar inclination angle with respect to the line of sight. Mapping the relative ratio of dimming through star spot vs. brightening through 
faculae and small-scale field changes as a function of stellar spectral type, age, rotation rate and metallicity is an emerging field, and as of this paper the overall pattern is not firmly established. Some studies have started to address the physical origins of stellar variability (e.g. Shapiro et al. 2014) and found that a transition from net brightening to net dimming should occur around an index of chromospheric activity $\log \left(R_{\mathrm{HK}}^{\prime}\right)$ of -4.9 , a value which is consistent with observations (Lockwood et al. 2007; Hall et al. 2009).

Ground based stellar variability studies have a long history, and they have traditionally focused on young and active stars. The Convection Rotation and planetary Transits (CoRoT) and Kepler space missions have contributed continuous and precise data series for large samples, which has had a dramatic impact on the quantity and quality of stellar rotation data. CoROT and Kepler have extended our understanding to large numbers of older and less active field stars (e.g. Mosser et al. 2009; Mathur et al. 2010b, 2011b; Campante et al. 2011). Asteroseismology has also extended our understanding of longer term cycles. In the solar case the number of spots in the Sun changes during the solar magnetic cycle (Hathawav 2010), introducing a modulation of the variance in the photometric observations that can be used as a proxy of the magnetic activity cycle in the Sun (Mathur et al. 2009) and in other solar-like stars (e.g. García et al. 2010; Chaplin et al. 2011a). As the temperature rises from the photosphere to the chromosphere, the cores of very strong lines, such as Ca II H (396.8 nm) and K (393.4 nm) emission lines (Leighton 1959; Wilson 1978a; Baliunas et al. 1995), develop emission features whose strength depends on the magnitude of the temperature inversion. However, seismic techniques can go further by probing structural and dynamical changes beneath the photosphere as the magnetic cycles develop. Indeed, since the dawn of helioseismology, several authors noticed that the frequency of the acoustic modes increase near the solar maximum (Woodard and Noves 1985; Fossat et al. 1987; Pallé et al. 1989; Libbrecht and Woodard 1990; Anguera Gubau et al. 1992). Nowadays, it is possible to measure variations in all the p-mode properties not only in the Sun (e.g. Chaplin et al. 2000; Jiménez-Reves et al. 2007), but also in other stars such as the F star HD49933 measured by CoRoT, in which the amplitude as well as the frequency shifts of low-degree modes change with time (García et al. 2010).

Asteroseismic measurements can also be used to extract information about convection. The $\mathrm{p}$ modes are observed as a power excess at distinct frequencies on top of a continuous Fourier spectrum. This background spectrum is likely due to the presence of convective motions at all scales with granulation playing a key role (e.g. Harvey 1985; Lefebvre et al. 2008), but may also be due to the presence of faculae (e.g. Karoff et al. 2013). Until now, neither classical observations nor one-dimensional analytical convection models could put constraints on granulation. The situation has changed with asteroseismic observations of thousands of stars thanks to the CoRoT (Baglin et al. 2006) and Kepler (Borucki et al. 2010) missions. For instance, Mathur et al. (2011a) have determined the timescale and power of granulation for around 1000 Kepler red giants at different stages of their evolution going from the red giant branch up to the clump. Moreover, the depth $d_{\mathrm{bcz}}$ of the convective zone -which is often the location used to compute a caracteristic convection overturning time e.g. $\tau_{\text {conv }} \sim d_{\text {bcz }} / v_{\text {conv }}$, with $v_{\text {conv }}$ being a characteristic convective velocity - is a region characterized by a sharp change in stratification, since it changes from (super)-adiabatic to subadiabatic, modulo the extent of convective penetration (e.g., Zahn 1991). Therefore, the sound speed undergoes an abrupt variation producing an acoustic glitch that leaves a fingerprint in the frequencies of the acoustic modes (e.g., Gough and Thompson 1988; 
Vorontsov 1988; Gough 1990) see also (Monteiro et al. 1994; Basu and Antia 1995; Houdek and Gough 2007; Christensen-Dalsgaard et al. 2011; Mazumdar et al. 2014).

1.2 The Link Between Helio- and Asteroseismology: Global Oscillations and 1-D Models

Helioseismology has been providing a wealth of high-quality information about the structure and physics of the solar interior since the first detection of the global fiveminute oscillation modes (Claverie et al. 1979). The large number of observable global solar oscillation modes, with spherical degrees up to several thousands, has allowed helioseismologists to infer with high accuracy from the measured oscillation frequency the solar sound speed and density as a function of solar radius. Such helioseismic inversion techniques make use of the fact that the displacement eigenfunctions are related to the adiabatic eigenfrequencies through a variational principle (Chandrasekhar 1963; Lynden-Bell and Ostriker 1967) leading to a linear relation between a small perturbation in the density (and squared sound speed) of a solar model and the corresponding change in the frequencies (e.g. Gough 1978, 1984b). Before the results of the Sudbury Neutrino Observatory (SNO) experiment (see further down), the solar neutrino problem, together with the high-quality helioseismic data collected from space, indicated clear problems in our understanding of either basic physics or the structure of the solar interior. In response, there was a major sustained effort to improve solar models; for a review see e.g. Christensen-Dalsgaard (2004) and references therein. The rather good agreement $(<1 \%)$ between the Sun and 1-D solar models computed in the late 1990s (Christensen-Dalsgaard et al. 1996; Brun et al. 1998, 1999) was, however, degraded if the solar photospheric abundances reported by Asplund et al. (2004) were adopted. The problem still holds even after the revision to a slightly higher value of metalicity by Asplund et al. (2009). We refer to Christensen-Dalsgaard and Houdek (2010), Villante et al. (2014) and chapter 2 (e.g., Basu et al. 2014) of the ISSI book ?? for a recent discussion on helioseismology constraints on metallicity/abundances.

Still, the high-quality helioseismic data has led to many fundamental achievements in solar physics such as inverting the internal solar angular-velocity profile or helping to disentangle the long-standing solar neutrino problem. Seismic inversions have revealed that the non-uniform, surface differential rotation extends all the way to the base of the convective envelope and becomes almost uniform (e.g. solid body rotation) in the radiative interior (e.g. Brown and Morrow 1987; Thompson 1990; Thompson et al. 2003; García et al. 2004; Mathur et al. 2008; Eff-Darwich and Korzennik 2012). The inferred radial interior density and sound-speed profiles also place strong constraints on the standard solar model and consequently on the theory of stellar structure and evolution. Since the neutrino fluxes predicted by solar models could not be explained by standard zero mass neutrino physics, particle physicists had to consider massive neutrinos and their associated flavor oscillations (e.g. Turck-Chièze et al. 2001). Thanks to the SNO experiment measurements (Ahmad et al. 2001) the scientific community has since confirmed, by measuring both charged and neutral currents, that the original deficit of electronic neutrinos is due to their transition to another neutrino flavor as they travel from the solar core to Earth's detectors. Another important issue, which has again become topical very recently by analyzing the high-quality data from the Heliospheric and Magnetic Imager (HMI) on NASA's Solar Dynamics Observatory (SDO), is the solar oblateness (Kuhn et al. 2012) and the consequent distortion of the 
Sun's gravitational field which may account, in part, for the remaining $0.2 \%$ discrepancy in the precession of Mercury's orbit (e.g. Schou et al. 1998; Gough 2012). Detailed discussions about what we have learnt from helioseismology and how helioseismology can help to address the remaining problems in solar physics can be found in recent reviews by Christensen-Dalsgaard (2004) and Gough (2013) and all the chapters of the ISSI book ??

That stellar variability provides deep insights into stellar structure has been known since the beginning of the 20th century (e.g. Shapley 1914). In nonradial oscillators such as the Sun, the numerous pulsation modes with distinct frequencies encode detailed information about their interior structure. The Sun oscillates in approximately 10 million distinct oscillation modes and with the help of space-borne instruments we are now able to measure some 6000 individual modes and up to several million modes via so-called ridge fitting. The amplitudes of solar-like oscillations, however, are small compared to those observed in classical pulsators, such as Cepheids or Delta Scuti stars, and their frequencies can also be quite high ( 5 minutes in the solar case). Therefore only the latest generation of instruments have been able to detect solar-like oscillations in stars other than the Sun.

The first indication of excess acoustic power of solar-like oscillations in other stars with a frequency dependence similar to the Sun was reported by Brown et al. (1991) for the F5 star Procyon A ( $\alpha \mathrm{CMi}$ ), for which the first unambiguous detection of solar-like oscillations was reported by Martić et al. (1999) from the ground, as well as by Schou and Buzasi (2001) using data collected by the WIRE spacecraft. These results were confirmed later by Mosser et al. (2008). The first (unconfirmed) detection of individual peaks in the acoustic power spectrum from high-precision time-resolved spectroscopic observations was published for the G0 star $\eta$ Boo by Kieldsen et al. (1995), but it was not until 2003 that an unambiguous confirmation was established by (Carrier et al. 2003; Kjeldsen et al. 2003).

High-degree oscillation modes will not be accessible in other stars in the foreseeable future because they require spatially resolved variability data. However, the observable low-degree modes in distant stars still provide rich information about the internal structure and dynamics of a large number of solar-like stars at different evolutionary stages, as demonstrated recently by the results obtained by the CoRoT and Kepler missions.

Additional projects, such as the Sun-in-time (Güdel 2007) or the Sun as a star (Giampapa 2005), try to characterize the Sun by comparing its property to solar-like stars at various evolutionary stages. In particular, the understanding of solar rotation, convection, variability and magnetic activity in a stellar context allows to better constraint theory by deriving key scaling laws as a function of age, rotation, chemical abundances and mass of those various physical processes. We defer to $\S 1.4$ to 1.6 for discussions of the relevant observations and to $\S 2$ for the description of the current theoretical understanding.

Returning to asteroseismic diagnostic, even with the limited seismic information of low-degree oscillation modes, some theoretically important properties have become accessible, such as the gross structure of the energy-generating core and the extent to which it is convective, and a large-scale core to envelope contrast in the angular velocity of evolved stars (e.g. Mosser et al. 2012; Deheuvels et al. 2012; Mathur et al. 2012; García 2013, and references therein). Such information will be of crucial importance for checking, and then calibrating, the theory of the structure and evolution of stars. 
One of the many interesting applications of utilizing low-degree modes for measuring various aspects of the structure in distance stars is the measurement of rapid variations in the stellar background, brought about by, for example, the rapid variation in the acoustic cutoff frequency at the base of the surface convection zone or the variation in the first adiabatic exponent in the helium ionization zones. The design and analysis of low-degree seismic signatures of the structure of spherical stars is therefore of central importance to asteroseismic diagnosis, and various research groups have been working on improved seismic diagnostic techniques (e.g. Houdek and Gough 2007, and references therein). First applications of low-degree seismic diagnostic techniques have been carried out recently on 19 solar-type stars observed by the Kepler mission (Mazumdar et al. 2014).

However our poor understanding of the physics of the stellar surface layers causes a major problem in the analysis of global solar-like oscillation, because the properties of the near-surface layers have a major impact on oscillation frequencies. Typical stellar structure calculations treat the superficial layers with simplified atmosphere models and the stratification of the superadiabatic region by means of a local, time-independent mixing-length approach (e.g. Böhm-Vitense 1958). The effects of the (turbulent) Reynolds stresses in both the hydrostatic equilibrium and pulsation calculations are essentially always ignored. Linear pulsation calculations typically adopt the adiabatic approximation and ignore the momentum flux perturbations (turbulent pressure fluctuations). However, nonadiabatic effects and the fluctuations of the turbulent fluxes (heat and momentum) do modify the modelled pulsation eigenfunctions and consequently also the oscillation frequencies (e.g. Gough 1984a; Balmforth 1992; Houdek 1996, 2010). For a "standard" solar model, such as Model S (Christensen-Dalsgaard et al. 1996), the frequency differences, scaled with the mode inertia, between the solar and model frequencies are dominated by the near-surface effects and are predominantly a function of frequency alone. Differences between solar frequencies observed with the GONG instruments and adiabatically computed Model $\mathrm{S}$ frequencies are illustrated by the the symbols (plusses) in the left panel of Figure 1 (colours indicate different spherical degrees). The increase of the frequency residuals with oscillation frequency depends on the modelling details of the functional form of the acoustic cutoff frequency (as it affects the acoustic potential) with radius in the near-surface layers. In the Sun the acoustic cutoff frequency is about $5.5 \mathrm{mHz}$.

Sophisticated 3-D hydrodynamical simulations of stellar convection have been used to estimate the effect of turbulent pressure in the equilibrium model (the mean Reynolds stress). For example, Rosenthal et al. (1995) investigated the effect on adiabatic eigenfrequencies of the contribution that the turbulent pressure makes to the mean hydrostatic stratification. They examined a hydrodynamical simulation by Stein and Nordlund (1991) of the outer $2 \%$ by radius of the Sun, matched continuously in sound speed to a model envelope calculated, as in a "standard" solar model, with a local mixing-length formulation. The resulting frequency shifts of adiabatic oscillations between the simulations and the "standard" solar reference model, Model S, are illustrated in the right panel of Figure 1. The frequency residuals behave similarly to the data (plus symbols) in the left panel of Figure 1 though with larger shifts at higher oscillation frequencies.

The effects of nonadiabaticity and convection dynamics on the pulsation frequencies were, for example, studied by Balmforth (1992), Rosenthal et al. (1995) and Houdek (1996). In these studies the nonlocal, time-dependent generalization of the mixinglength formulation by Gough $1977 \mathrm{a}$ 1977b) was adopted to model the heat and momentum flux consistently in both the equilibrium envelope model and in the linear 


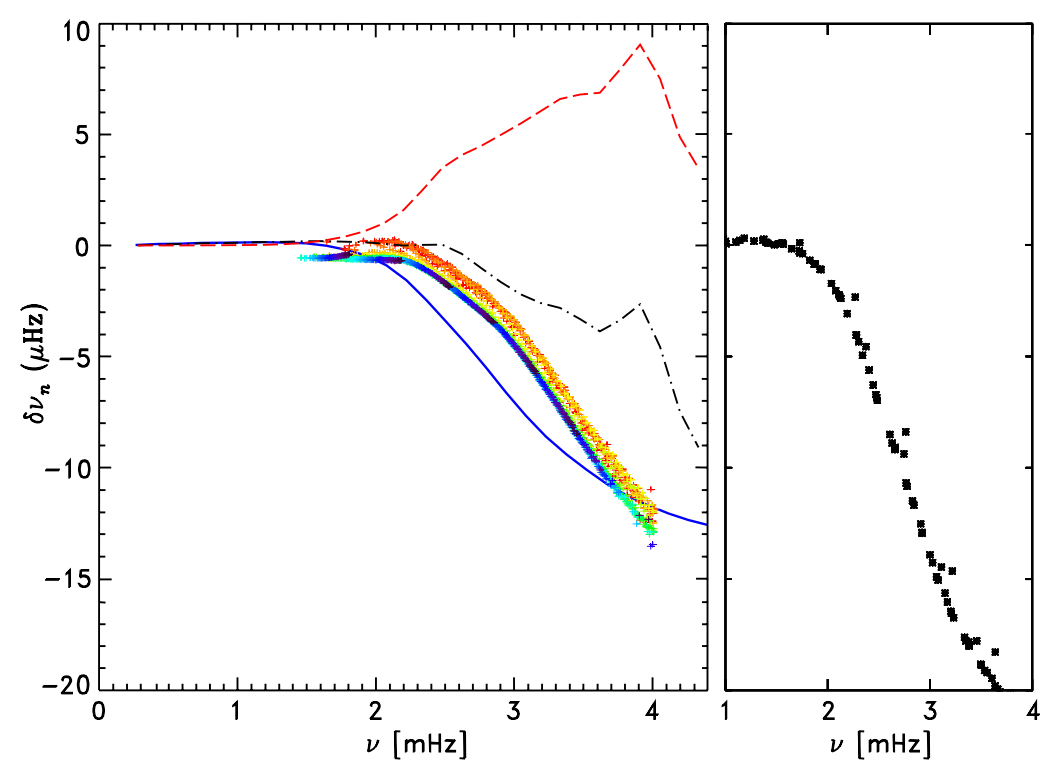

Fig. 1 Figure 1: Frequency residuals between the Sun and solar models as a function of frequency. Left: scaled differences between observed GONG frequencies and adiabatically computed frequencies of the "standard" solar Model S. Different colours indicate different degrees of the oscillations modes (adapted from Christensen-Dalsgaard et al. 1996). The curves show frequency residuals between models computed with a nonlocal time-dependent and a "standard" mixing-length formulation for convection: The solid (blue) curve shows the adiabatic residuals cause by the Reynolds stress in the mean model. The dashed (red) curve is the frequency shift caused by nonadiabaticity and convection dynamics. The overall frequency shift is plotted by the dot-dashed curve (adapted from Houdek 1996). Right: Scaled adiabatic frequency differences between a model for which the near-surface layers were represented by a hydrodynamical simulation, and the "standard" solar Model S (adapted from Rosenthal et al. 1995).

nonadiabatic stability analysis. The outcome of such calculations is shown in the left panel of Figure 1 (Houdek 1996, 2010) by the dot-dashed curve. Similar as for the hydrodynamical simulations the effect of the Reynolds stresses in the mean structure decreases the adiabatic frequencies (solid curve) with a maximum deficit of about $12 \mu \mathrm{Hz}$, which is much smaller than the deficit of about $24 \mu \mathrm{Hz}$ from the hydrodynamical simulations (symbols in the right panel).

The effects of nonadiabaticity and convection dynamics (dashed curve in the left panel), however, lead to an increase of the mode frequencies by as much as $\sim 9 \mu \mathrm{Hz}$, nearly cancelling the downshifts from the effect of the Reynolds stress (turbulent pressure) in the mean structure (solid curve in the left panel), leading to the overall effect indicated by the dot-dashed curve in the left panel. If the positive frequency shifts between models computed with and without nonadiabatic effects and convection dynamics (dashed curve in the left panel) are interpreted as the nonadiabatic and momentum flux corrections to the oscillation frequencies then their effects are to bring the frequency residuals of the hydrodynamical simulations (right panel) in better agreement with the data (plus symbols in the left panel of Figure 1). If we were to understand the reason why the semi-analytical, nonlocal convection model underestimates the effect of the Reynolds 
stress in the mean model on the adiabatic eigenfrequencies, we would come closer to a self-consistent explanation for the still unsolved problem of reproducing theoretically the observed high-frequency oscillations in the Sun and in sun-like stars. Currently only simple procedures for estimating the near-surface frequency corrections (Kjeldsen et al. 2008) are adopted by the community (e.g. Mathur et al. 2012). This simple procedure is based on the calculations of a model, in which the frequency shifts are scaled by a power-law in frequency (Christensen-Dalsgaard and Gough 1980). A physically more realistic description of the surface effects with reliable applications to solar-like stars is therefore of imminent importance for the whole community to determine more reliable radii and ages in distant stars from the observed mean large frequency separation and small frequency separation, for they depend crucially on the near-surface frequency corrections (e.g. Christensen-Dalsgaard and Gough 1980; Houdek 2010).

\subsection{Asteroseismic Scaling Relations}

With the advent of space missions, asteroseismologists studying solar-like stars started to apply techniques originally developed for Sun-as-a-star observations to other stars. With only one or two solar-like stars per target field observed by CoRoT (e.g. HD 49933 , HD 181420 and HD 181906, Appourchaux et al. 2008; Barban et al. 2009; García et al. 2009, respectively), it was possible to do a full analysis of the power spectrum, determine the individual p-mode frequencies and, after combining the seismic observables with the spectroscopic ones (e.g. Bruntt 2009), perform a full modeling of each target using stellar evolution codes (e.g. Mathur et al. 2013b). Later on, with the study of red giants in the CoRoT exoplanet field and the arrival of Kepler, asteroseismologists faced a new challenge: the need to analyze massive samples of thousands of stars with high-quality light curves. The combination of the high data volume and the surface effects above made manual analysis impractical. As a result, seismologists turned to characterizing the pulsation frequency patterns with a measurement of the typical frequency of oscillation and the typical frequency spacing between carefully selected and classified modes. To do this for large sample, automatic pipelines were developed in order to extract these mean seismic observables: $\Delta \nu$ and $\nu_{\max }$ (e.g. Huber et al. 2009; Mosser and Appourchaux 2009; Hekker et al. 2010; Mathur et al. 2010a).

The large frequency spacing, $\Delta \nu_{\ell}(n)=\nu_{n, \ell}-\nu_{n-1, \ell}$, is proportional to the sound travel time in the cavity in which the mode propagates and, therefore, it is proportional to the density inside the star: $\Delta \nu \approx\left(M / R^{3}\right)^{1 / 2}$. This relation shows a very good agreement with modeling (e.g. Ulrich 1986) and can be scaled from the solar values as (Kjeldsen and Bedding 1995):

$$
\Delta \nu \approx \Delta \nu \odot\left(\frac{M}{M_{\odot}}\right)^{1 / 2}\left(\frac{R}{R_{\odot}}\right)^{-3 / 2},
$$

in which $\Delta \nu_{\odot}=135.1 \pm 0.1 \mu \mathrm{Hz}$, as derived by Huber et al. (2011) using 111 VIRGO subseries of 30-day, each spanning from 1996 to 2005 and analyzed in the same way as the Kepler data. More recent work (White et al. 2012; Mosser et al. 2013) based on numerical simulations suggests the need for small correction terms.

In 1991, Brown et al. suggested that the frequency at the maximum power of the p-mode bump, $\nu_{\max }$, should scale with the acoustic cutoff frequency (see also Belkacem et al. 2011), and following Kjeldsen and Bedding (1995) it could be scaled from the solar values according to: 


$$
\nu_{\max } \approx \nu_{\max , \odot}\left(\frac{M}{M_{\odot}}\right)\left(\frac{R}{R_{\odot}}\right)^{-2}\left(\frac{T_{\mathrm{eff}}}{T_{\mathrm{eff}, \odot}}\right)^{-1 / 2}
$$

in which $T_{\text {eff }, \odot}=5770 \mathrm{~K}$, and $\nu_{\max , \odot}=3090 \pm 30 \mu \mathrm{Hz}$ (Huber et al. 2011). This constraint is less strongly motivated by theory and requires empirical guidance.

Therefore, from equations (1) and (2), one can obtain the stellar mass and radius as a function of the seismic variables $\nu_{\max }$ and $\Delta \nu$ - assuming that $T_{\text {eff }}$ is known independently of evolutionary models (e.g. Miglio et al. 2009):

$$
\begin{gathered}
R \approx R_{\odot}\left(\frac{\Delta \nu_{\odot}}{\Delta \nu}\right)^{2}\left(\frac{\nu_{\max }}{\nu_{\max , \odot}}\right)\left(\frac{T_{\mathrm{eff}}}{T_{\mathrm{eff}, \odot}}\right)^{1 / 2}, \\
M \approx M_{\odot}\left(\frac{\Delta \nu \odot}{\Delta \nu}\right)^{4}\left(\frac{\nu_{\max }}{\nu_{\max , \odot}}\right)^{3}\left(\frac{T_{\mathrm{eff}}}{T_{\mathrm{eff}, \odot}}\right)^{3 / 2} .
\end{gathered}
$$

It has also recently been shown (e.g. Stello et al. 2009; Hekker et al. 2009; Mosser et al. 2010; Hekker et al. 2011) that solar-like oscillations in main-sequence stars follow to a good approximation the following relation:

$$
\Delta \nu \approx \Delta \nu \odot\left(\frac{\nu_{\max }}{\nu_{\max , \odot}}\right)^{b}
$$

where $b$ has often values around 0.75 . Figure 2(top) shows the relation $\Delta \nu$ versus $\nu_{\max }$ for 1700 stars from the main sequence phase (black diamonds at the right hand side) to the red clump phase (red triangles appearing in a diagonal branch between 20 to $50 \mu \mathrm{Hz}$ in the bottom panel) observed by the Kepler satellite. Although the relation appears to be constant for all of these stars, several authors (Mosser et al. 2010; Huber et al. 2010) have suggested that the slope is different between red-giant and main-sequence stars. To enhance such a difference, we have subtracted the luminosity dependence by raising $\nu_{\max }$ to the power of $b=0.75$. A fit to the residuals below and above $\nu_{\max }=$ $300 \mu \mathrm{Hz}$ - which roughly marks the transition from low-luminosity red giants to subgiants results in a steeper slope with increasing $\nu_{\max }$. It is important to note that for $\nu_{\max }$ close to the solar value, for example, the use of a power-law relation calibrated to red-giant stars would lead to an underestimation in $\Delta \nu$ by $\sim 10 \%$.

Very precise surface-gravity values, $(g)$, - with uncertainties much less than 0.05 dex - can also be inferred directly from the global seismic parameters and from the effective temperature: $g \approx \nu_{\max } \sqrt{T_{\text {eff }}}$ (e.g. Morel and Miglio 2012; Thygesen et al. 2012; Hekker et al. 2013). This is very important because the seismic $\log g$ values can be used as an independent fixed parameter in the spectroscopic analysis to compute the effective temperatures in an iterative way (Silva Aguirre et al. 2012). Indeed, both parameters are correlated and cannot be determined independently from the spectra. Therefore, the combination of a seismic $\log g$ and a spectroscopic $T_{\text {eff }}$ can reduce the uncertainties in both parameters. CoRoT and Kepler stars with asteroseismic surface gravities will be used as calibration stars for the GAIA mission (Creevey et al. 2013). 


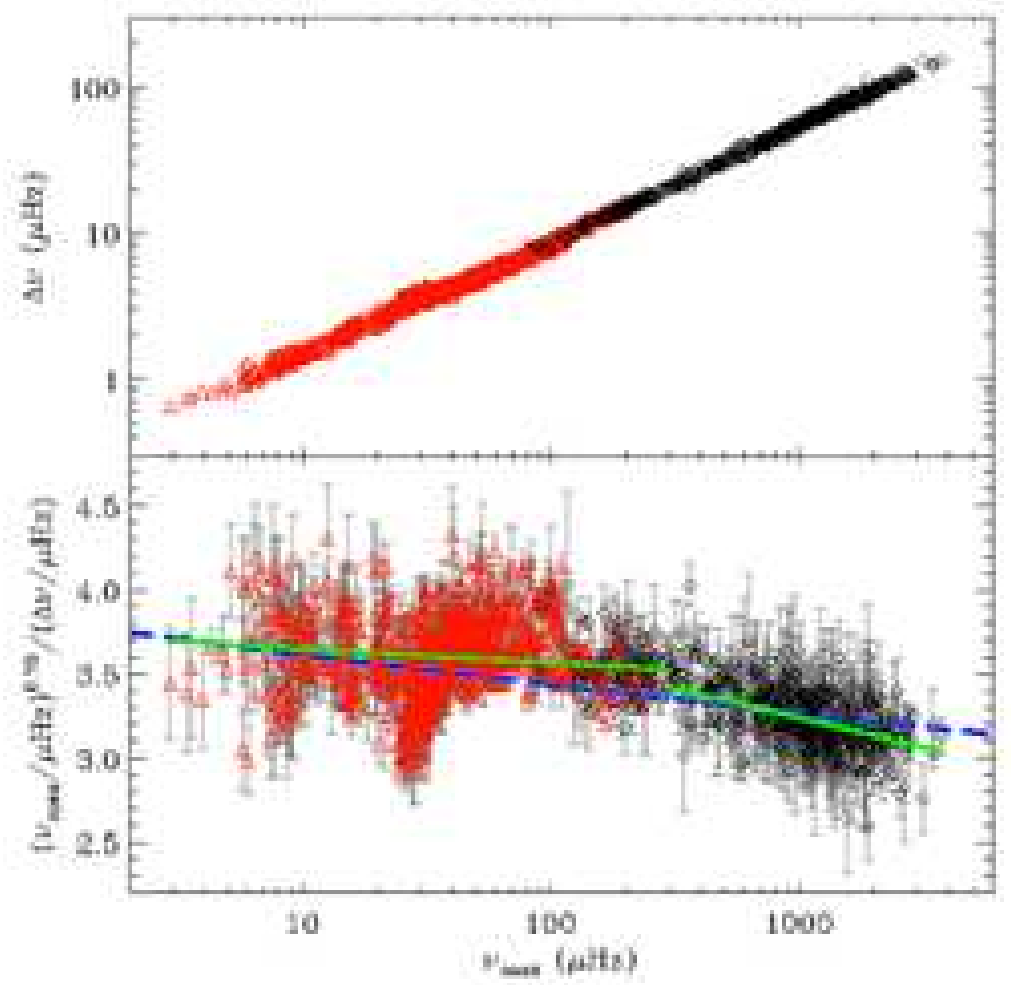

Fig. $2 \Delta \nu$ versus $\nu_{\max }$ (top panel) for 1700 stars observed by Kepler taken from Huber et al. (2011). The red triangles show stars observed in long cadence (red giants), while black diamonds are stars observed in short cadence (solar-like stars). In the bottom panel we have shown the same relation but after removing the luminosity dependence by raising $\nu_{\max }$ to the power of 0.75 . Green lines show power law fits to the $\Delta \nu-\nu_{\max }$ relation for two different intervals of $\nu_{\max }$. The blue dashed line shows the relation of Eq. [5] derived using both red giants and main-sequence stars (Stello et al. 2009).

1.4 Stellar Convection and the Background Signal in Oscillation Spectra

Solar-like pulsating stars are those in which their acoustic modes are excited by turbulent motions occurring in their convective outer layers (e.g. Goldreich and Keelev 1977; Balmforth 1992; Houdek et al. 1999; Chaplin et al. 2005; Belkacem et al. 2008). The first observations of granulation are quite old (Herschel 1801), but it is not until 1933 that their turbulent origin was unveiled (Siedentopf 1933).

In solar-like stars, exhibiting outer convective envelopes, the granules evolve with time producing small photometric fluctuations. Therefore, the analysis of stellar light curves in the Fourier domain shows a continuous "background" on top of which the acoustic modes are superimposed (see some examples of main sequence stars, subgiants and red giants in Kallinger et al. 2010; Chaplin et al. 2010; Campante et al. 2011; Ballot et al. 2011; Mathur et al. 2011b; Metcalfe et al. 2012; Mathur et al. 2013b).

The first successful attempt to model this continuous background was done by Harvey (1985). He approximated each scale of the convective motions (granulation, 
supergranulation...) by an exponentially decaying time function corresponding to the autocovariance of its time evolution with two parameters: the characteristic time of the granules and the total brightness fluctuations associated with them (see Mathur et al. 2011a, for a complete review of the different formulations commonly used in asteroseismology). Moreover, it has been demonstrated very recently that the properties of the granulation and the global seismic variables are related (e.g. Chaplin et al. 2011b; Mathur et al. 2011a). It is also important to notice that in some stars another feature is visible close to the p-mode envelope that has been related with the presence of faculae in the star (Karoff et al. 2013, and references therein). Recent theoretical work by Samadi et al. (2013) has also tried to explain the observed relationship between granulation amplitude in the oscillation spectrum and stellar spectral type.

In the context of stellar dynamics, putting observational constraints in the convection inside stars is very important because convection is one of the major ingredients to understand stellar magnetism and dynamo processes. Therefore, precise time domain measurements can offer direct constraints on the granulation characteristics as well as the precise determination of the convective outer envelope. Recently, using Kepler data, it was for example possible to calibrate the mixing-length parameter $\alpha_{\mathrm{MLT}}$ used in classical 1-D treatment of convection, and to show that the solar value, usually used for generating grids of stellar models (Ekström et al. 2012) is overestimated (Bonaca et al. 2012). Likewise granulation data can put constraints on local (Magic et al. 2013) and global (Miesch et al. 2012) stellar convection simulations, that could in turn help us to characterize stellar oscillations, and convection parameters such as the mixing length (Trampedach and Stein 2011). This important by-product of asteroseismology studies can help to better constrain convective heat transport in 1-D stellar models, and hence to constrain the stellar radius which depends on $\alpha_{\mathrm{MLT}}$.

Asteroseismology can also provide important information on the depth of convective envelopes. At the base of the surface convection zone, there is an abrupt change of stratification (going from adiabatic to subadiabatic) which impacts the sound speed and density profiles (see the pionnering work on the base of the solar convection by (Christensen-Dalsgaard et al. 1991). By using appropriate seismic diagnostic techniques it is then possible to infer the extent of the convective zone of solar-like stars (Gough 1990; Ballot et al. 2004; Houdek 2004; Houdek and Gough 2007). This approach has now been successfully applied to Kepler data for field stars (Mazumdar et al. 2014).

Complementary to the analysis of asteroseismic data, numerical simulations to model and describe the turbulent state of stellar convective enveloppes or cores have been pursued. Several groups have undergone a systematic study of the global properties of stellar convections and their associated mean flows (differential rotation and meridional circulation). We refer to $\S 2$ for a more detailed discussion of numerical simulations of rotating and magnetized convective stars.

\subsection{Stellar Rotation and Spin Down}

All stars rotate, and understanding their rotation history is key to our overall understanding of their dynamics. The angular momentum evolution of stars is an initial value problem, and a wide range of rotation rates are observed in the youngest protostars (see for example Rebull et al. 2006, for the Orion Nebula Cluster). The relatively slow rotation of such stars (Vogel and Kuhi 1981) demands efficient angular momentum loss 
from the infalling material during the hydrodynamic collapse phase, probably through strong magnetic interactions (Shu et al. 1994). Gaseous accretion disks persist around protostars for up to $12 \mathrm{Myr}$ (Bell et al. 2013), and the exchange of angular momentum between them and their disks can dramatically modify their evolution. In the limit of strong magnetic interaction, star-disk coupling can regulate stellar rotation, preventing protostars from spinning up as they contract (Koenigl 1991; Keppens et al. 1995) but see also (Matt et al. 2012). The early angular momentum evolution of stars is therefore a window into the star and planet formation process. Stars also reach the main sequence with a range of rotation rates. This can cause star-to-star differences in the degree of rotationally induced mixing (see Pinsonneault 1997, for a review). Once on the main sequence, the surface rotation of stars responds both to internal angular momentum transport and to angular momentum loss from magnetized solar-like winds. There are two strong practical applications that result:

- 1) We can use the observed angular momentum evolution as a test of stellar physics. Many instabilities and physical processes such as dynamo action, mixing, large scale flows are directly connected to the rotational rate of stars. Stars differ in their thermal structure, rotation rates, and lifetimes; we therefore have natural laboratories for testing physical models. This is especially important for our understanding of solar-like winds, as angular momentum loss driven by them is a central driver of angular momentum evolution in low mass stars.

- 2) With calibrated models we can then use rotation as a stellar population diagnostic. Weber and Davis (1967) explained the slow solar rotation as a consequence of angular momentum loss from a magnetized solar-like wind. They predicted $\mathrm{d} J / \mathrm{d} t \sim \Omega^{3}$, which implies $\Omega \sim t^{-0.5}$ in the asymptotic limit. Skumanich (1972) confirmed this time dependence in rotation, chromospheric activity, and lithium depletion in open cluster stars. Rotation is therefore a potential age indicator, provided both that it can be measured and that the rotation-mass-age relationship can be calibrated.

Both our observational knowledge and the sophistication of our theoretical models have advanced considerably since this pioneering work. We therefore briefly summarize the observational picture (see Figure 3) before proceeding to the theoretical picture that has emerged. Rotation rates can be inferred spectroscopically from Doppler line broadening. Such measurements, however, suffer from projection effects $(v \sin i$ is what is measured) and become difficult when the rotational broadening becomes comparable to or smaller than other sources, such as microturbulence or thermal broadening. Rotation velocity measurements are therefore primarily used for young and massive stars, and through the 1980s and early 1990s most rotation data was spectroscopic. In a pioneering paper, Stauffer and Hartmann (1987) demonstrated that cool stars in the Pleiades arrived on the main sequence with a wide range of rotation rates, which created three significant problems for simple angular momentum evolution models. Extremely large torques would be predicted for rapid rotators (Pinsonneault et al. 1990), and an unsaturated wind law would not permit the survival of high rotation rates onto the main sequence. Magnetized solar-like winds are too inefficient to spin down young stars to the observed rates for the slowest rotators, requiring an additional mechanism for extracting angular momentum in the pre-main sequence. Finally, once on the main sequence, there is a rapid initial drop in rotation rate, followed by a pause where surface rotation changes little; older stars then resume spinning down at the predicted asymptotic rate. This is evidence for core-envelope decoupling 
(MacGregor and Brenner 1991; Krishnamurthi et al. 1997) on timescales of tens to hundreds of Myr (see Denissenkov et al. 2010; Gallet and Bouvier 2013, for recent discussions). The observed pattern is therefore richer than a simple power-law relationship, requiring both more sophisticated theory and stronger empirical constraints.

Star spots cause periodic brightness modulations as they transit across the visible disk. Dedicated time domain studies of stars can therefore measure rotation periods. Rotation period measurements do not suffer from inclination effects (although they require a favorable inclination angle, e.g. not pole-on, to produce a signal.) There are, however, significant observational selection effects that favor the detection of short period systems, which also tend to be high amplitude. There has been an enormous increase in the quantity and quality of stellar rotation period data. In particular, the advent of large surveys designed to search for extrasolar planets has yielded very large data sets of stellar rotation periods as a natural byproduct for both field stars (Hartman et al. 2011) and open clusters stars. Examples of the latter include the extensive MONITOR program (Aigrain et al. 2007) (see Moraux et al. 2013, for the most recent results), the SuperWASP data for the Hyades and Praesepe (Delorme et al. 2011) and the large (Hartman et al. 2009, 2010) M37 and Pleiades data sets. The Kepler and CoRoT missions reach lower amplitudes and can detect stars with longer rotation periods, pushing stellar rotation measurements into the solar regime (Basri et al. 2011; Garcia et al. 2014; Lanza 2013; Walkowicz and Basri 2013; Nielsen et al. 2013; Reinhold et al. 2013; McQuillan et al. 2014). With the aid of these samples, a standard empirical picture of angular momentum evolution has emerged, with the following basic ingredients.

Stars are born with a range of rotation rates, and they would be expected to spin up as they contract and approach the main sequence. Through protostar-disk interactions some stars lose additional angular momentum in the first $\sim 10 \mathrm{Myr}$, widening the observed distribution. The slow rotator population cannot be generated from the young star forming regions, as noted above, but is already in place in the $13 \mathrm{Myr}$ old system H Per (Moraux et al. 2013). This confirms the hypothesis that protostar-disk interactions are important for stellar angular momentum evolution. The distribution of rotation rates is initially broad and narrows with age, which is a natural consequence of a torque that increases more than linearly with rotation rate. This convergence process is most rapid for solar mass stars and the timescale for spin down increases with decreased mass. This is usually interpreted as evidence for mass-dependent saturation in the angular momentum loss rate, typically parameterized with a Rossby scaling (rotation period relative to the convective overturn timescale.) Core-envelope decoupling is required to explain young slow rotators, with a time scale that increases with decreased mass (Denissenkov et al. 2010; Delorme et al. 2011). There is no break or discontinuity in the observed spin down behavior at the fully convective boundary, which is surprising given dynamo model predictions. New data sets in older solar analogs in Kepler stars will provide tests of the mass and composition dependence of rotation in old dwarfs, which is currently lacking. Field star studies will need to consider the presence of background populations when modeling angular momentum evolution. For example, angular momentum lost from tidally synchronized binaries is extracted from the orbital rather than the spin angular momentum reservoir, which results in very different angular momentum evolution properties (see Andronov et al. 2006, for a discussion in the context of blue stragglers).

These large data sets have spurred renewed interest in the usage of stellar rotation as an age indicator, or gyrochronology Barnes (2003). Distinct theoretical scal- 

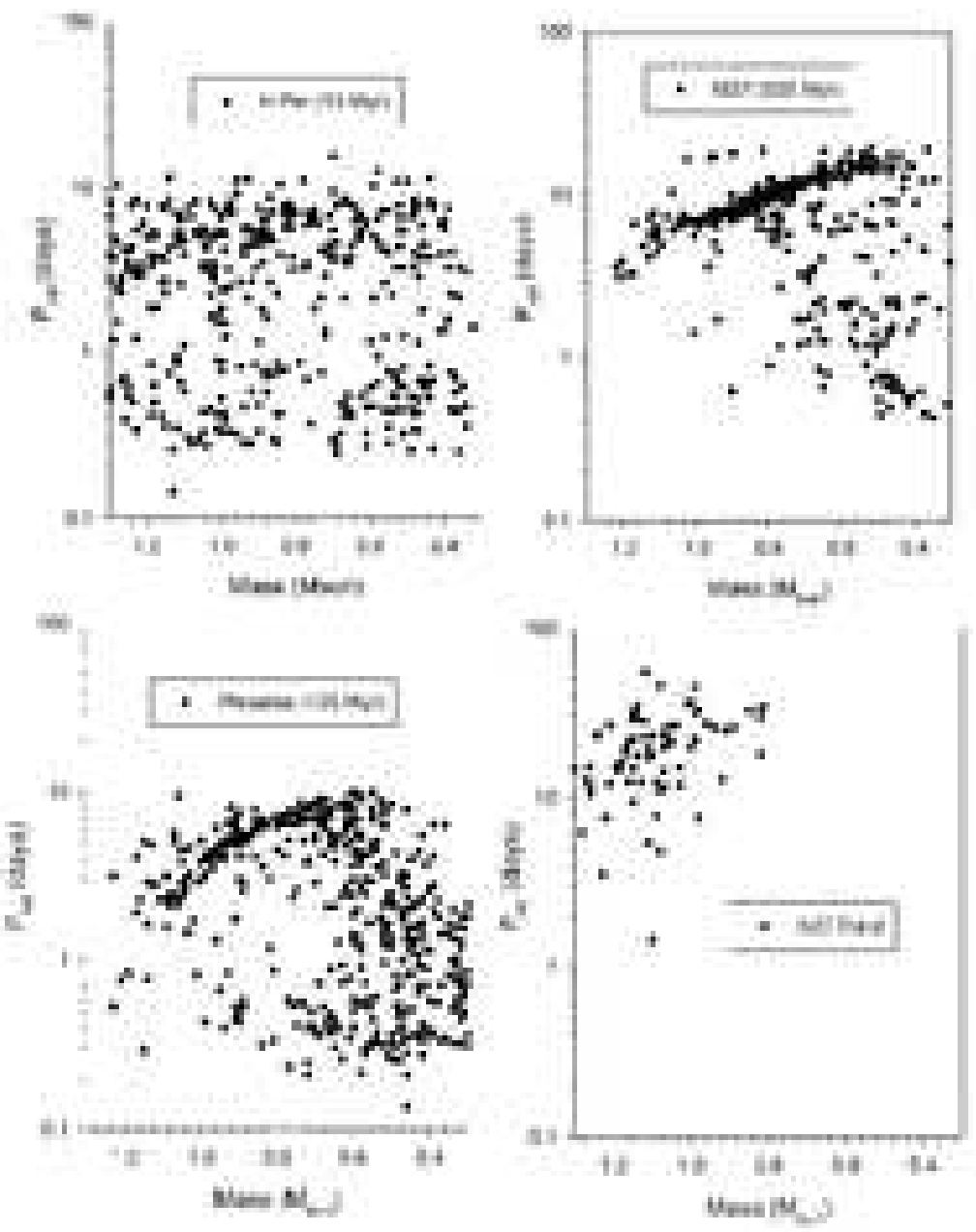

Fig. 3 The observed distribution of rotation rates in stars of different ages. Data for the young protostars in H Per (upper left) is taken from Moraux et al. (2013). The data for the 125 Myr old Pleiades system (lower left) is taken from the reanalysis by Coker \& Pinsonneault (2015, in prep). M37 data is originally from Hartman et al. (2009) as interpolated onto mass by van Saders and Pinsonneault (2013). Data for old field dwarfs is taken from Garcia et al. (2014).

ings and fitting function have been proposed (e.g. Mamaiek and Hillenbrand 2008; Barnes and Kim 2010; Epstein and Pinsonneault 2014, for a discussion). There are significant zero-point shifts between these formulations, which will require resolution when compared with empirical data. There are strict lower bounds to the uncertainties in gyrochronology relations even for an ideal theoretical framework. Epstein and Pinsonneault (2014) identified two fundamental limiting effects for the precision of gyrochronology: 
The intrinsic range of rotation rates at fixed age and the range of surface rotation rates in a given star from latitudinal differential rotation. The former is more important for young and low mass stars, while the latter dominates for older higher mass stars. The net effect is an age uncertainty at the 2 Gyr level for old stars. van Saders and Pinsonneault (2013) extended this to subgiants, and argue for a different gyrochronology relationship for them than for dwarfs. Recently the gyrochronology concept has been adapted to consider magnetic field instead of rotation as a proxy for age: e.g. magnetochronology (Vidotto et al. 2014). It is indeed clear (see next section) that stellar magnetic properties vary over the course of stellar evolution, older stars being less active their younger counterparts (Giampapa 2005; Gregorv et al. 2012).

The theoretical interpretation, numerical models and multi-D simulations allowing us to study and to understand the stellar age-rotation-activity relationship will be discussed in $\S 2$.

\subsection{Stellar Activity \& Magnetism}

The great advantage of comparing the Sun to other stars is to disentangle what is specific to our star from what is generic to solar-like stars. Systematic observations of rotation and magnetic properties of solar-like stars have revealed interesting trends. For instance surface differential rotation is found to increase with $T_{\text {eff }}$; F-star possess a larger latitudinal contrast than K-stars do (Donahue et al. 1996; Barnes et al. 2005; Collier Cameron 2007). Similarly a rotation-activity relationship has been known for quite some time now (Pizzolato et al. 2003). A relation between cycle and rotation periods has also been advocated (Noves et al. 1984b; Böhm-Vitense 2007).

Meaningful information regarding magnetic cycles underlying stellar activity, or alternatively to rule out the existence of magnetic cycles, require long-term stellar activity observations and their analysis. Direct observations of starspots is a relatively new capability and studies of their behavior over timescales long enough to be relevant for stellar dynamos, do not yet exist. Nonetheless, indirect evidence of magnetic activity cycles (or the lack thereof!) can be obtained by different techniques. Our traditional tools for measuring stellar cycles have been synoptic stellar chromospheric activity observations, complemented with more limited stellar coronal X-ray variability data. Asterseismology may prove a useful additional set of data.

The most important contribution in the context of long-term stellar activity observations has been the seminal Mount Wilson Observatory (MWO) Calcium (Ca) $\mathrm{H}+\mathrm{K}$ Project (Wilson 1978b; Noyes et al. 1984b; Baliunas et al. 1985, 1995). The emission in these line cores, expressed as a $\mathrm{Ca} \mathrm{H}+\mathrm{K}$ activity index, is understood to be a measure of the magnetically mediated, non-thermal heating of the chromosphere (provided that the basal thermal flux is properly accounted for, e.g. Schrijver et al. 1989; Cuntz et al. 1999). Thus, the long-term variation of the $\mathrm{Ca} \mathrm{H}+\mathrm{K}$ index can be taken as a proxy for variability in stellar magnetic fields (see the review by Hall 2008). The Lowell Observatory's Solar-Stellar Spectrograph program provides complementary observations of Sun-like stars (Hall et al. 2007; Hall 2008; Hall et al. 2009) adding to the knowledge base of the MWO program. An independent measure of stellar magnetic output is the coronal X-ray flux - which originates in the magnetic heating of stellar coronae and which is even more tightly correlated with magnetic flux (Pevtsov et al. 2003). Although stellar X-ray cycle observations are rare, the amplitude of stellar X-ray flux 
have been observed for multiple stars (Hempelmann et al. 1996; Micela and Marino 2003; Güdel 2004) and this usefully constrains the strength of the dynamo generated magnetic fields. More recent surveys analyzing the activity of solar-like stars are to be found in Wright et al. (2004) and Giampapa et al. (2006). We provide here a brief summary of the important trends and relationships relevant for stellar activity cycles that have been gleaned from these long-term observations.

\subsubsection{General Activity Trends with Rotation}

First we focus on the scaling behavior of magnetic activity with the stellar rotation rate. Stars that are faster rotators have higher magnetic activity amplitudes than slower rotators. They are usually variable, but do not display regular cycles. Stars with intermediate rotation rates have intermediate magnetic activity output, which sometimes display periodic behavior. Stars which rotate relatively slowly, such as the Sun, are more likely to have regular, periodic cycles, but their activity amplitudes are also relatively smaller. The latter category also sometimes display "flat" (i.e., nonvarying) activity with low amplitude, akin to global magnetic minima such as the solar Maunder minimum. We note here that fast rotators also sometimes display a "flat" (non-varying) activity profile albeit with high activity amplitudes- which is not to be confused with global minimum like states.

In general the activity amplitudes increase with rotation rates, but for very fast rotators, the measured amplitudes tend to reach a saturation (Pizzolato et al. 2003; Wright et al. 2011), which in X-rays is of order $\log \left(L_{x} / L_{b o l}\right) \sim-3.2$. For G type stars this saturation is found for rotation rate above $35 \mathrm{kms}^{-1}$, for $\mathrm{K}$ type stars at about $10 \mathrm{kms}^{-1}$ and for $\mathrm{M}$ dwarfs around $3-4 \mathrm{kms}^{-1}$. How stellar magnetic flux scales with rotation rate is important to understand, since it is telling us how the magnetic field generated by dynamo action inside the stars emerges and imprints the stellar surface. Understanding the apparent saturation phenomenon is also important: for example, it could arise if only the filling factor $f$ reaches a maximum (Reiners 2012). Multiperiodic cycles are also sometimes observed, especially in faster rotators (Oláh et al. 2009). For those stars with cyclic magnetic activity, the systematic analysis of stellar data revealed that for solar type stars there is a good correlation between the cycle and rotation periods of the stars and that correlation is even stronger when using the Rossby number $\left(R o=P_{r o t} / \tau\right)$ that takes into account the convection turnover time $\tau$ at the base of the stellar convective envelope (Noves et al. 1984b 1984a); Soon et al. 1993; Baliunas et al. 1996). As the star rotates faster, its cycle period is found to be shorter. This trend is summarized in Figure 4 . More precisely, Noves et al. (1984a) found that $P_{\text {cyc }} \propto P_{\text {rot }}^{n}$, with $n=1.25 \pm 0.5$. Saar and Brandenburg (1999); Saar (2002) have used an extended stellar sample to argue that there are actually two branches when plotting the cycle period vs the rotation period of the stars: the primary (starspot) cycle and the Gleissberg, or grand minima type, modulation of stellar activity. For the active branch they found an exponent $n \sim 0.8$ and for the inactive one they inferred $n \sim 1.15$.

\subsubsection{Maunder Minima Stars:}

Many of the stars (roughly 15\%, see Giampapa et al. 2006), studied in the Mount Wilson surveys, displayed activity that were flat (i.e., non-cyclic) and at or below current solar minimum levels. Similar studies with Kepler data recently found that among 


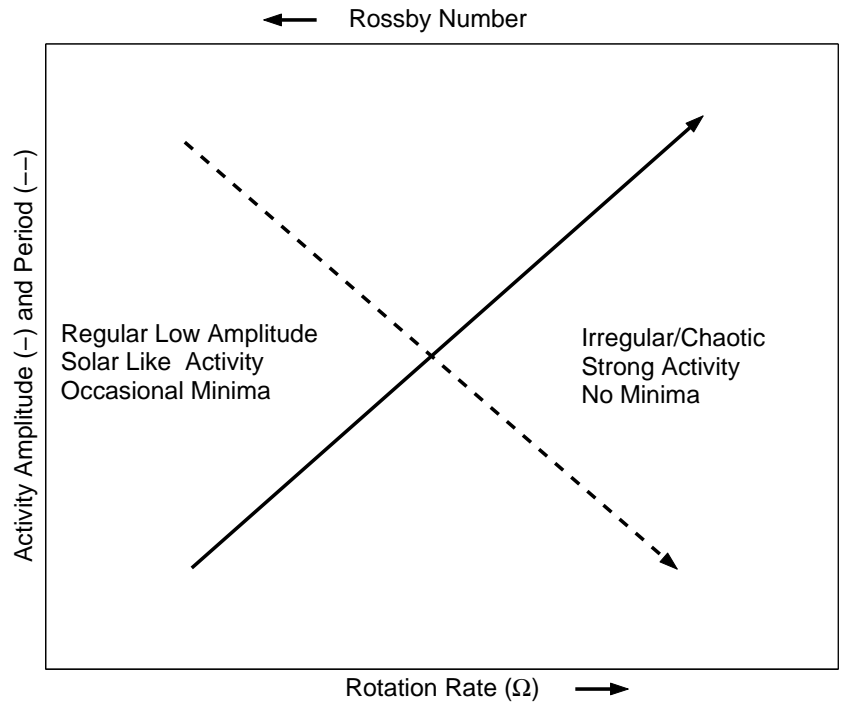

Fig. 4 Stellar activity trends as determined from observations of $\mathrm{Ca} \mathrm{H}+\mathrm{K}$ and X-ray emission from F-G-K stars. The arrows in the axis label show the direction of increase of the rotation rate and Rossby number. In general, magnetic cycle amplitude increases while cycle period decreases with increasing rotation rate (i.e., decreasing rotation period). The critical reader is referred to the original observations cited in the text for the exact scaling laws and other subtleties in the data. From Nandv (2004).

the sampled solar-like seismic stars about $2 \%$ show a flat activity level. One possible interpretation of these results is that these are "Maunder minima" stars where the global magnetic activity has temporarily ceased. Complementary studies of coronal emission also point to the existence of such low-activity stars (Poppenhäger et al. 2009). Baliunas et al. (1995) also provides evidence indicative of a star entering into a Maunder minimum like phase - with its activity variation flattening out accompanied by a reduction in amplitude.

\subsubsection{Starspots, Polar Caps and Stellar Butterfly Diagrams:}

Recent advances in stellar photometry and doppler imaging techniques now allow us to observe starspots and their dynamics. Such observations (reviewed in Berdyugina 2005) show that there is oftentimes a preferred longitude for starspots. Faster rotators tend to have large polar spots which contributes to a strong polar cap, while in general, in very active stars, the spot distribution tends to be more uniform across all latitudes. In some stars, the spots evidently appear at mid-low latitudes and subsequently migrate to higher latitudes, reminiscent of the sunspot migration (although towards low latitude) that generates the solar butterfly diagram. These observations do not span a long period of time and hence detailed studies of stellar butterfly diagrams is still in its infancy. Nevertheless, observations of spot emergence latitudes and migration are expected to provide more stringent constraints in the future for stellar dynamo and flux emergence models. 


\subsubsection{Asteroseismology and Activity}

Ensemble asteroseismology can now add to our developing understanding of stellar activity obtained by other methods, such as spectropolarimetry and CaII H \& K studies.

The first contribution of helio and astro-seismology comes through their ability to provide stellar properties and, in particular, stellar ages for field stars that are otherwise difficult to analyze. Our lack of knowledge of stellar ages has been an important limitation in our understanding of the Mt. Wilson survey data, for example. Many of the solar-like stars showing a flat S-index in the original sample analyzed by Baliunas and Jastrow (1990) -and assumed to be in a similar state to the Maunder minimum in the Sun- were found to be more evolved stars (which would be expected to less active) when accurate parallaxes from the Hipparcos mission were taken into account (Wright 2004). Therefore, accurate stellar properties are essential for proper interpretation of their magnetic indexes (see an extended discussion in the review by Judge and Thompson 2012). But seismology can also be employed to directly study stellar activity. Magnetic fields modify the conditions inside the stars and the properties of the modes are changed accordingly. Seismology can therefore provide complementary information to the one obtained by the above mentioned techniques. Moreover, it can for example provide evidences of the existence of subsurface on-going magnetic cycles even during Maunder minimum-like states (Beer et al. 1998) in which no external manifestation of the internal magnetism can be observed. However, the amplitude of the modes decreases for active stars as observed in the Sun (e.g. Jiménez-Reyes et al. 2003), HD 49933 observed by CoRoT (García et al. 2010), and solar-like stars observed by Kepler (Chaplin et al. 2011a) difficulting their observation.

The first diagnostic or "proxy for magnetic activity" used in helioseismology was the frequency shift of the acoustic modes, i.e., the displacement of the modes towards higher frequencies as the cycle evolves. These frequency shifts - which follow an inverse mode-mass scaling (Libbrecht and Woodard 1990) - are explained to arise from changes in the outer layers of the Sun along the 11-year magnetic cycle (Schwabe cycle) (Goldreich et al. 1991; Balmforth et al. 1996; Dziembowski and Goode 2004, 2005). Therefore, the high-frequency $\mathrm{p}$ modes - having higher upper turning points than the low-frequency p modes - are then more sensitive to the perturbations induced by the magnetic field in the outer part of the Sun. Thus, these high-frequency p modes are observed to have large frequency shifts with the Schwabe cycle, while the low-frequency p modes present little or no variation with it (e.g., Ronan et al. 1994; Chaplin et al. 1998; Gelly et al. 2002; Howe et al. 2008; Jain et al. 2009). A similar behavior has also been found in the F star HD 49933 (Salabert et al. 2011a).

It is now commonly accepted that, apart from the rotational splitting (García et al. 2008; Broomhall et al. 2012), all the rest of the solar low-degree p-modes properties change with time (e.g. Chaplin et al. 2000; Jiménez-Reves et al. 2003, 2007; Salabert et al. 2011b; García et al. 2013) in a time scale of 11 years following the corresponding surface Schwabe cycle. Another shorter timescale has been recently confirmed using helioseismology. Indeed, the quasi-periodic modulations of 1 to 2 years observed during the last twenty years in the Sun (e.g. Benevolenskava 1995; Mursula et al. 2003) has then been measured (Fletcher et al. 2010) in the frequency shifts of low-degree modes measured by the Global Oscillation at Low Frequencies (GOLF Gabriel et al. 1995)) instrument on board the Solar and Heliospheric Observatory (SoHO Domingo et al.|1995), as well as with the Birmingham Solar Oscillation Network (BiSON Chaplin et al. 1996). Although the origin of this modulation is not well understood yet, the latest results priv- 
iledge the hypothesis that the origin of the perturbation is located in the subsurface layers (Simoniello et al. 2013).

The continuous photometric monitoring of hundreds of thousands of stars by CoRoT and Kepler provide long baseline stellar variability samples of unprecedented scale and precision. Their duration is also substantial, comprising a period of more than 4 years in the case of Kepler; this is long enough to begin to see the effect of activity cycles. The first ensemble analysis Kepler light curve varibility was done by Basri et al. (2010) and Basri et al. (2011). However, because the Kepler pipeline was not designed to measure long-period stellar variability they used raw data from only a 90 day quarter, which introduced important limitations in their analysis. Basri et al. (2011) found that the amplitude of variability was in general larger for stars that were clearly periodic than for those that were not. As expected, the largest group of non-periodic variables were giant stars. To improve on the Kepler pipeline, which was designed for transit detection and not stellar variability, several groups developed their own correction software (e.g. García et al. 2011; McQuillan et al. 2014; Garcia et al. 2014). With these new analysis tools it was possible to detect longer-than-a-month rotation periods as well as to study the magnetic variability of the stars.

Based on broadband photometric variability, a new proxy for stellar magnetic activity has been developed (García et al. 2010; Chaplin et al. 2011a; Mathur et al. 2014b). The time evolution of the variance of the signal appears to be a good indicator of the magnetic activity cycle. It has been successfully tested on the Sun using VIRGO/SPM data recorded during the last 16.5 years (see for more details García et al. 2013). Using the same methodology, Mathur et al. (2014a) studied a subset of 22 Kepler F stars with rotation periods of less than 12 days for which p-mode oscillations were reported. Because of the relation between the rotation period of the star and the period of the cycle (e.g. Böhm-Vitense 2007; Jouve et al. 2010), they were expecting to see in some stars cycle-like variations such as the one observed by Metcalfe et al. (2010) in the F8V star $i$ Horlogii. That star exhibited a magnetic cycle period of 1.6 years for a rotation period of 8.5 days. Mathur et al. (2014a) found that only a small fraction of stars showed a regular cycle-like behavior (see Fig (5) and there were no clear trends between activity and rotation in the F-star sample. A more systematic study still needs to be done to confirm or refute the existence of a general relationship between stellar cycle period and stellar rotation period for these hot stars. For solar analogs recent analysis of Kepler data have confirmed an rotation-age relationship do Nascimento et al. 2014).

\section{Global Models of Stellar Structure, Rotation, and Magnetism}

In order to understand, characterize and describe the physical processes at the origin of stellar convection, rotation and magnetism, with the Sun being an archetype of solarlike stars, we must develop a self-consistent, ideally nonlinear, description of their dynamics. Depending on the time scale of interest and given the current computing resources such model can be either 1-D, 2-D or 3-D. In the following section we briefly summarize the recent progress made in modeling the multi-scale dynamics of stars and their success at explaining the observed properties discussed in $\S 1$. 


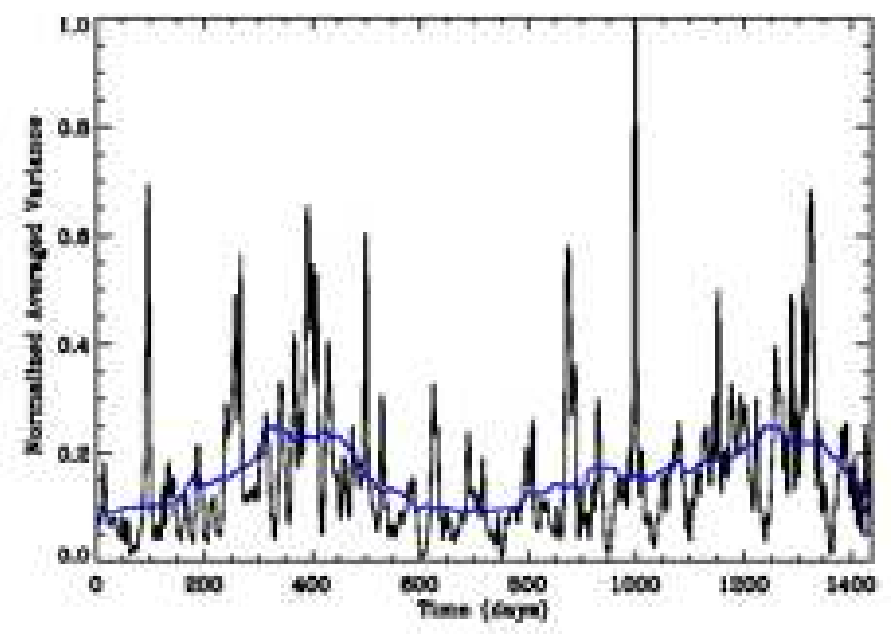

Fig. 5 Evolution of the normalized averaged variance of the Kepler F star KIC 12009504 for which p-mode oscillations have been measured (in black) and smoothed by a boxcar function (160 days wide) in blue. A regular cycle of around 800 days is observed (see Mathur et al. 2014a, for further details).

\subsection{1-D stellar evolution and core-envelope coupling}

The angular momentum evolution of a star - its rotational history from its birth until its death has profound consequences for its structure, evolution, and dynamics. Rotationally induced mixing for low mass stars can induce mild mixing that alters surface abundances (see Pinsonneault 1997 for a review), while for massive stars, which rotate much more rapidly, the impact of mixing is far more dramatic (see Maeder and Mevnet 2012, for a recent review). Stellar rotation is also intimately connected with the origin and generation of stellar magnetic fields, and the two together shape wind outflows from stars. Hence it is fundamental to be able to characterize it. The overall picture of angular momentum evolution was discussed in section 1.3. Here we focus on modeling.

Structural evolution in stars will naturally generate internal angular velocity gradients, and in some evolutionary stages these shears can be large. Mass loss from the surface will also carry angular momentum away from the star, providing another powerful driver for radial differential rotation. In convective regions the interaction of rotation, magnetism, and convection will enforce an angular momentum distribution on a convective overturn timescale, much shorter than the natural nuclear evolution timescale. The response of radiatively stable regions is more complex, both because of our limited knowledge of the physics and because of limited empirical constraints on the relevant time scales. Internal redistribution by hydrodynamic and magnetohydrodynamic physical process can both be important, and different classes of models testing different prescriptions have been employed. 
Solar-like stars on the main sequence evolves on long time scales; the solar main sequence lifetime is of the order 10 Gyr. Computing such long temporal (secular) evolution with multi-D codes is impractical. One must rely on 1-D stellar evolution models to describe the structure, chemical and rotational evolution of stars. We refer to the recent review by (Basu et al. 2014) for a detailed discussion of 1-D stellar evolution codes, their success and difficulty at reproducing the solar internal structure, the influence of chemical composition, and how such helioseismically calibrated 1-D solar models have been extended to compute up-to-date 1-D stellar models. Here we focus on internal angular momentum transport and its consequences for the rotation history of stars. To this end, let's apply the concept of angular momentum exchange and conservation to a star like our Sun.

A useful framework that has been extensively employed in the literature is the two zone model of MacGregor and Brenner (1991). In this model, the solar-like star is treated as a sphere divided into two zones: an inner stably stratified core and a turbulent convective envelope both independently rotating around an axis aligned with the $\hat{\mathbf{e}}_{z}$ direction, where $\hat{\mathbf{e}}_{z}$ is the unit vector along the $\mathrm{z}$ axis of a 3 -D $(x, y, z)$ cartesian system (see Fig 6 left panel). Each possess their own moment of inertia $I$ and angular velocity $\Omega$; both structural evolution and magnetized winds can generate differential rotation. Their angular momentum is respectively $J_{\text {core }}=I_{\text {core }} \Omega_{\text {core }}$ and $J_{\text {env }}=I_{\text {env }} \Omega_{\text {env }}$. Let's now find the exact quantity of angular momentum exchange $\Delta J$ between the two zones require to have them rotate uniformly at the rotation rate $\Omega$. The initial state is: $J_{\text {init }}^{c}=J_{\text {core }}$ and $J_{\text {init }}^{e}=J_{\text {env }}$. Applying total angular momentum conservation e.g. $J_{\text {init }}^{c}+J_{\text {init }}^{e}=J_{\text {final }}^{c}+J_{\text {final }}^{e}$, implies that the final state is: $J_{\text {final }}^{c}=J_{\text {core }}-\Delta J=I_{\text {core }} \Omega$ and $J_{\text {final }}^{e}=J_{\mathrm{env}}+\Delta J=I_{\mathrm{env}} \Omega$. A simple substitution yields:

$$
\Delta J=\frac{I_{\text {env }} J_{\text {core }}-I_{\text {core }} J_{\text {env }}}{I_{\text {core }}+I_{\text {env }}} .
$$

If an external torque, such as the one coming from a stellar wind $\tau_{\text {wind }}$ is applied to the star, the angular momentum evolution of the convective envelope and radiative interior can be expressed as (MacGregor and Brenner 1991):

$$
\begin{aligned}
\frac{d J_{\text {core }}}{d t} & =-\frac{\Delta J}{t_{\mathrm{c}}} \\
\frac{d J_{r m e n v}}{d t} & =\frac{\Delta J}{t_{\mathrm{c}}}-\frac{J_{\mathrm{env}}}{t_{\mathrm{w}}},
\end{aligned}
$$

with $t_{\mathrm{c}}$ the coupling time scale between the two zones due to the combined action of turbulence, waves, magnetic fields and viscous stresses and $t_{\mathrm{w}}$ the wind braking timescale. These formula further assumes that the convective envelope transmits instantaneously the applied surface torque to the base of the convective envelope.

Such models have been used to explore the relevant coupling time scale between the convective envelope and the radiative interior in solar-like stars over the course of their evolution (MacGregor and Brenner 1991; Keppens et al. 1995; Krishnamurthi et al. 1997; Allain 1998) and (for recent developments see Denissenkov et al. 2010; Bouvier 2013; Gallet and Bouvier 2013; Oglethorpe and Garaud 2013; Zhang and Penev 2014). It is found that a time scale of tens to hundreds of Myr can explain the core-envelope coupling in young open cluster stars (see Figure 6).

One can identify key angular momentum transport processes which are expected to act inside a stellar convection zone or radiative interior: Reynolds and Maxwell 

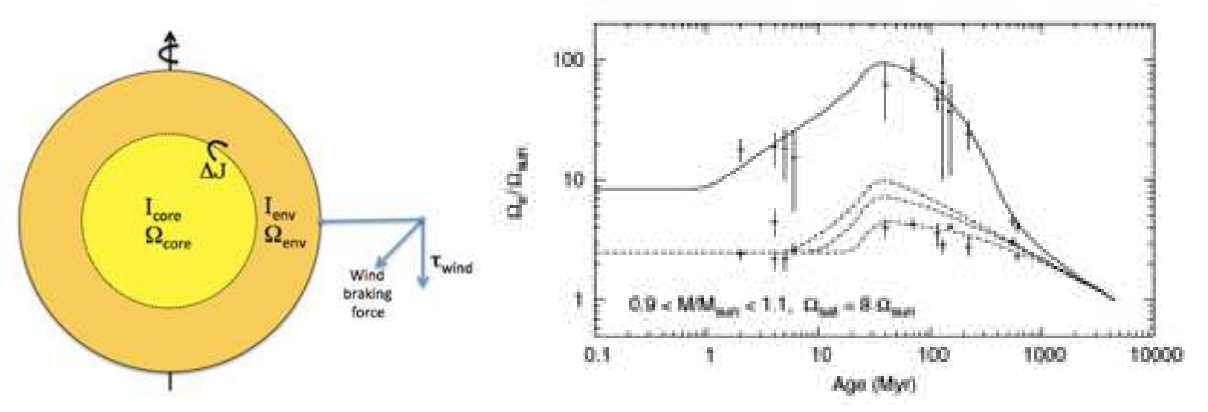

Fig. 6 Left: The two layers model of a stars, showing the exchange of angular momentum $\Delta J$ between a core and an envelope rotating at different rates and the braking action of a stellar wind. Right: Evolutionnary track for two layer models showing how the coupling time scale can explain slow and fast rotators (from Denissenkov et al. (2010))

stresses, meridional circulation, viscous processes (usually negligible), large scale magnetic torque, gravity waves (see Gilman et al. 1989; Zahn 1992; Pinsonneault 1997; Mathis and Zahn 2004, 2005; Denissenkov et al. 2010; Mathis 2011; Brun et al. 2011; Reiners and Mohanty 2012; Mathis 2013b; Gallet and Bouvier 2013; van Saders and Pinsonneault 2013, and references therein). The amplitude and role of each process varies as a function of time. For example, it is known that young stars are more active than old ones, so internal Maxwell stresses are expected to play a less significant role later in the star's evolution on the main sequence and likewise for the stellar wind torque. Of course when the star undergoes a large structural change due to its secular evolution (as for instance when it goes up the red giant branch) the relative importance of different processes must be re-evaluated.

Kawaler (1988) proposed a general analytical formulation of the applied torque that has been widely used. He adopted the (Mestel 1968) framework and adopted parameters tuned to reproduce Skumanich's law (Skumanich 1972) for the time dependence of surface rotation. Many other studies have since improved upon Kawaler's model by taking into account better observational information or theoretical improvements in order to assess how the magnetic torque applied by a stellar wind depends on stellar parameters and dynamics (rotation, dynamo); see for example the Rossby-scaled saturation threshold adopted by Krishnamurthi et al. (1997), which has been extensively employed subsequently. There has been much recent activity in this area, with new frameworks being proposed by (Cranmer and Saar 2011; Matt et al. 2012; Reiners and Mohanty 2012; van Saders and Pinsonneault 2013; Brown 2014; Matt et al. 2014).

Self-consistent solar models including angular momentum transport from hydrodynamic mechanisms were first generated in the 1980s (Endal and Sofia 1981; Pinsonneault et al. 1989). Meridional circulation, hydrodynamic instabilities and gravity waves are the primary mixing and angular momentum transport agents, so these models play an important role in rotational mixing studies (Mathis 2013a; Brun and Mathis 2014). Such models are sometimes coupled with approximations of magnetic effects, such as the Taylor-Spruit mechanism (Spruit 2002). This approach has been used to model massive rotating stars over secular time scales (Palacios et al. 2003; Marques et al. 2013; Meibom et al. 2013) and to generate grids of stellar models used for isochrones 
and chemical evolution studies (Ekström et al. 2012; Georgy et al. 2013; Lagarde et al. 2012). Some attempts have also been made to model in 2-D the secular evolution of internal angular momentum by considering the role of large scale magnetic field in the radiative interior (see Charbonneau and MacGregor 1993; Ruediger and Kitchatinov 1996; Spada et al. 2010, and references therein). However such models do not solve for dynamo action as we will now discuss in the next section.

\subsection{2-D mean field stellar dynamo models}

On time scales of centuries to millenia it is possible to model in 2-D the magnetohydrodynamics of stars using the mean field dynamo framework by parameterizing or neglecting some of the detailed description of convective motions. This allows one to explore the underlying physics and a wide range of stellar parameter space which we describe below.

\subsubsection{The Theoretical Framework}

Stellar magnetic cycles involve the generation and conversion of two components of the Sun's magnetic field - the toroidal component and the poloidal component. Under the assumption of axisymmetry, the stellar magnetic field and plasma flow can be expressed as

$$
\begin{aligned}
\mathbf{B} & =B_{\phi} \mathbf{e}_{\phi}+\nabla \times\left(A \mathbf{e}_{\phi}\right) \\
\mathbf{v} & =r \sin (\theta) \Omega \hat{\mathbf{e}}_{\phi}+\mathbf{v}_{p}
\end{aligned}
$$

In this axisymmetric decomposition, the first term on the R.H.S. of Eqn. 9 is the toroidal component and the second term the poloidal component of the magnetic field (of which $A$ is the vector potential). The first term on the R.H.S. of Eqn. 10 signifies the $\phi$-component of the velocity (i.e., angular velocity $\Omega$ ), while $\mathbf{v} p$ denotes the meridional $(r-\theta)$ component of the velocity.

Our understanding of solar and stellar cycles is based primarily on 2-D kinematic $\alpha \Omega$ dynamo models and full MHD simulations (cf. §2.2, Brun et al. (2013) (also published as chapter 5 of the ISSI book (Thompson et al.)) and Weiss 1994; Charbonneau 2010), most of which have been constructed in the solar context. Here we briefly review this understanding. The toroidal component of the magnetic field is produced by stretching of an initial poloidal field by the differential rotation inside stars (the dynamo $\Omega$-effect) and is thought to be stored in a layer stable to convection near the base of the convection zone. Strong toroidal flux tubes rise up due to magnetic buoyancy and erupt as magnetic (star) spots. The toroidal flux must be recycled to poloidal flux for the dynamo cycle to perpetuate and this conversion mechanism (traditionally termed as the dynamo $\alpha$-effect) could be achieved by various means. The two most popular and widely studied mechanisms are the mean-field $\alpha$-effect driven by helical turbulence (Parker 1955) and the decay and dispersal of tilted bipolar active regions - i.e., bipolar sunspot or starspot pairs (Babcock 1961; Leighton 1969), hereby the BL mechanism. It is thought that strong toroidal flux tubes, believed to be necessary to produce sunspots, would quench the mean-field $\alpha$-effect, if there is no segregation of the poloidal and toroidal generation process and thus the BL mechanism must be 
the dominant mechanism for poloidal field generation in the Sun; however, this debate is far from over and we will return to this later. We display in Figure 7 a typical representation of a BL like dynamo model.
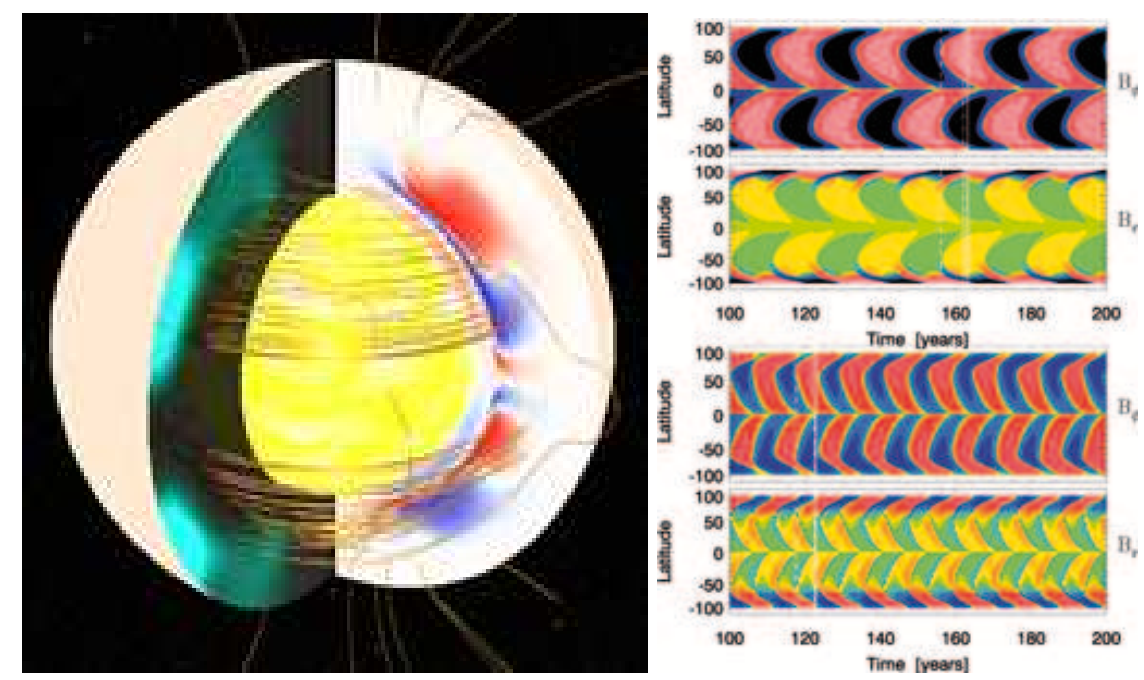

Fig. 7 Left: a 2.5D kinematic dynamo simulation depicting a snapshot of the toroidal (right meridional plane) and poloidal field (left meridional place) configurations at a phase close to solar minimum. The simulation shows that the toroidal component of the field is generated and stored deep in the convection zone, from where it buoyantly erupts to form sunspots. The poloidal field is created in the near-surface layers in this particular dynamo model of the Babcock-Leighton type (Nandv et al. 2011). 3-D rendering of field lines (magenta) show the expected wound-up field structure in the solar interior. Right: Butterfly diagram for 2 models representative of pumping dominated dynamo, rotating at respectively $0.7 \Omega_{\odot}$ and $3.0 \Omega \odot$. We note the equatorward branch, the correct phase relationship between the toroidal and poloidal component of field (shown as vertical lines) and the shorter rotation period for the case rotating at three time the solar rate (Do Cao and Brun 2011).

In the kinematic approach, the plasma velocity is prescribed and the magnetic field evolution equation is solved under the assumption of no direct magnetic feedback on the flows. The magnetic induction equation is given by

$$
\frac{\partial \mathbf{B}}{\partial t}=\nabla \times(\mathbf{v} \times \mathbf{B}-\eta \nabla \times \mathbf{B}) .
$$

On substituting Eqn. 9 and Eqn. 10 in the induction equation and separating out the toroidal and poloidal magnetic field components we get the evolution equations for the poloidal and toroidal components, respectively:

$$
\begin{aligned}
& \frac{\partial A}{\partial t}+\frac{1}{s}\left[\mathbf{v}_{p} \cdot \nabla(s A)\right]=\eta\left(\nabla^{2}-\frac{1}{s^{2}}\right) A+S_{\alpha}, \\
& \frac{\partial B_{\phi}}{\partial t}+s\left[\mathbf{v}_{p} \cdot \nabla\left(\frac{B_{\phi}}{s}\right)\right]+\left(\nabla \cdot \mathbf{v}_{p}\right) B_{\phi}=\eta\left(\nabla^{2}-\frac{1}{s^{2}}\right) B_{\phi}+s\left(\left[\nabla \times\left(A \hat{\mathbf{e}}_{\phi}\right)\right] \cdot \nabla \Omega\right) \\
& +\frac{1}{s} \frac{\partial\left(s B_{\phi}{ }^{\natural}\right)}{\partial r} \frac{\partial \eta}{\partial r} \text {. }
\end{aligned}
$$


Here $s=r \sin (\theta)$ and $\eta$ is the turbulent diffusion coefficient. The source term for the poloidal field $S_{\alpha}$ could denote either the mean-field $\alpha$-effect (in which case $S_{\alpha}=\alpha B_{\phi}$ ) or the BL source due to the buoyant eruption and flux dispersal of tilted active regions. The flow in the meridional plane $\mathbf{v}_{p}$ represents meridional circulation $\left(\mathbf{v}_{p}=\mathbf{v}_{m}\right.$, where $\mathbf{v}_{m}$ denotes the meridional circulation), and in the presence of turbulent pumping (say $\mathbf{v}_{\gamma}$ ) it represents the combined effect of both (i.e., $\mathbf{v}_{p}=\mathbf{v}_{m}+\mathbf{v}_{\gamma}$ ). Appropriate prescriptions for the flows, turbulent diffusion and nature and profile of the poloidal field source along with the boundary conditions, completely describes the kinematic dynamo system defined in Eqn. 12 and Eqn. 13.

In BL flux transport dynamo models, the cycle period $P_{c y c}$ is found to depend strongly on the meridional circulation amplitude $v_{0}$ and its profile and less on the rotation rate $\Omega_{0}$ or the amplitude of the surface source term $s_{0}$ Dikpati and Charbonneau 1999; Jouve and Brun 2007):

$$
P_{c y c} \propto \Omega_{0}^{0.05} S_{0}^{0.07} v_{0}^{-0.83}
$$

As will be seen in the next section, the meridional circulation is found to decrease with the rotation rate as $v_{0} \propto \Omega_{0}^{-0.45}$. This is not intuitive as one could expect the meridional circulation velocity to increases with the rotation rate. A careful study of the $\phi$ component of the vorticity equation shows that the longitudinal vorticity actually decreases with rotation rate as more and more kinetic energy is being transferred to longitudinal motions at the expense of meridional motions. The fact that in recent 3-D simulations (Ballot et al. 2007; Brown et al. 2008; Matt et al. 2011; Augustson et al. 2012 ), the meridional circulation is found to weaken as the models is rotated faster implies that standard advection dominated flux transport dynamo models yield the opposite dependency with rotation than the one observed, e.g. activity cycles are found to be longer for faster rotating stars (Jouve et al. 2010). This fact alone requires us to revise our current dynamo paradigm for solar-like stars. One way is to short-circuit the advection path by, for instance, adding more cells in latitude or increasing the radial diffusion as was done in (Jouve et al. 2010; Hazra et al. 2014); another is to introduce magnetic turbulent pumping (Mason et al. 2008; Guerrero and de Gouveia Dal Pino 2008; Do Cao and Brun 2011). These large-scale magnetic flux transport processes, e.g. meridional circulation, turbulent diffusion and turbulent (or topological) pumping of magnetic flux are extremely important for dynamo operation because they link the spatially segregated source layers of the dynamo, specifically coupling the near-surface layers to the deep convection zone. The competition between these processes influences the overall behavior of the dynamo significantly (Jouve and Brun 2007; Yeates et al. 2008; Guerrero and de Gouveia Dal Pino 2008; Karak and Nandv 2012; Do Cao and Brun 2011). Meridional circulation is also thought to critically influence the nature of magnetic butterfly diagrams, including their structure, overlap between cycles and cycle periods (Charbonneau and Dikpati 2000; Nandy and Choudhuri 2002; Jouve and Brun 2007; Nandy et al. 2011; Hazra et al. 2014) and also impacts scaling relationships in stellar activity (Nandv 2004; Jouve et al. 2010). However, the exact profile of the meridional flow remains unconstrained in stars. For the Sun, evidence for more than one meridional flow cell are being reported (Haber et al. 2002; Mitra-Kraev and Thompson 2007; Zhao et al. 2013) and there is serious debate about its relative role vis-a-vis other flux transport processes. 
For instance new stellar dynamo models including magnetic pumping Do Cao and Brun (2011) yield the following dependency for the cycle period $P_{c y c}$ :

$$
P_{c y c} \propto v_{0}^{-0.40} \gamma_{r 0}^{-0.30} \gamma_{\theta 0}^{-0.15}
$$

where $\gamma_{r 0}$ and $\gamma_{\theta 0}$ represent the amplitude of the radial and latitudinal turbulent pumping terms. We see that the cycle period dependency on the meridional circulation is reduced and that if we want to recover the observational trends, in particular the shorter cycle period (again, we assume that $v_{0} \propto \Omega^{-0.45}$ ), and assuming that $\gamma_{r} / \gamma_{\theta}$ remains constant, the pumping effect should roughly scale as $\Omega_{0}^{2}$. Such a scaling may be too extreme and only systematic 3 -D numerical simulations will tell us if this is the case or not. Nevertheless, pumping dominated stellar dynamos are a plausible solution to explain stellar observations.

\subsubsection{Stellar Activity and Magnetism: Confronting Mean Field Dynamo Models with Observations}

The general trends in stellar activity observations, in particular the rotation, cycle amplitude, and cycle period relationship is succinctly expressed when confronted with the stellar Rossby number, $R_{o}=P_{\Omega} / \tau_{c}$, the ratio of the rotation period, $P_{\Omega}$ to the convective turn-over-time of the star (Durnev and Latour 1978; Baliunas et al. 1996; Montesinos et al. 2001; Jouve et al. 2010). This number can be calculated using a combination of observations with some theoretical ideas about the nature of stellar convection zones, assuming that the convective turn-over-time is $\tau_{c}=L / V=L^{2} / \eta$, where $L$ is a characteristic length scale, $V$ a typical convective speed and $\eta$ the magnetic diffusivity. The latter equality is valid if the magnetic Reynolds number $R_{m}=V L / \eta$ is taken to be of order unity, an assumption that depends on the properties of stellar convection zones and on the convection zone depth. It can be interpreted as characterizing turbulent mixing of magnetic fields in stellar interiors, while the rotation period plays a role in determining differential rotation. It is well known (both theoretically and observationally) that the differential rotation in the convective envelope of solar-type stars is directly connected to the star's rotation rate $\Omega_{0}$ (Donahue et al. 1996; Barnes et al. 2005; Ballot et al. 2007; Brown et al. 2008; Küker and Rüdiger 2008; Matt et al. 2011; Augustson et al. 2012). However, the exact scaling $n_{r}$ (i.e. $\Delta \Omega \propto \Omega_{0}^{n_{r}}$ ) is still a matter of debate among the observers and theoreticians, being sensitive to the observational techniques used and to the modelling approach (see $\S 2.2$ ). Note that the $\alpha$-effect used in mean field theory is related to helical turbulence and represents a measure or parameterization of the electromotive mean force (emf) (e.g. Moffatt 1978; Pouquet et al. 1976). It is thus also linked to the rotation rate of the star and the amount of kinetic helicity present in its convective envelope. However in Babcock-Leighton flux transport dynamo models the classical $\alpha$-effect is replaced by a surface term linked to the tilt of the active regions with respect to the east-west direction (the so-called Joy's law, e.g. Hale et al. 1919; Babcock 1961; Kosovichev and Stenflo 2008; Dasi-Espuig et al. 2010). This tilt is thought to be due to the action of the Coriolis force during the rise of the toroidal structures that emerge as active regions (D'Silva and Choudhuri 1993). Recent 3-D simulations in spherical shells (Jouve and Brun 2009; Fan 2009; Weber et al. 2011; Pinto and Brun 2013; Jouve et al. 2013; Weber et al. 2013) seem to indicate that this is not the only effect responsible for the observed tilt and that the twist and arching of the toroidal structures as well as the continuous action of the surface convection 
during the emergence influence the resulting tilt. The Rossby number, overall, characterizes stellar convection zones - the zone where the dynamo mechanism generates stellar magnetic fields. Not surprisingly, the Rossby number is simply related through scaling arguments to the dynamo number, $D \sim 1 / R_{O}{ }^{2}$. Thus the Rossby number can be utilized to relate stellar observations to the dynamo mechanism (see e.g., Noves et al. 1984b 1984a; Tobias 1998; Montesinos et al. 2001; Nandv 2004).

On can also implement a non linear feed back via the large scale Lorentz force (also called the Malkus-Proctor effect; Malkus and Proctor (1975)) on the dynamo field generation. This has the advantage to yield a saturation (quenching) process based on the actual dynamics of the models (Tobias 1997; Moss and Brooke 2000; Bushby 2006, and references therein). These models demonstrate that for low magnetic Prandtl number (e.g. $P_{m}=\nu / \eta$ ), such that the viscous time scale is much longer than the magnetic diffusion time scale, the dynamo is irregular, with grand minimumlike behavior and switching of parity between symmetric and antisymmetric dynamo families as observed in the Sun (DeRosa et al. 2012).

From the observational perspective, analysis shows that stars with low Rossby numbers typically exhibit strong activity amplitudes and have irregular cycles, while those stars with relatively higher Rossby numbers tend to have lower activity levels, are more likely to host magnetic cycles and are more likely to be found in grand minima like phases. Since lower Rossby number indicates a higher, underlying dynamo number, and vice-versa, this result is consistent with dynamo theory. Stars which host very efficient dynamos (having high $D$ ) are expected to have high activity levels; the non-linear nature of the dynamo mechanism, in conjunction with a high dynamo number also generates irregular cycles because the magnetic feedback is likely stronger. On the other hand, stars with low or moderate dynamo numbers are relatively less active, and are more likely to host regular cyclic behavior with occasional Maunder-like states such as the Sun. The consistency of stellar observations with the theoretically expected behaviour of a MHD dynamo mechanism leaves little doubt that internal dynamos are the ultimate source of stellar activity.

A closer look at the activity period versus rotation rate relationship yields more stringent constraints on the nature of stellar dynamos. In particular, it is seen that the periods of stellar magnetic cycles decrease with increasing rotation rate (i.e., decreasing rotation period $P_{\Omega}$ ). As discussed in $\S 2.1$, there is an inter-dependance between dynamo action, magnetic activity levels, rotation and stellar evolution via the torque that magnetized thermally driven stellar winds apply on solar-like stars. The mass dependance of this torque is subject to intense studies (see Bouvier 2013, for a recent discussion). It is understood that solar dynamo models that rely on the BL mechanism for poloidal field generation are critically dependent on flux transport processes such as meridional circulation which couples the source regions for the toroidal and poloidal components of the magnetic field. Such BL dynamo models have been successful in reproducing various features of the solar cycle. Based on such a BL dynamo model, Nandy (2004) has shown that the stellar activity amplitude and period versus rotation rate relationship can only be reproduced if both the rotational shear and meridional flow speed scales positively with rotation rate. While full MHD numerical simulations show that the former holds, they also indicate that meridional circulation speed does not increase with rotate rate (Augustson et al. 2012). Do Cao and Brun (2011) point out in a recent study that the inclusion of turbulent pumping, with the speed of the former increasing with rotation rate, is necessary to recover the stellar scaling laws in the light of these new developments in full MHD simulations. Dubé and Charbonneau 
(2013) have also computed 2-D kinematic dynamo using ingredients (such as the alpha effect) deduced from 3-D simulations. They show that essential ingredients of the complex 3-D dynamics (such as for instance the cycle period or the sense of propagation of the dynamo wave) can be recovered via such techniques. 3-D kinematic dynamo models or convective B-L models have also recently been developed (Chan et al. 2007; Miesch and Brown 2012; Miesch and Dikpati 2014).

Hence confronting stellar activity observations with diverse simulation techniques is very useful and allows the community to test new ideas and concepts.

We now discuss in more details nonlinear, 3-D MHD simulations of solar convection zone and magnetism.

\subsection{3-D Global Models of Stellar Convection and Dynamo Action}

With the advent of massively parallel computers it is now possible to attack the difficult problem of stellar dynamics and magnetism with direct 3-D nonlinear numerical simulations. As is frequently the case with multidimensional MHD numerical simulations, two approaches are possible: local high resolution simulations that describe the small scale turbulent motions and local dynamo (Cattaneo 1999; Vögler and Schüssler 2007) and global ones that take into account the correct geometry and the existence of large scale flows in rotating convective zones Glatzmaier (1985a, 1985b); Brun et al. (2004); Miesch and Toomre (2009). Given the broadness of the topic, here we present the progress made in modeling stellar convection and dynamos with the global approach. These simulations allow us to describe turbulent, rotating and magnetized convection either in a full sphere, a shell or a wedge-like geometry (cf. the recent book by Charbonneau 2013a). Contrary to the kinematic mean field stellar dynamo approach discussed in the previous sections, 3-D simulations use the full set of MHD equations (we refer to these recent references for simpler 2-D models of stellar differential rotation assuming a parametrization of turbulent convection Küker and Rüdiger 2008; Hotta and Yokoyama 2011; Kitchatinov and Olemskoy 2012). These equations couple classical fluid mechanic equations to the induction equation and as such do not prescribe the velocity field but compute it self consistently (c.f. Brun et al. 2004; Miesch 2005). This is the main advantage of the global full MHD approach: the influence of rotation and magnetic field on turbulence and convection is computed not parametrized.

Over the last three decades several groups have developed stellar convection and dynamo models in spherical geometry following the pioneering work of P. Gilman and G. Glatzmaier in the early-mid 80's (Gilman and Miller 1981; Glatzmaier and Gilman 1982; Gilman 1983; Glatzmaier 1985a 1985b). Prominent examples of modern global or wedge-like parallel codes that have in recent years published original studies on stellar convection and dynamo include:

\footnotetext{
- the Anelastic Spherical Harmonics (ASH) code: Miesch et al. (2000); Brun and Toomre (2002); Ballot et al. (2007); Miesch et al. (2008); Browning (2008); Bessolaz and Brun (2011); Augustson et al. (2012) on surface convection and zonal flows and Brun (2004); Brun et al. (2004); Browning et al. (2006); Brown et al. (2008, 2010, 2011); Brun et al. (2011); Miesch and Brown (2012); Nelson et al. (2013); Augustson et al. (2013); Nelson et al. (2014) on solar-like star dynamo action, cycles and magnetic wreaths,
} 
- the Eulag MHD code: Ghizaru et al. (2010); Racine et al. (2011); Charbonneau (2013b) on solar and stellar dynamo and cycles,

- the PENCIL code: Warnecke et al. (2013); Käpylä et al. (2013); Guerrero et al. (2013) on stellar convection, dynamos and cycles,

- the Magics code: Christensen and Aubert (2006); Christensen et al. (2009); Christensen (2010); Gastine et al. (2013, 2014) on convection and dynamos

- K. Chan's code Cai et al. (2011) on core convection

Other codes have been mostly used to model the geodynamo with some applications to the dynamo in $\mathrm{M}$ dwarfs.

- Glatzmaier's code: adapted to model Earth's dynamo Glatzmaier and Roberts (1996) or giant planets dynamics Glatzmaier et al. (2009),

- EBE code: Chan et al. (2007),

- Leeds code Hollerbach and Jones (1993); Sreenivasan and Jones (2006) ; dynamo review by Jones (2011)

- PARODY code: Goudard and Dormy (2008); Morin et al. (2011); Schrinner et al. (2012, 2014) on geo and stellar dynamos and Aubert et al. (2007) on the role of thermal wind in setting mean large scale flows and Aubert et al. (2008) on dynamo

- Busse and Simitev's code: Grote and Busse (2001); Simitev and Busse (2009) on dynamo and bistability

- Aurnou and Heimpel's code: Heimpel et al. (2005); Aurnou et al. (2007) on dynamo in convective envelopes

- Several research groups in Japan: Takehiro and Havashi (1999); Yoshida and Kagevama (2004); Kimura et al. (2011) on convection or zonal flows or Kagevama and Sato (1997); Nishikawa and Kusano (2008); Masada et al. (2013) on dynamo, solar convection and magnetism, the most recent studies making use of a novel "ying-yang" grid to map a spherical shell (Kagevama and Sato 2004).

Many of the codes used in the geophysics community were originally developed to model the geodynamo. They therefore rely on the Boussinesq approximation, which is not appropriate for highly stratified and compressible convective envelope as found in solarlike stars. Modelers have been converting these codes to be able to deal with stellar-like stratified flow by using the anelastic approximation (Gough 1969; Glatzmaier 1984; Vasil et al. 2013) and validating their codes with a recent international benchmark (Jones et al. 2011).

Given the intrinsic complexity of computing nonlinear convection and dynamo simulations and the large diversity of stars to model, we first briefly describe purely hydrodynamical models (see also chapter 3 in the ISSI book (Thompson et al.)). Such models reveal the fundamental ingredients at the origin of large scale mean flow, such as differential rotation and meridional circulation in rotating global convection models. We then follow up by describing the origin of magnetic fields (either cyclic or irregular) through nonlinear dynamo action and the feedback of such fields on the flows - making the link with the 2-D models discussed earlier when necessary.

\subsubsection{Convection and Large Scale Flows}

Convection is present in stars of all masses on the main sequence. It takes either the form of a convective core in massive ones (greater than about $1.3 M_{\odot}$ ) or of a convective envelope of varying thickness for lows mass stars (from early $\mathrm{F}$ down to 
about M3), with stars less massive than about $0.3 M_{\odot}$ being fully convective. Here we will limit our discussion to solar-like stars possessing a deep convective envelope. For a discussion on two other categories (e.g. high mass and very low mass stars) interested readers may refer to (e.g. Browning et al. 2004; Brun et al. 2005; Dobler et al. 2006; Brown et al. 2008; Christensen et al. 2009; Featherstone et al. 2009; Augustson et al. 2011; Cai et al. 2011; Morin et al. 2011; Schrinner et al. 2012, and references therein).
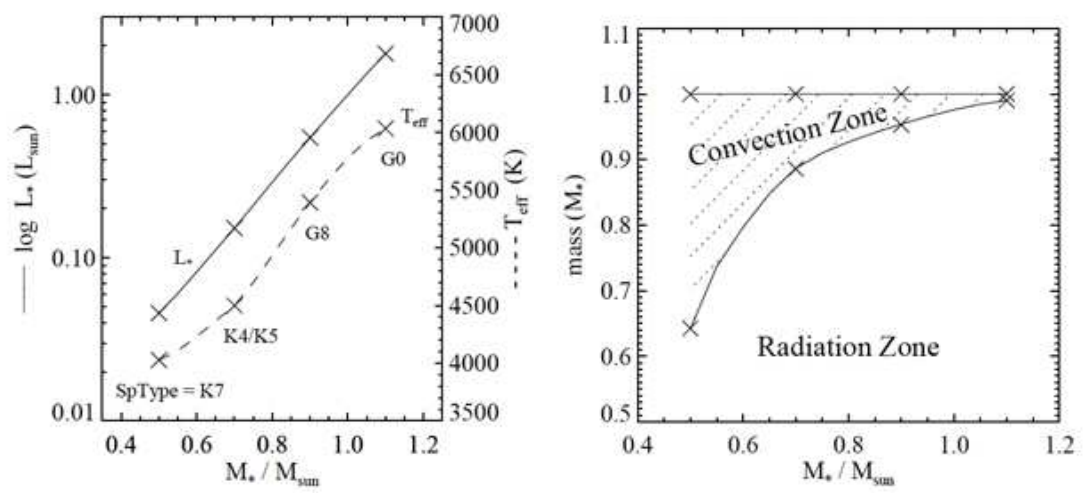

Fig. 8 Stellar luminosity, effective temperature and mass contained in the convective envelope vs spectral type in solar like stars with the solar metallicity (computed with the CESAM code Morel 1997)
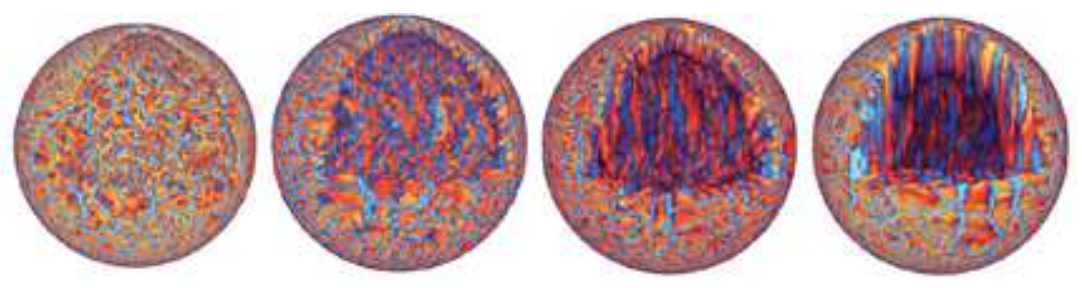

Fig. 9 3-D rendering of turbulent surface convection in solar-like stars with varying convection depths (Bessolaz and Brun 2011). The radial velocity is shown with cold downflows in blue tones.

Convection plays a key role in transporting the star's heat by having strong correlations between vertical motions and temperature fluctuations. It is a fundamentally turbulent process in stars as the Rayleigh number ( $R a$, e.g. the ratio between buoyancy driving and dissipation process) is huge, of order $10^{18}$ in the Sun. This leads to a large range of scales from possibly giant cells on the order of the thickness of the convective layer (e..g $200 \mathrm{Mm}$ in the Sun) down to granulation size $(1 \mathrm{Mm})$, with intermediate scales such as supergranulation $(20-50 \mathrm{Mm})$ well delineated by the surface magnetic network (Rieutord and Rincon 2010). As the luminosity (energy output) of the star changes so does the amplitude of the convective flow. A simple scaling argument based on mixing length treatment (MLT) gives $v_{r} \propto\left(L_{*} /\left(\rho_{c z} R_{*}^{2}\right)\right)^{1 / 3}$, with $L_{*}$ 
and $R_{*}$ respectively the star's luminosity and radius and $\rho_{c z}$ the averaged density of the convective envelope (Kippenhahn et al. 2013). Since the luminosity varies by at least 2 orders of magnitude across the spectral type we are considering (i.e early $\mathrm{F}$ to early M) $\left(L_{*} \propto M_{*}^{4}\right)$, the stellar radius only by a few $\left(R_{*} \propto M_{*}^{0.9}\right)$, it is expected that the vigor of the convective flows do likewise modulo the effect of density. As can been seen in Figure 8, 1-D stellar structure models of solar-like stars indicate that the averaged mean density changes significantly in the convective envelope of these stars, in such a way that more massive stars have on average a lower mean density than low mass ones (Matt et al. 2011). This is easily understood as the base of the envelope moves up towards the surface with the thinning of the convective envelope. This yields a large amplitude difference between $\mathrm{F}$ and $\mathrm{K}$ stars for their characteristic convection velocity. This difference in velocity amplitude is recovered in global numerical simulations of stellar convection. We illustrate an example of such 3-D turbulent convective simulations in Figure 9 where we clearly see the complex network of downflows surrounding broad upflows.

The turbulent motions within the convective envelopes of rotating stars experience the effect of the Coriolis force to a degree that depends on their fluid Rossby number $R o=\omega / 2 \Omega_{*}$, here defined as the ratio between turbulent and the so-called planetary vorticity (Pedlosky 1982). We note that several definitions of the Rossby number exist in the community (stellar Rossby based on the ratio between convective turnover time and stellar rotation period; convective Rossby $\sqrt{R a / T a P r}$; local Rossby number), but it can be shown that they depend almost linearly on one another (see for example Kim and Demarque 1996; Landin et al. 2010; Matt et al. 2014). We here choose to use the fluid Rossby $R o$ because a clear transition of behavior occurs at $R o \sim 1$ with the differential rotation becoming prograde or retrograde. Indeed if the stars rotate fast enough such that the large scale motions (larger than the Rossby radius of deformation) feel the continuous action of the Coriolis force as they rise and sink then the redistribution of angular momentum is such that a non uniform rotation profile is established in the convective envelope.

In figure 10 we display 4 realizations of the longitudinally averaged differential rotation and meridional circulation computed with the ASH code (Matt et al. 2014, in preparation). These profiles have been obtained by changing either the stellar mass (i.e aspect ratio and luminosity) or the reference frame rotation rate. We note several trends: As the rotation rate is increased, the differential rotation becomes more and more aligned with the rotation axis (along $\hat{e}_{z}$ ) and likewise the meridional circulation posses more and more cells both in latitude and radius. The 1.1 solar mass case rotating at one time solar rate exhibits an anti-solar differential rotation, with slow equator and fast pole. It is indeed the only case with a Rossby number greater than 1 as seen in many recent studies (Bessolaz and Brun 2011; Matt et al. 2011; Guerrero et al. 2013; Gastine et al. 2014; Käpvlä et al. 2014). We thus see that the transition between prograde and retrograde differential rotation will not occur at the same rotation rate $(v \sin i)$ for each stellar spectral type. We also note the strong shear layer at the base of the convective domain (e.g. the tachocline Spiegel and Zahn 1992) that naturally develops in each modeled stars with a profile closer to the solar case for all the cases with a Rossby number $R o \sim 0.6$.

These profiles can be understood by considering the transport of angular momentum in a spherical convective shell. When performing this analysis as in Brun and Toomre 2002; Brown et al. 2008; Augustson et al. 2012), we find that the Reynolds stresses are at the origin of the prograde equator for models with a low Rossby number. Through 

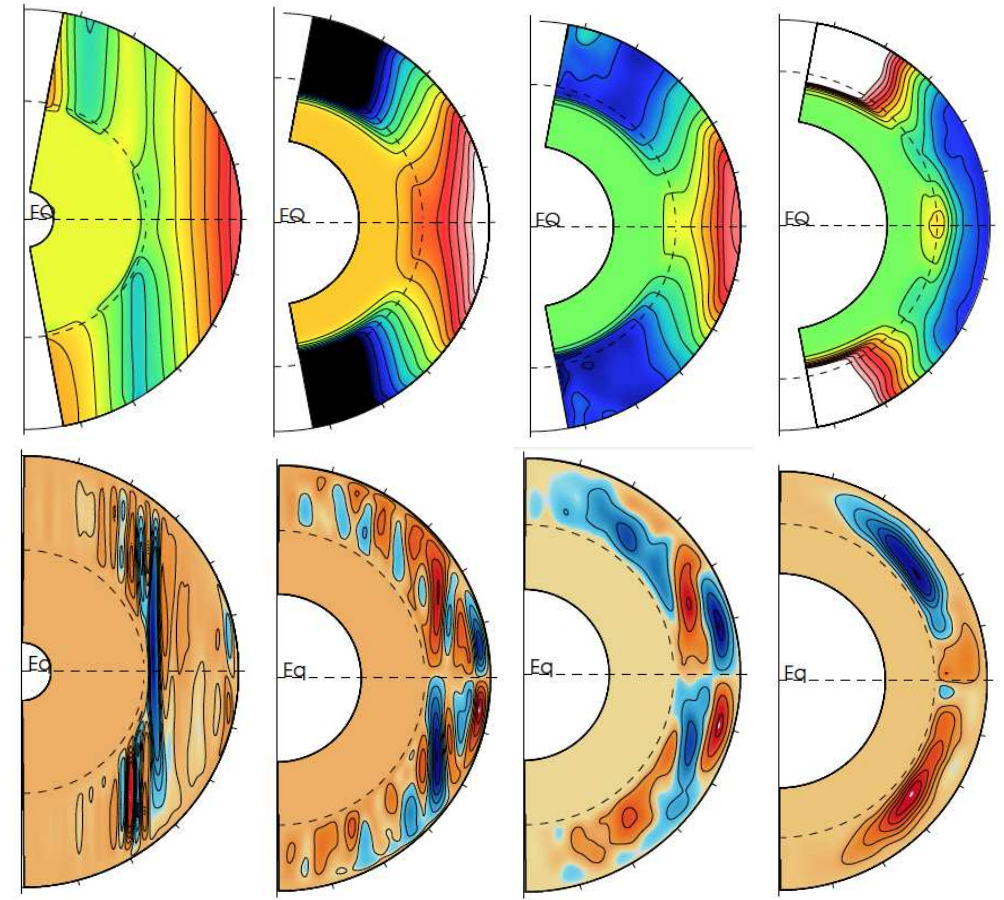

Fig. 10 Differential rotation and meridional circulation realized in 3-D models of solar-like convective envelope coupled nonlinearly (i.e both zones back react on one another) to a stably stratified interior (Matt et al. 2014, in preparation)). 3 masses (0.5, 0.9 and 1.1) $M_{\odot}$ and 3 rotation rates (solar, 3 and 5 time solar) are being shown. Starting from the left in each row: color contours showing $\Omega(r, \theta)$ and the meridional streamfunction for a $0.5 M_{\odot}$ star rotating at $5 \Omega_{\odot}, 0.9 M_{\odot}$ star rotating at 3 and $1 \Omega_{\odot}$ and $1.1 M_{\odot}$ star rotating at $\Omega_{\odot}$. Prograde rotation is shown in red/white colors and retrograde rotation in dark/blue. Clockwise meridional flows in red.

gyroscopic pumping the meridional circulation transport angular momentum to counterbalance the torque applied by the Reynolds stresses (from which we have subtracted the contribution from viscous effect). We refer to (Zahn 1992; Mathis and Zahn 2004; McIntyre 2007; Garaud and Bodenheimer 2010; Brun et al. 2011; Miesch and Hindman 2011) for a more thorough discussion of the role of the meridional circulation in reaching angular momentum balance in rotating stars. The orientation of the iso-contours of $\Omega$ from being cylindrical to being more conical (solar-like) at mid latitude is due to the effect of an efficient thermal wind associated with latitudinal entropy variations (with relatively hotter poles and cooler equator) Miesch et al. (2006). These variations are enhanced further by the presence of a tachocline (Brun et al. 2011).

One important aspect of performing 3-D numerical simulations is that one can obtain general trends on the variations of the main physical processes acting in convective envelope with various parameters such as rotation rate, luminosity, aspect ratio. We focus on scaling laws for the differential rotation and meridional circulation by synthesizing the recently published 3 -D stellar convection models computed with the ASH code. The models range from 0.5 up to 1.3 times the solar mass and from 1 to 20 times the solar rotation rate (Brown et al. 2008; Matt et al. 2011; Augustson et al. 2012). In 

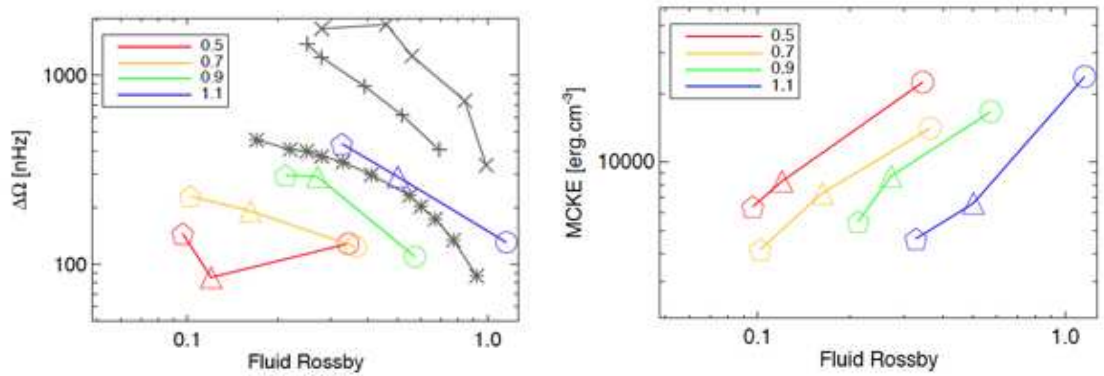

Fig. 11 Differential rotation $\Delta \Omega$ as a function of Rossby number for stellar masses ranging from 0.5 up to $1.3 M_{\odot} .1 M_{\odot}$ cases from (Brown et al. 2008), 1.2 and $1.3 M_{\odot}$ cases coming from (Augustson et al. 2012)). Kinetic energy of the axisymmetric meridional circulation as a function of the Rossby number for models ranging from 0.5 to $1.1 M_{\odot}$, from (Matt et al. 2014, in preparation).

Figure 11 we show how the latitudinal differential rotation contrast varies as a function of the Rossby number. There is a clear trend for $\Delta \Omega$ to rise with increasing rotation rate. This is in qualitative agreement with direct and indirect observational data that imply larger absolute shear rates in rapid rotators to produce stronger magnetic fields and enhanced angular momentum loss relative to slow rotators. The actual slope seems a bit larger than observed by Collier Cameron (2007) but not so when compared with the work by Donahue et al. (1996). Saar (2011) found that the presence of binaries in the published samples, which can be distinct from single stars because of tidal effects have led to an underestimate of the dependency of $\Delta \Omega$ on rotation rate. Clearly more observational data are required to constrain better the exponent $n$ in the equation $\Delta \Omega \propto \Omega_{*}^{n}$. We also clearly see that more massive stars possess larger $\Delta \Omega$ for a given Rossby number. This is in reasonable agreement with the observations published in Barnes et al. (2005). Although, given the paucity of data and the rather large error bars, the exact exponent is uncertain. Data from the Kepler mission and in the (future) PLATO mission will be a major asset in this context (Garcia et al. 2014; Rauer et al. $2013)$.

Another interesting trait is that the meridional circulation is found to decrease in strength as the stars are made to rotate faster. Rapid rotation causes more kinetic energy to go into toroidal motions, which can be anticipated by looking at the equation for gyroscopic pumping (Landin et al. 2010; Augustson et al. 2012). This has a very important consequence: Flux-transport dynamo models as discussed in the previous section can't be easily extended to other stellar spectral type to explain the dependence of their cycle period with their rotation period. Indeed flux-transport models of the advective type depends directly on the meridional circulation amplitude to set the cycle period. Observations indicate that the cycle period should decrease with increasing rotation rate. However, given that the meridional flow is found to weaken, this can only result in an increase of the activity cycle as was shown by Jouve et al. (2010). One needs either to take into account multi-cellular flows as observed also in 3-D simulations (Jouve et al. 2010) or take into account turbulent pumping (Do Cao and Brun 2011). 
2.3.2 Nonlinear Dynamo Effect, Magnetic Activity, and Cycles

The observed magnetic activity shows the existence and period of cycles seem to depend on stellar spectral type and rotation rate. An obvious trend is that old (older than the age of the Hyades cluster $\sim 800 \mathrm{Myr}$ ), slowly rotating solar-like stars are less active than their younger counterparts. We have also discussed in the previous section that convection and large scale flows change with spectral type. It is certainly not surprising that the associated dynamo action and magnetic activity do so as well.

Several groups have recently published extensive parameter studies of dynamo action in rotating convective spherical shells of various thickness and degree of stratifications. The key results can be summarized as follow: 3-D numerical simulations of dynamo action in solar-like stars have revealed a large range of behavior, from steady dynamo, to irregular and cyclic ones (Brun et al. 2004; Brown et al. 2010, 2011; Racine et al. 2011; Gastine et al. 2012; Schrinner et al. 2012; Augustson et al. 2013; Käpvlä et al. 2013; Nelson et al. 2013; Schrinner et al. 2014, and references therein).

For low mass (M-type) stars, it is seen that a bistability similar to that found for the geodynamo is possible. Observations by Morin et al. (2010) seems to indicate that a strong dipolar state and a multipolar state may coexist for stars having similar stellar parameters. Dynamo models show that around a local Rossby number of 0.1 a weak (multipolar) and strong (dipolar) dynamo branch may coexist Christensen and Aubert (2006); Morin et al. (2011). Such bistable states are found in a given range of parameters (low stratification, high $\mathrm{Pm}$ ) and may not actually exist in real stars. It may be the case that these stars are observed in a different phase (low vs high) of their activity cycle. Other similarity between the geodynamo and low mass stellar dynamos also exist such as the scaling of the magnetic energy with the available heat flux Christensen et al. 2009). Such scaling tends to break down for higher mass stars for which the tachocline may play a significant role.

Others 3-D simulations of F, G and K stars show that for high rotation rates, large scale magnetic wreaths (see Figure 13 left panel) are obtained without requiring the presence of a tachocline Brown et al. (2010, 2011). We show on Figure12, four butterfly diagrams (time-latitude plots of the azimuthally averaged toroidal magnetic field near the base of the $\mathrm{CZ}$ ) realized in such simulations for of a solar-like star rotating at three times the solar rate Nelson et al. (2013). We remark that as the model is made more turbulent, the steady magnetic wreaths become more time dependent and can lead to cyclic activity (bottom right panel).

In such stars, along with the degree of turbulence, rotation plays an important role in determining the global properties of their magnetism. This is due to a shift in the balance of forces driving the flow between the advection, Coriolis and Lorentz terms. As the rotation rate increases the Lorentz force tends to balance the Coriolis force yielding larger magnetic energy in superequipartion with the kinetic energy of the flow (a direct consequence of a magnetostrophic state; c.f. strong scaling below) as in the Earth's iron core. The Elsasser number $\Lambda=B^{2} / 4 \pi \bar{\rho}_{c z} \eta \Omega_{0}$, with $\bar{\rho}_{c z}$ mean density in the convective envelope, $\eta$ magnetic diffusivity, $\Omega_{0}$ stellar rotation rate, $B$ a characteristic magnetic field of the $\mathrm{CZ}$, is useful to discuss this balance of terms in the Navier-Stokes (N.V.) equation. Depending on the amplitude of this number and on the balance assumed in the Navier-Stokes equation, various scaling of the magnetic field amplitude can be expected (Fauve and Pétrélis 2007; Christensen 2010): 

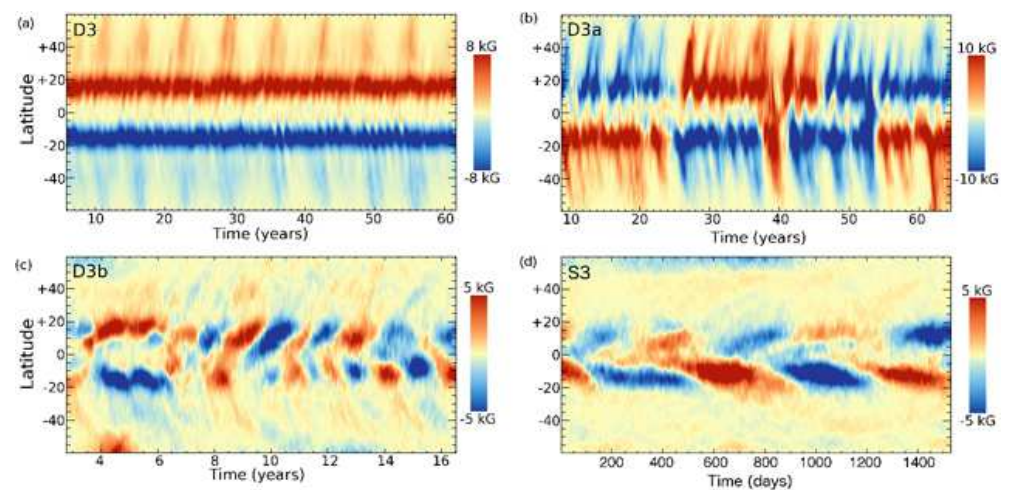

Fig. 12 Magnetic wreaths yielding in turn steady (case D3; top left), irregular (case D3a; top right) and quasi cyclic (cases D3b \& S3; bottom left and right) magnetic butterfly diagrams Nelson et al. (2013). Red tones correspond to positive toroidal polarity.
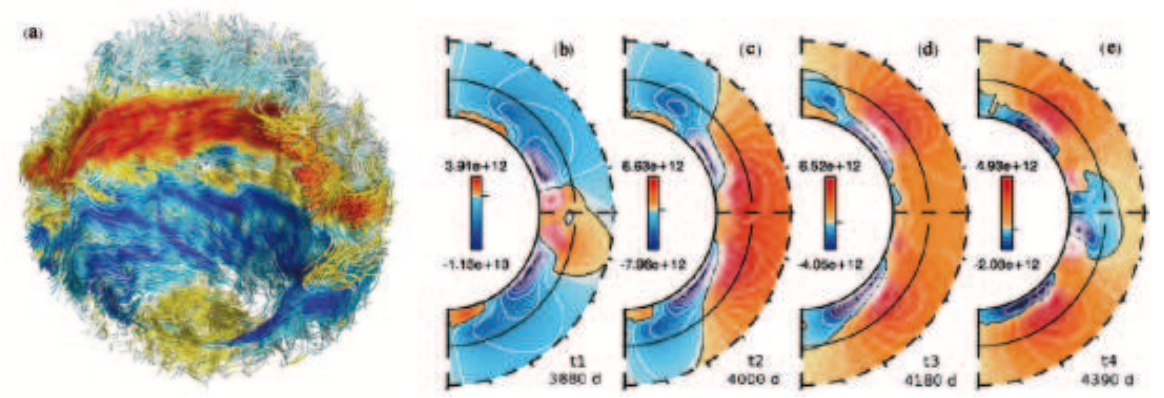

Fig. 13 Magnetic wreaths shown by 3-D rendering of magnetic field lines (Brown et al. 2010). Temporal sequence of meridional cuts showing the mean poloidal field realized in case D5 of Brown et al. (2011) which was extrpolated using potentiel field approximation to about 1.5 stellar radius. Note the global polarity field reversal of the poloidal field.

- To an order of magnitude, an estimate of the equilibrium magnetic field (assuming ideal gas law) can easily be obtained: $B_{e q} \sim \sqrt{8 \pi P_{g a s}} \sim \sqrt{\bar{\rho}_{c z}}$, since $T_{\text {eff }}$ varies by a factor 2 to 3 between early $\mathrm{F}$ and late $\mathrm{K}$ stars, whereas $\bar{\rho}_{c z}$ varies by more than a factor of 100 (Matt et al. (2011)).

If one assumes for the sake of simplicity that the magnetic Reynolds number $R_{m}=$ $v d / \eta \sim 1$ such that a characteristic velocity is given by $v \sim \eta / d$, the balance of terms in Navier-Stokes Equation is then given by:

- Laminar (weak) scaling: Lorentz $\sim$ viscous diffusion $\Rightarrow B_{w e a k}^{2} \sim \bar{\rho}_{c z} \nu v / d \sim \bar{\rho}_{c z} \nu \eta / d^{2}$

- Turbulent (equipartition) scaling: Lorentz $\sim$ advection

$$
\Rightarrow B_{\text {turb }}^{2} \sim \bar{\rho}_{c z} v^{2} \sim \bar{\rho}_{c z} \eta^{2} / d^{2} \Leftrightarrow\left|B_{\text {weak }}\right| \sim\left|B_{\text {turb }}\right| P_{m}^{1 / 2}
$$

- Magnetostrophic (strong) scaling (e.g. Elsasser nb $\Lambda \sim 1$ ): Lorentz $\sim$ Coriolis

$$
\Rightarrow B_{\text {strong }}^{2} \sim \bar{\rho}_{c z} \Omega_{0} \eta
$$

1 note however that such choice of characteristic velocity scale is subject to caution in stellar turbulent convective envelopes that usually possess very large $R m$ 
with $v, d$ are characteristic velocity and length scales, $P_{m}=\nu / \eta$ the magnetic Prandtl number. Of course there is an upper limit to the magnitude of the magnetic energy ultimately set by the amount of energy (likely the star's outward energy flux) that can be made available to the dynamo process (Schubert et al. 1996; Christensen et al. 2009). We recall here that dynamo action does not exist for all fluid motions due to its intrinsic 3-D character (Moffatt 1978).

So a possible scenario for stellar dynamos based on heuristic scaling arguments could be the following: Stars rotating at moderate rate (such that their Elsasser number is small), have a level of magnetic energy (or averaged global field strength) that are less than or of the order of the equipartition field given by either the weak or turbulent scalings described above. As stars rotate faster and get closer to a magnetostrophic state with an Elssasser number of order 1 or larger, the formation of large and intense magnetic wreaths starts. The magnetic field becomes more and more dominated by its toroidal component and the magnetic energy becomes larger and larger going above the equipartition value set by the turbulent scaling as it now follows the strong scaling which depends linearly on the rotation rate. The consequence may be the following. As the magnetic energy (or field amplitude) becomes large, the associated Lorentz force starts back reacting strongly on the mean flow. The first consequence is what can be called an " $\Omega$-quenching", e.g. the differential rotation reduces in strength and an almost solid body rotation state in the convective zone (envelope or core) is established (Brun 2004; Brun et al. 2005). Using mean-field classification, one may then hypothesize that the stellar dynamo transitions from an $\alpha-\Omega$ or $\alpha^{2}-\Omega$ to being an $\alpha^{2}$ dynamo, i.e. helical turbulence is solely responsible for field generation and maintenance, the large scale shear now plays a more marginal role. At that stage what remains of the magnetic wreaths is still unclear, more work must be done. As the rotation is made even faster, quenching of the $\alpha$ effect, due to the large scale magnetic field being more and more intense, occurs naturally as now the Lorentz force back-reacts directly on the helical convective motions and not solely on the large-scale mean flow. Following this discussion, one question naturally crops up which requires detailed investigations: How are these diverse set of plausible dynamo scaling relationships (involving magnetic activity amplitudes) related to stellar activity trends and can the latter impose more stringent constraints on solar and stellar dynamo processes?

As we have seen in section $\S 1.6$, the activity of solar-like stars can be assessed by observing their X-ray luminosity $L_{x}$. Using such proxy it has been shown that magnetic activity increases with rotation rate and that above a given rotation rate (that depends on the stellar spectral type but is around $R_{o s} \sim 0.1$ ), stellar magnetic activity first saturates (Pizzolato et al. 2003; Wright et al. 2011) and then possibly "over-saturates" for extremely fast rotation rate such as the ones found in young solar-like T-Tauri stars (Feigelson and Montmerle 1999). One can thus simply seek to characterize how the saturation of the dynamo generated field can be linked to the saturation of solar-like star X-ray emissivity. Such a link is not as straightforward to deduce as one could anticipate. Indeed one must also assess how the filling factor $f$ of the magnetic field on the star's surface evolves with stellar parameters not just the field strength. As we have noted the field amplitude does not easily saturate since the Lorentz force can achieve a dynamical balance with various terms whose importance and amplitude vary with stellar parameters. One may conjecture that the first saturation of the X-ray luminosity is due to $\Omega$-quenching and to the physical limitation of the spot coverage (e.g., filling factor $f$ ) on the stellar surface and that the second "over-saturation" may be due to " $\alpha$-quenching", so of the actual field strength (see Gondoin 2012 for 
an alternative explanation). Currently no global-scale 3-D nonlinear MHD dynamos actually include spot formation and emergence. Global flux emergence simulations have been computed to assess the role of the Coriolis force, Hoop stresses, convective motions, large scale mean flows and background magnetic fields on the evolution and morphology of simplified active regions by varying the properties of idealized global flux ropes (Fan 2009; Jouve and Brun 2009; Weber et al. 2011; Isık et al. 2011; Jouve et al. 2013; Pinto and Brun 2013; Weber et al. 2013) but until very recently, none had selfconsistently dealt with the formation of sunspots in a cyclic convective dynamo. So in order to be able to physically characterize the saturation of $B$ and $f$ independently, spot-dynamos - wherein dynamos self consistently produce rising omega-loops - must be developed and the parameter space explored systematically.

There has been recent progress in this direction using improved numerical treatment in 3-D simulations of the diffusivities (via dynamical Smagorinsky, slope limited diffusion or implicit large eddy simulation (ILES)). Authors of these studies have been able to lower the diffusivity level low enough to yield more time dependent dynamo solutions possessing both cyclic behavior, regular butterfly diagram and torsional oscillation-like behavior (Käpylä et al. 2011, 2012, 2013; Dubé and Charbonneau 2013; Smolarkiewicz and Charbonneau 2013; Beaudoin et al. 2013; Augustson et al. 2013). Some have even obtained for the first time buoyant magnetic wreaths (Nelson et al. 2011, 2013). In these recent simulations, magnetic wreath-like structures become turbulent and intermittent enough, that many intense bundles of fields reaching $50 \mathrm{kG}$ start to form $\Omega$-loop like structures with statistical properties for rising loops that qualitatively agree with observations (Nelson et al. 2014). We believe that such simulations are the progenitors of future more realistic spot-dynamos that will allow us to characterize the link between dynamo action, flux emergence and large scale magnetic topology as a function of stellar parameters.

Finally also note that the torque applied by the magnetized wind (see discussion in $\S 1.5$ and 2.1) depends mostly on the low order fields and that the field topology is expected to change as a function of stellar cycle and overall rotation rate (see Pinto et al. 2011; Reville et al. 2014). Hence assessing the large-scale magnetic topology of stellar magnetic fields and how it changes as a function of stellar parameters is very important as it has a direct bearing on stellar evolution.

\section{Future Directions}

The characterization of the many facets of stellar dynamics (convection, rotation, magnetism) has made tremendous progress over the last 20 years. We have moved away from a quasi-static view of stars to a time dependent, turbulent and magnetic description of these complex objects. Asteroseismology has significantly contributed to this progress, along with spectropolarimetry and ever improving numerical simulations and models of stellar convection and dynamo action. Still much remains to be done. Many of the assumptions have been deduced by extrapolating our (incomplete) understanding of our star, the Sun, to other stars. The continuous back and forth comparison between the Sun and other stars has been most productive and will continue being so with many projects relying both on ground-based telescopes and space-born missions in the planning. The launch of ESA's M3 Cosmic Vision PLATO in 2024 (Rauer et al. 2013) will continue the rich tradition of data collection initiated by its predecessors Corot and Kepler, helping refine our view of stellar dynamics and magnetism. PLATO 
has a field of view of more than 2200 square degrees and will observe two different fields for two years. It will combine huge statistics (about 300,000 stars) with high temporal resolution and length of observations to properly extract internal and surface stellar rotations as well as signatures of the existence of stellar cycles. Because one of the PLATO fields will include the Kepler field, long-term magnetic variability (of the order of decades) will be potentially unveiled. Fields of shorter observing times of six months (covering in total half of the sky) will add around a million new stars for which oscillations will be measured. This large amount of high-quality seismic data will be a treasure trove for potential new discoveries and will put new constraints on the theories of stellar activity. In the more immediate future, in 2017, the launch of NASA's new planet hunting mission TESS is planned. Although this mission is much smaller in scope than Kepler, TESS will observe all stars in the sky down to magnitude 12.5. Apart from the polar regions of the sky (observed for six months), other fields where the density of stars is higher - will be observed for around 30 days. TESS will therefore contribute significantly to stellar rotation period studies, but it is by design focused on shorter timescale variability studies than PLATO.

Furthermore, other recently launched space missions have already or will soon provide asterosesimic measurements. Apart from the Canadian mission MOST, whose typical 3 weeks observations per target are not necessarily suited for the type of studies we have discussed here, the BRITE constellation of six nano-satellites (http://www.brite-constellation.at) and Kepler's K2 missions are also contributing to the generation new constraints. The latter will be particularly important because it provides the ability to employ the new tools of asteroseismology in well-studied star clusters, such as M67, the Pleiades, and the Hyades. K2 will also sample a variety of stellar populations in the Galaxy, important for our understanding of stellar population effects.

In addition to the space-borne instruments, the Danish-led ground-based Stellar Observations Network Group (SONG, Grundahl et al. (2014)) will soon provide very high-quality spectroscopic asterosesismic data of distant stars. Asterosesismic data observed in velocity posses a much higher signal to noise ratio compared to the photomoetric data obtained in space, but for much less stars though. Two of the planned eight observing sites, distributed all over the Earth, are already funded, and either in construction (China) or already in operation (Teneriffe). The very high-quality data expected from SONG will allow us to obtain, for example, information from the very outer stellar layers with unprecedented accuracy, information which is much needed to improve our knowledge about the still ill-understood surface-effects (see Section 1.2) and the details of the physical mechanisms that drive solar-like oscillations.

The detailed analysis of the legacy star catalog observed by Kepler, the exciting and newly planned asteroseismic observation campaigns, the improvements in computation power and, last but not the least, our own improved understanding of the physical processes in stellar interior will not only bring a wealth of new discoveries, but will also bring stellar physics to a new level of sophistication.

On the theoretical side, recent advances based on analytical work and multi-D models of stars of various mass, rotation rates and age, have started paving the way to a direct comparison with asteroseismic and spectropolarimetric data. Simulations coupling the angular evolution and magnetic activity on long time scale are in the making and should help explain the rotation history of stars. Acoustic and gravity modes excitation and propagation are now computed in detail and stellar cycles have now been found in many self-consistent convective dynamo simulations. General trends such as getting higher activity levels and shorter cycle period for faster rotating stars have been 
recovered. Still the exact dynamo mechanisms at work and resulting magnetic properties (topology, intensity, variability) for any given star are not yet fully elucidated. This comes about because the relative importance, spatial and temporal phasing and nonlinear interactions of the identified processes (e.g. shear in the tachocline, $\alpha$ and $\omega$ effects, stability of the magnetic wreaths, role of the meridional flow and turbulent pumping) at the origin of the organisation of the global (large scale) dynamo and the establishment of a cyclic magnetic activity vary. Thus the characterisation of the exact dependence of the stellar dynamo properties to stellar parameters such as mass, rotation/age and stratification is still an evolving understanding. In order to progress further theoretical models and high performance multi-D numerical simulations will benefit from having the following inputs/constraints:

- better constrained variations of the differential rotation in solar-like stars as a function of stellar parameters (mass, rotation/age, metallicity) and its latitudinal and radial profiles

- improved characterization of the $P_{c y c}$ vs $P_{\text {rot }}$ relation

- some assesment of the surface horizontal flows

- convective power and extent of the convective envelopes

- link between star spot numbers and stellar parameters, relative contribution to stellar brightness between dark spot, and bright faculae

- high cadence spectropolarimetric maps over the course of many years hence possibly mapping stellar cycles for many different stars

- calibration with asteroseismology of gyrochronology by studying stellar clusters of various ages

It is our hope that in the next decade or so, these multitude of information will become available and will aid the community in constraining and further developing theories and computational models of solar and stellar activity.

Acknowledgements This work was partly supported by ERC STARS2 207430 grant, ANR Toupies and IDEE grants, FP7 IRSES 269194 and SPACEINN 312844 programs, INSU/PNST. Funding for the Stellar Astrophysics Centre is provided by The Danish National Research Foundation (Grant agreement no.: DNRF106). The research is supported by the ASTERISK project (ASTERoseismic Investigations with SONG and Kepler) funded by the European Research Council (Grant agreement no.: 267864). CESSI is supported by the Ministry of Human Resource Development, Government of India and IISER Kolkata. A.S.B and R.A.G. acknowledge support by the CNES via GOLF, CoRoT annd Solar Orbiter grants. D.N. acknowledges support from the Ramanujan Fellowship (Department of Science and Technology) and a grant from the (United States) Asian Office of Aerospace Research and Development. A.S. Brun is grateful to both the University of Kyoto, RIMS and Prof. M. Yamada, K. Shibata, H. Isobe \& S. Takahiro for their invitation in Fall 2013 and to the University of California at Santa Barbara, KITP and the organisers of the waves and flow program in Spring 2014 where most of this paper was written.

\section{References}

Q.R. Ahmad, R.C. Allen, T.C. Andersen, J.D. Anglin, et al., Measurement of the Rate of $\nu_{e}+\mathrm{d}->\mathrm{p}+\mathrm{p}+e^{-}$Interactions Produced by ${ }^{8} \mathrm{~B}$ Solar Neutrinos at the Sudbury Neutrino Observatory. Physical Review Letters 87(7), 071301 (2001). doi:10.1103/PhysRevLett.87.071301

S. Aigrain, S. Hodgkin, J. Irwin, L. Hebb, M. Irwin, F. Favata, E. Moraux, F. Pont, The Monitor project: searching for occultations in young open clusters. MNRAS 375, 29-52 (2007). doi:10.1111/j.1365-2966.2006.11303.x 
S. Allain, Modelling the angular momentum evolution of low-mass stars with core-envelope decoupling. Astron. Astrophys. 333, 629-643 (1998)

N. Andronov, M.H. Pinsonneault, D.M. Terndrup, Mergers of Close Primordial Binaries. Astrophys. J. 646, 1160-1178 (2006). doi:10.1086/505127

M. Anguera Gubau, P.L. Palle, F. Perez Hernandez, C. Regulo, T. Roca Cortes, The low L solar p-mode spectrum at maximum and minimum solar activity. Astron. Astrophys. 255, 363-372 (1992)

T. Appourchaux, E. Michel, M. Auvergne, A. Baglin, et al., CoRoT sounds the stars: p-mode parameters of Sun-like oscillations on HD 49933. Astron. Astrophys. 488, 705-714 (2008). doi:10.1051/0004-6361:200810297

M. Asplund, N. Grevesse, A.J. Sauval, C. Allende Prieto, D. Kiselman, Line formation in solar granulation. IV. [O I], O I and $\mathrm{OH}$ lines and the photospheric $\mathrm{O}$ abundance. Astron. Astrophys. 417, 751-768 (2004). doi:10.1051/0004-6361:20034328

M. Asplund, N. Grevesse, A.J. Sauval, P. Scott, The Chemical Composition of the Sun. Annual Review of Astronomy and Astrophysics 47, 481-522 (2009). doi:10.1146/annurev.astro.46.060407.145222

J. Aubert, H. Amit, G. Hulot, Detecting thermal boundary control in surface flows from numerical dynamos. Physics of the Earth and Planetary Interiors 160, 143-156 (2007). doi:10.1016/j.pepi.2006.11.003

J. Aubert, J. Aurnou, J. Wicht, The magnetic structure of convection-driven numerical dynamos. Geophysical Journal International 172, 945-956 (2008). doi:10.1111/j.1365246X.2007.03693.x

K.C. Augustson, A.S. Brun, J. Toomre, Convection and Dynamo Action in B Stars, in $I A U$ Symposium, ed. by N.H. Brummell, A.S. Brun, M.S. Miesch, Y. Ponty IAU Symposium, vol. 271, 2011, pp. 361-362. doi:10.1017/S1743921311017790

K.C. Augustson, A.S. Brun, J. Toomre, Dynamo Action and Magnetic Cycles in F-type Stars. Astrophys. J. 777, 153 (2013). doi:10.1088/0004-637X/777/2/153

K.C. Augustson, B.P. Brown, A.S. Brun, M.S. Miesch, J. Toomre, Convection and Differential Rotation in F-type Stars. Astrophys. J. 756, 169 (2012). doi:10.1088/0004$637 \mathrm{X} / 756 / 2 / 169$

J. Aurnou, M. Heimpel, J. Wicht, The effects of vigorous mixing in a convective model of zonal flow on the ice giants. Icarus 190, 110-126 (2007). doi:10.1016/j.icarus.2007.02.024

H.W. Babcock, The Topology of the Sun's Magnetic Field and the 22-YEAR Cycle. Astrophys. J. 133, 572 (1961). doi:10.1086/147060

A. Baglin, M. Auvergne, L. Boisnard, T. Lam-Trong, P. Barge, C. Catala, M. Deleuil, E. Michel, W. Weiss, CoRoT: a High Precision Photometer for Stellar Ecolution and Exoplanet Finding, in 36th COSPAR Scientific Assembly. COSPAR, Plenary Meeting, vol. 36, 2006, p. 3749

S. Baliunas, R. Jastrow, Evidence for long-term brightness changes of solar-type stars. Nature 348, 520-523 (1990). doi:10.1038/348520a0

S.L. Baliunas, J.H. Horne, A. Porter, D.K. Duncan, J. Frazer, H. Lanning, A. Misch, J. Mueller, R.W. Noyes, D. Soyumer, A.H. Vaughan, L. Woodard, Time-series measurements of chromospheric CA II H and $\mathrm{K}$ emission in cool stars and the search for differential rotation. Astrophys. J. 294, 310-325 (1985). doi:10.1086/163299

S.L. Baliunas, R.A. Donahue, W.H. Soon, J.H. Horne, J. Frazer, et al. , Chromospheric variations in main-sequence stars. Astrophys. J. 438, 269-287 (1995). doi:10.1086/175072

S.L. Baliunas, E. Nesme-Ribes, D. Sokoloff, W.H. Soon, A Dynamo Interpretation of Stellar Activity Cycles. Astrophys. J. 460, 848 (1996). doi:10.1086/177014

J. Ballot, A.S. Brun, S. Turck-Chièze, Simulations of Turbulent Convection in Rotating Young Solarlike Stars: Differential Rotation and Meridional Circulation. Astrophys. J. 669, 11901208 (2007). doi:10.1086/521617

J. Ballot, S. Turck-Chièze, R.A. García, Seismic extraction of the convective extent in solarlike stars. The observational point of view. Astron. Astrophys. 423, 1051-1061 (2004). doi:10.1051/0004-6361:20035898

J. Ballot, L. Gizon, R. Samadi, G. Vauclair, et al., Accurate p-mode measurements of the G0V metal-rich CoRoT target HD 52265. Astron. Astrophys. 530, 97 (2011). doi:10.1051/0004$6361 / 201116547$

N.J. Balmforth, Solar pulsational stability. I - Pulsation-mode thermodynamics. MNRAS 255, 603-649 (1992)

N.J. Balmforth, D.O. Gough, W.J. Merryfield, Structural changes to the Sun through the solar 
cycle. MNRAS 278, 437-448 (1996)

C. Barban, S. Deheuvels, F. Baudin, T. Appourchaux, et al., Solar-like oscillations in HD 181420: data analysis of 156 days of CoRoT data. Astron. Astrophys. 506, 51-56 (2009). doi:10.1051/0004-6361/200911937

J.R. Barnes, A. Collier Cameron, J.-F. Donati, D.J. James, S.C. Marsden, P. Petit, The dependence of differential rotation on temperature and rotation. MNRAS 357, 1-5 (2005). doi:10.1111/j.1745-3933.2005.08587.x

S.A. Barnes, On the Rotational Evolution of Solar- and Late-Type Stars, Its Magnetic Origins, and the Possibility of Stellar Gyrochronology. Astrophys. J. 586, 464-479 (2003). doi:10.1086/367639

S.A. Barnes, Y.-C. Kim, Angular Momentum Loss from Cool Stars: An Empirical Expression and Connection to Stellar Activity. Astrophys. J. 721, 675-685 (2010). doi:10.1088/0004$637 \mathrm{X} / 721 / 1 / 675$

G. Basri, L.M. Walkowicz, N. Batalha, R.L. Gilliland, et al., Photometric Variability in Kepler Target Stars: The Sun Among Stars a First Look. Astrophys. J. Letters 713, 155-159 (2010). doi:10.1088/2041-8205/713/2/L155

G. Basri, L.M. Walkowicz, N. Batalha, R.L. Gilliland, et al., Photometric Variability in Kepler Target Stars. II. An Overview of Amplitude, Periodicity, and Rotation in First Quarter Data. Astron. J. 141, 20 (2011). doi:10.1088/0004-6256/141/1/20

S. Basu, H.M. Antia, Helium abundance in the solar envelope. MNRAS 276, 1402-1408 (1995)

S. Basu, N. Grevesse, S. Mathis, S. Turck-Chièze, Understanding the Internal Chemical Composition and Physical Processes of the Solar Interior. Space Sci. Rev. (2014). doi:10.1007/s11214-014-0035-9

P. Beaudoin, P. Charbonneau, E. Racine, P.K. Smolarkiewicz, Torsional Oscillations in a Global Solar Dynamo. Solar Phys. 282, 335-360 (2013). doi:10.1007/s11207-012-0150-2

T.R. Bedding, D. Huber, D. Stello, Y.P. Elsworth, et al., Solar-like Oscillations in Lowluminosity Red Giants: First Results from Kepler. Astrophys. J. Letters 713, 176-181 (2010). doi:10.1088/2041-8205/713/2/L176

J. Beer, S. Tobias, N. Weiss, An Active Sun Throughout the Maunder Minimum. Solar Phys. 181, 237-249 (1998)

K. Belkacem, R. Samadi, M.-J. Goupil, M.-A. Dupret, Stochastic excitation of nonradial modes. I. High-angular-degree p modes. Astron. Astrophys. 478, 163-174 (2008). doi:10.1051/0004-6361:20077775

K. Belkacem, R. Samadi, M. Goupil, L. Lefèvre, et al., Solar-Like Oscillations in a Massive Star. Science 324, 1540 (2009). doi:10.1126/science.1171913

K. Belkacem, M.J. Goupil, M.A. Dupret, R. Samadi, F. Baudin, A. Noels, B. Mosser, The underlying physical meaning of the nu max - nu c relation. Astron. Astrophys. 530, 142 (2011). doi:10.1051/0004-6361/201116490

C.P.M. Bell, T. Naylor, N.J. Mayne, R.D. Jeffries, S.P. Littlefair, Pre-main-sequence isochrones - II. Revising star and planet formation time-scales. MNRAS 434, 806-831 (2013). doi:10.1093/mnras/stt1075

E.E. Benevolenskaya, Double Magnetic Cycle of Solar Activity. Solar Phys. 161, 1-8 (1995). doi:10.1007/BF00732080

S.V. Berdyugina, Starspots: A Key to the Stellar Dynamo. Living Reviews in Solar Physics 2, 8 (2005). doi:10.12942/lrsp-2005-8

N. Bessolaz, A.S. Brun, Hunting for Giant Cells in Deep Stellar Convective Zones Using Wavelet Analysis. Astrophys. J. 728, 115 (2011). doi:10.1088/0004-637X/728/2/115

E. Böhm-Vitense, Über die Wasserstoffkonvektionszone in Sternen verschiedener Effektivtemperaturen und Leuchtkräfte. Mit 5 Textabbildungen. Zeitschrift fr Astrophysik 46, 108 (1958)

E. Böhm-Vitense, Chromospheric Activity in G and K Main-Sequence Stars, and What It Tells Us about Stellar Dynamos. Astrophys. J. 657, 486-493 (2007). doi:10.1086/510482

A. Bonaca, J.D. Tanner, S. Basu, W.J. Chaplin, et al., Calibrating Convective Properties of Solar-like Stars in the Kepler Field of View. Astrophys. J. Letters 755, 12 (2012). doi:10.1088/2041-8205/755/1/L12

W.J. Borucki, D. Koch, G. Basri, N. Batalha, T. Brown, et al., Kepler Planet-Detection Mission: Introduction and First Results. Science 327, 977 (2010). doi:10.1126/science.1185402

J. Bouvier, Observational Studies of Stellar Rotation, in EAS Publications Series. EAS Publications Series, vol. 62, 2013, pp. 143-168. doi:10.1051/eas/1362005

A.-M. Broomhall, D. Salabert, W.J. Chaplin, R.A. García, Y. Elsworth, R. Howe, S. Mathur, 
Misleading variations in estimated rotational frequency splittings of solar p modes: consequences for helioseismology and asteroseismology. MNRAS 422, 3564-3573 (2012). doi:10.1111/j.1365-2966.2012.20868.x

B.P. Brown, M.K. Browning, A.S. Brun, M.S. Miesch, J. Toomre, Rapidly Rotating Suns and Active Nests of Convection. Astrophys. J. 689, 1354-1372 (2008). doi:10.1086/592397

B.P. Brown, M.K. Browning, A.S. Brun, M.S. Miesch, J. Toomre, Persistent Magnetic Wreaths in a Rapidly Rotating Sun. Astrophys. J. 711, 424-438 (2010). doi:10.1088/0004637X/711/1/424

B.P. Brown, M.S. Miesch, M.K. Browning, A.S. Brun, J. Toomre, Magnetic Cycles in a Convective Dynamo Simulation of a Young Solar-type Star. Astrophys. J. 731, 69 (2011). doi:10.1088/0004-637X/731/1/69

T.M. Brown, The Metastable Dynamo Model of Stellar Rotational Evolution. Astrophys. J. 789, 101 (2014). doi:10.1088/0004-637X/789/2/101

T.M. Brown, C.A. Morrow, Depth and latitude dependence of solar rotation. Astrophys. J. Letters 314, 21-26 (1987). doi:10.1086/184843

T.M. Brown, R.L. Gilliland, R.W. Noyes, L.W. Ramsey, Detection of possible p-mode oscillations on Procyon. Astrophys. J. 368, 599-609 (1991). doi:10.1086/169725

M.K. Browning, Simulations of Dynamo Action in Fully Convective Stars. Astrophys. J. 676, 1262-1280 (2008). doi:10.1086/527432

M.K. Browning, A.S. Brun, J. Toomre, Simulations of Core Convection in Rotating AType Stars: Differential Rotation and Overshooting. Astrophys. J. 601, 512-529 (2004). doi:10.1086/380198

M.K. Browning, M.S. Miesch, A.S. Brun, J. Toomre, Dynamo Action in the Solar Convection Zone and Tachocline: Pumping and Organization of Toroidal Fields. Astrophys. J. Letters 648, 157-160 (2006). doi:10.1086/507869

A.S. Brun, On the interaction between differential rotation and magnetic fields in the Sun. Solar Phys. 220, 333-345 (2004). doi:10.1023/B:SOLA.0000031384.75850.68

A.S. Brun, J. Toomre, Turbulent Convection under the Influence of Rotation: Sustaining a Strong Differential Rotation. Astrophys. J. 570, 865-885 (2002). doi:10.1086/339228

A.S. Brun, M.K. Browning, J. Toomre, Simulations of Core Convection in Rotating A-Type Stars: Magnetic Dynamo Action. Astrophys. J. 629, 461-481 (2005). doi:10.1086/430430

A.S. Brun, M.S. Miesch, J. Toomre, Global-Scale Turbulent Convection and Magnetic Dynamo Action in the Solar Envelope. Astrophys. J. 614, 1073-1098 (2004). doi:10.1086/423835

A.S. Brun, M.S. Miesch, J. Toomre, Modeling the Dynamical Coupling of Solar Convection with the Radiative Interior. Astrophys. J. 742, 79 (2011). doi:10.1088/0004$637 \mathrm{X} / 742 / 2 / 79$

A.S. Brun, S. Turck-Chièze, P. Morel, Standard Solar Models in the Light of New Helioseismic Constraints. I. The Solar Core. Astrophys. J. 506, 913-925 (1998). doi:10.1086/306271

A.S. Brun, S. Turck-Chièze, J.P. Zahn, Standard Solar Models in the Light of New Helioseismic Constraints. II. Mixing below the Convective Zone. Astrophys. J. 525, 1032-1041 (1999). doi:10.1086/307932

A.S. Brun, M.K. Browning, M. Dikpati, H. Hotta, A. Strugarek, Recent Advances on Solar Global Magnetism and Variability. Space Sci. Rev. (2013). doi:10.1007/s11214-013-0028-0

A.S. Brun, S. Mathis, Angular momentum transport in stars: from short to long time scales. Extraterrestrial seismology book, (2014)

H. Bruntt, Accurate fundamental parameters of CoRoT asteroseismic targets. The solar-like stars HD 49933, HD 175726, HD 181420, and HD 181906. Astron. Astrophys. 506, 235-244 (2009). doi:10.1051/0004-6361/200911925

P.J. Bushby, Zonal flows and grand minima in a solar dynamo model. MNRAS 371, 772-780 (2006). doi:10.1111/j.1365-2966.2006.10706.x

T. Cai, K.L. Chan, L. Deng, Numerical simulation of core convection by a multi-layer semiimplicit spherical spectral method. Journal of Computational Physics 230, 8698-8712 (2011). doi:10.1016/j.jcp.2011.08.014

T.L. Campante, R. Handberg, S. Mathur, T. Appourchaux, et al., Asteroseismology from multi-month Kepler photometry: the evolved Sun-like stars KIC 10273246 and KIC 10920273. Astron. Astrophys. 534, 6 (2011). doi:10.1051/0004-6361/201116620

F. Carrier, F. Bouchy, P. Eggenberger, New Seismological Results on the G0 Iv Bootis, in Asteroseismology Across the HR Diagram, ed. by M.J. Thompson, M.S. Cunha, M.J.P.F.G. Monteiro, 2003, pp. 315-318

F. Cattaneo, On the Origin of Magnetic Fields in the Quiet Photosphere. Astrophys. J. Letters 
515, 39-42 (1999). doi:10.1086/311962

K.H. Chan, K. Zhang, L. Li, X. Liao, A new generation of convection-driven spherical dynamos using EBE finite element method. Physics of the Earth and Planetary Interiors 163, 251265 (2007). doi:10.1016/j.pepi.2007.04.017

S. Chandrasekhar, Letter to the Editor: a General Variational Principle Governing the Radial and the Non-Radial Oscillations of Gaseous Masses. Astrophys. J. 138, 896 (1963). doi:10.1086/147694

W.J. Chaplin, Y. Elsworth, R. Howe, G.R. Isaak, C.P. McLeod, B.A. Miller, H.B. van der Raay, S.J. Wheeler, R. New, BiSON Performance. Solar Phys. 168, 1-18 (1996)

W.J. Chaplin, Y. Elsworth, G.R. Isaak, R. Lines, C.P. McLeod, B.A. Miller, R. New, An analysis of solar p-mode frequencies extracted from BiSON data: 1991-1996. MNRAS 300, 1077-1090 (1998). doi:10.1046/j.1365-8711.1998.01999.x

W.J. Chaplin, Y. Elsworth, G.R. Isaak, B.A. Miller, R. New, Variations in the excitation and damping of low-l solar p modes over the solar activity cycle*. MNRAS 313, 32-42 (2000). doi:10.1046/j.1365-8711.2000.03176.x

W.J. Chaplin, G. Houdek, Y. Elsworth, D.O. Gough, G.R. Isaak, R. New, On model predictions of the power spectral density of radial solar p modes. MNRAS 360, 859-868 (2005). doi:10.1111/j.1365-2966.2005.09041.x

W.J. Chaplin, Y. Elsworth, G. Houdek, R. New, On prospects for sounding activity cycles of Sun-like stars with acoustic modes. MNRAS 377, 17-29 (2007). doi:10.1111/j.13652966.2007.11581.x

W.J. Chaplin, T. Appourchaux, Y. Elsworth, R.A. García, G. Houdek, et al., The Asteroseismic Potential of Kepler: First Results for Solar-Type Stars. Astrophys. J. Letters 713, 169-175 (2010). doi:10.1088/2041-8205/713/2/L169

W.J. Chaplin, T.R. Bedding, A. Bonanno, A. Broomhall, R.A. García, et al., Evidence for the Impact of Stellar Activity on the Detectability of Solar-like Oscillations Observed by Kepler. Astrophys. J. Letters 732, 5 (2011a). doi:10.1088/2041-8205/732/1/L5

W.J. Chaplin, H. Kjeldsen, T.R. Bedding, J. Christensen-Dalsgaard, et al., Predicting the Detectability of Oscillations in Solar-type Stars Observed by Kepler. Astrophys. J. 732, 54 (2011b). doi:10.1088/0004-637X/732/1/54

P. Charbonneau, Dynamo Models of the Solar Cycle. Living Reviews in Solar Physics 7, 3 (2010). doi:10.12942/lrsp-2010-3

P. Charbonneau, Solar and Stellar Dynamos. Society for Astronomical Sciences Annual Symposium 39(2013a). doi:10.1007/978-3-642-32093-4

P. Charbonneau, Stellar Dynamos. Society for Astronomical Sciences Annual Symposium 39, $187(2013 \mathrm{~b})$

P. Charbonneau, M. Dikpati, Stochastic Fluctuations in a Babcock-Leighton Model of the Solar Cycle. Astrophys. J. 543, 1027-1043 (2000). doi:10.1086/317142

P. Charbonneau, K.B. MacGregor, Angular Momentum Transport in Magnetized Stellar Radiative Zones. II. The Solar Spin-down. Astrophys. J. 417, 762 (1993). doi:10.1086/173357

U.R. Christensen, Dynamo Scaling Laws and Applications to the Planets. Space Sci. Rev. 152, 565-590 (2010). doi:10.1007/s11214-009-9553-2

U.R. Christensen, J. Aubert, Scaling properties of convection-driven dynamos in rotating spherical shells and application to planetary magnetic fields. Geophysical Journal International 166, 97-114 (2006). doi:10.1111/j.1365-246X.2006.03009.x

U.R. Christensen, V. Holzwarth, A. Reiners, Energy flux determines magnetic field strength of planets and stars. Nature 457, 167-169 (2009). doi:10.1038/nature07626

J. Christensen-Dalsgaard, Physics of solar-like oscillations. Solar Phys. 220, 137-168 (2004). doi:10.1023/B:SOLA.0000031392.43227.7d

J. Christensen-Dalsgaard, D.O. Gough, Is the sun helium-deficient. Nature 288, 544-547 (1980). doi:10.1038/288544a0

J. Christensen-Dalsgaard, G. Houdek, Prospects for asteroseismology. Astrophysics and Space Science 328, 51-66 (2010). doi:10.1007/s10509-009-0227-z

J. Christensen-Dalsgaard, D.O. Gough, M.J. Thompson, The depth of the solar convection zone. Astrophys. J. 378, 413-437 (1991). doi:10.1086/170441

J. Christensen-Dalsgaard, W. Dappen, S.V. Ajukov, E.R. Anderson, H.M. Antia, et al. , The Current State of Solar Modeling. Science 272, 1286-1292 (1996). doi:10.1126/science.272.5266.1286

J. Christensen-Dalsgaard, M.J.P.F.G. Monteiro, M. Rempel, M.J. Thompson, A more real- 
istic representation of overshoot at the base of the solar convective envelope as seen by helioseismology. MNRAS 414, 1158-1174 (2011). doi:10.1111/j.1365-2966.2011.18460.x

A. Claverie, G.R. Isaak, C.P. McLeod, H.B. van der Raay, T.R. Cortes, Solar structure from global studies of the 5-minute oscillation. Nature 282, 591-594 (1979). doi:10.1038/282591a0

A. Collier Cameron, Differential rotation on rapidly rotating stars. Astronomische Nachrichten 328, 1030 (2007). doi:10.1002/asna.200710880

S.R. Cranmer, S.H. Saar, Testing a Predictive Theoretical Model for the Mass Loss Rates of Cool Stars. Astrophys. J. 741, 54 (2011). doi:10.1088/0004-637X/741/1/54

O.L. Creevey, F. Thévenin, S. Basu, W.J. Chaplin, L. Bigot, Y. Elsworth, D. Huber, M.J.P.F.G. Monteiro, A. Serenelli, A large sample of calibration stars for Gaia: log g from Kepler and CoRoT. ArXiv e-prints (2013)

M. Cuntz, W. Rammacher, P. Ulmschneider, Z.E. Musielak, S.H. Saar, Two-Component Theoretical Chromosphere Models for K Dwarfs of Different Magnetic Activity: Exploring the Ca II Emission-Stellar Rotation Relationship. Astrophys. J. 522, 1053-1068 (1999). doi:10.1086/307689

M. Dasi-Espuig, S.K. Solanki, N.A. Krivova, R. Cameron, T. Peñuela, Sunspot group tilt angles and the strength of the solar cycle. Astron. Astrophys. 518, 7 (2010). doi:10.1051/0004$6361 / 201014301$

J. de Ridder, C. Barban, F. Baudin, F. Carrier, et al., Non-radial oscillation modes with long lifetimes in giant stars. Nature 459, 398-400 (2009). doi:10.1038/nature08022

S. Deheuvels, R.A. García, W.J. Chaplin, S.e. Basu, Seismic Evidence for a Rapidly Rotating Core in a Lower-giant-branch Star Observed with Kepler. Astrophys. J. 756, 19 (2012). doi:10.1088/0004-637X/756/1/19

P. Delorme, A. Collier Cameron, L. Hebb, J. Rostron, T.A. Lister, A.J. Norton, D. Pollacco, R.G. West, Stellar rotation in the Hyades and Praesepe: gyrochronology and braking timescale. MNRAS 413, 2218-2234 (2011). doi:10.1111/j.1365-2966.2011.18299.x

P.A. Denissenkov, M. Pinsonneault, D.M. Terndrup, G. Newsham, Angular Momentum Transport in Solar-type Stars: Testing the Timescale for Core-Envelope Coupling. Astrophys. J. 716, 1269-1287 (2010). doi:10.1088/0004-637X/716/2/1269

M.L. DeRosa, A.S. Brun, J.T. Hoeksema, Solar Magnetic Field Reversals and the Role of Dynamo Families. Astrophys. J. 757, 96 (2012). doi:10.1088/0004-637X/757/1/96

M. Dikpati, P. Charbonneau, A Babcock-Leighton Flux Transport Dynamo with Solar-like Differential Rotation. Astrophys. J. 518, 508-520 (1999). doi:10.1086/307269

O. Do Cao, A.S. Brun, Effects of turbulent pumping on stellar activity cycles. Astronomische Nachrichten 332, 907 (2011). doi:10.1002/asna.201111623

J.D. Do Nascimento, J.S. da Costa, J.R. de Medeiros, Rotation and lithium abundance of solaranalog stars. Theoretical analysis of observations. Astron. Astrophys. 519, 101 (2010). doi:10.1051/0004-6361/200811026

J.-D. do Nascimento Jr., R.A. García, S. Mathur, F. Anthony, S.A. Barnes, S. Meibom, J.S. da Costa, M. Castro, D. Salabert, T. Ceillier, Rotation Periods and Ages of Solar Analogs and Solar Twins Revealed by the Kepler Mission. Astrophys. J. Letters 790, 23 (2014). doi:10.1088/2041-8205/790/2/L23

W. Dobler, M. Stix, A. Brandenburg, Magnetic Field Generation in Fully Convective Rotating Spheres. Astrophys. J. 638, 336-347 (2006). doi:10.1086/498634

V. Domingo, B. Fleck, A.I. Poland, The SOHO Mission: an Overview. Solar Phys. 162, 1-37 (1995)

R.A. Donahue, S.H. Saar, S.L. Baliunas, A Relationship between Mean Rotation Period in Lower Main-Sequence Stars and Its Observed Range. Astrophys. J. 466, 384 (1996). doi:10.1086/177517

J.-F. Donati, J.D. Landstreet, Magnetic Fields of Nondegenerate Stars. Annual Review of Astronomy and Astrophysics 47, 333-370 (2009). doi:10.1146/annurev-astro-082708-101833

J.-F. Donati, M. Semel, B.D. Carter, D.E. Rees, A. Collier Cameron, Spectropolarimetric observations of active stars. MNRAS 291, 658 (1997)

J.-F. Donati, I.D. Howarth, M.M. Jardine, P. Petit, C. Catala, J.D. Landstreet, J.-C. Bouret, E. Alecian, J.R. Barnes, T. Forveille, F. Paletou, N. Manset, The surprising magnetic topology of $\tau$ Sco: fossil remnant or dynamo output? MNRAS 370, 629-644 (2006). doi:10.1111/j.1365-2966.2006.10558.x

S. D'Silva, A.R. Choudhuri, A theoretical model for tilts of bipolar magnetic regions. Astron. Astrophys. 272, 621 (1993) 
C. Dubé, P. Charbonneau, Stellar Dynamos and Cycles from Numerical Simulations of Convection. Astrophys. J. 775, 69 (2013). doi:10.1088/0004-637X/775/1/69

B.R. Durney, J. Latour, On the angular momentum loss of late-type stars. Geophysical and Astrophysical Fluid Dynamics 9, 241-255 (1978)

W.A. Dziembowski, P.R. Goode, Helioseismic Probing of Solar Variability: The Formalism and Simple Assessments. Astrophys. J. 600, 464-479 (2004). doi:10.1086/379708

W.A. Dziembowski, P.R. Goode, Sources of Oscillation Frequency Increase with Rising Solar Activity. Astrophys. J. 625, 548-555 (2005). doi:10.1086/429712

A. Eff-Darwich, S.G. Korzennik, The Dynamics of the Solar Radiative Zone. Solar Phys., 149 (2012). doi:10.1007/s11207-012-0048-z

S. Ekström, C. Georgy, P. Eggenberger, G. Meynet, N. Mowlavi, A. Wyttenbach, A. Granada, T. Decressin, R. Hirschi, U. Frischknecht, C. Charbonnel, A. Maeder, Grids of stellar models with rotation. I. Models from 0.8 to $120 \mathrm{M}$ at solar metallicity $(\mathrm{Z}=0.014)$. Astron. Astrophys. 537, 146 (2012). doi:10.1051/0004-6361/201117751

A.S. Endal, S. Sofia, Rotation in solar-type stars. I - Evolutionary models for the spin-down of the sun. Astrophys. J. 243, 625-640 (1981). doi:10.1086/158628

C.R. Epstein, M.H. Pinsonneault, How Good a Clock is Rotation? The Stellar Rotation-MassAge Relationship for Old Field Stars. Astrophys. J. 780, 159 (2014). doi:10.1088/0004$637 \mathrm{X} / 780 / 2 / 159$

Y. Fan, Magnetic Fields in the Solar Convection Zone. Living Reviews in Solar Physics 6, 4 (2009). doi:10.12942/lrsp-2009-4

S. Fauve, F. Pétrélis, Scaling laws of turbulent dynamos. Comptes Rendus Physique 8, 87-92 (2007). doi:10.1016/j.crhy.2006.12.011

N.A. Featherstone, M.K. Browning, A.S. Brun, J. Toomre, Effects of Fossil Magnetic Fields on Convective Core Dynamos in A-type Stars. Astrophys. J. 705, 1000-1018 (2009). doi:10.1088/0004-637X/705/1/1000

E.D. Feigelson, T. Montmerle, High-Energy Processes in Young Stellar Objects. Annual Review of Astronomy and Astrophysics 37, 363-408 (1999). doi:10.1146/annurev.astro.37.1.363

S.T. Fletcher, A. Broomhall, D. Salabert, S. Basu, W.J. Chaplin, Y. Elsworth, R.A. Garcia, R. New, A Seismic Signature of a Second Dynamo? Astrophys. J. Letters 718, 19-22 (2010). doi:10.1088/2041-8205/718/1/L19

E. Fossat, B. Gelly, G. Grec, M. Pomerantz, Search for Solar P-Mode Frequency Changes Between 1980 and 1985. Astron. Astrophys. 177, 47 (1987)

C. Fröhlich, Total Solar Irradiance Observations. Surveys in Geophysics 33, 453-473 (2012). doi:10.1007/s10712-011-9168-5

A.H. Gabriel, G. Grec, J. Charra, J.-M. Robillot, et al., Global Oscillations at Low Frequency from the SOHO Mission (GOLF). Solar Phys. 162, 61-99 (1995)

F. Gallet, J. Bouvier, Improved angular momentum evolution model for solar-like stars. Astron. Astrophys. 556, 36 (2013). doi:10.1051/0004-6361/201321302

P. Garaud, P. Bodenheimer, Gyroscopic Pumping of Large-scale Flows in Stellar Interiors and Application to Lithium-dip Stars. Astrophys. J. 719, 313-334 (2010). doi:10.1088/0004$637 \mathrm{X} / 719 / 1 / 313$

R.A. García, Solar-Like Stars Observed by Kepler: An Incredible Adventure, in Stellar Pulsations: Impact of New Instrumentation and New Insights, ed. by J.C. Suárez, R. Garrido, L.A. Balona, J. Christensen-Dalsgaard Advances in Solid State Physics, vol. 31, 2013, p. 171

R.A. García, T. Corbard, W.J. Chaplin, S. Couvidat, A. Eff-Darwich, S.J. Jiménez-Reyes, S.G. Korzennik, J. Ballot, P. Boumier, E. Fossat, C.J. Henney, R. Howe, M. Lazrek, J. Lochard, P.L. Pallé, S. Turck-Chièze, About the rotation of the solar radiative interior. Solar Phys. 220, 269-285 (2004). doi:10.1023/B:SOLA.0000031395.90891.ce

R.A. García, S. Mathur, J. Ballot, A. Eff-Darwich, S.J. Jiménez-Reyes, S.G. Korzennik, Influence of Low-Degree High-Order p-Mode Splittings on the Solar Rotation Profile. Solar Phys. 251, 119-133 (2008). doi:10.1007/s11207-008-9144-5

R.A. García, C. Régulo, R. Samadi, J. Ballot, et al., Solar-like oscillations with low amplitude in the CoRoT target HD 181906. Astron. Astrophys. 506, 41-50 (2009). doi:10.1051/0004$6361 / 200911910$

R.A. García, S. Mathur, D. Salabert, J. Ballot, C. Régulo, T.S. Metcalfe, A. Baglin, CoRoT Reveals a Magnetic Activity Cycle in a Sun-Like Star. Science 329, 1032 (2010). doi:10.1126/science.1191064

R.A. García, S. Hekker, D. Stello, J. Gutiérrez-Soto, et al., Preparation of Kepler 
light curves for asteroseismic analyses. MNRAS 414, 6-10 (2011). doi:10.1111/j.17453933.2011.01042.x

R.A. García, T. Ceillier, T.L. Campante, G.R. Davies, S. Mathur, PE11 Team of Kepler WG\#1, Fast Rotating Solar-like Stars Using Asteroseismic Datasets, in Progress in Solar/Stellar Physics with Helio- and Asteroseismology, ed. by H. Shibahashi, M. Takata, A.E. Lynas-Gray Astronomical Society of the Pacific Conference Series, vol. 462, 2012, p. 133

R.A. García, D. Salabert, S. Mathur, C. Régulo, J. Ballot, G.R. Davies, A. Jiménez, R. Simoniello, Towards solar activity maximum 24 as seen by GOLF and VIRGO/SPM instruments. Journal of Physics Conference Series 440(1), 012020 (2013). doi:10.1088/1742$6596 / 440 / 1 / 012020$

R.A. Garcia, T. Ceillier, D. Salabert, S. Mathur, J.L. van Saders, M. Pinsonneault, J. Ballot, P.G. Beck, S. Bloemen, T.L. Campante, G.R. Davies, J.-D. do Nascimento Jr., S. Mathis, T.S. Metcalfe, M.B. Nielsen, J.C. Suarez, W.J. Chaplin, A. Jimenez, C. Karoff, Rotation and magnetism of Kepler pulsating solar-like stars. Towards asteroseismically calibrated age-rotation relations. ArXiv e-prints (2014)

T. Gastine, L. Duarte, J. Wicht, Dipolar versus multipolar dynamos: the influence of the background density stratification. Astron. Astrophys. 546, 19 (2012). doi:10.1051/0004$6361 / 201219799$

T. Gastine, J. Wicht, J.M. Aurnou, Zonal flow regimes in rotating anelastic spherical shells: An application to giant planets. Icarus 225, 156-172 (2013). doi:10.1016/j.icarus.2013.02.031

T. Gastine, R.K. Yadav, J. Morin, A. Reiners, J. Wicht, From solar-like to antisolar differential rotation in cool stars. MNRAS 438, 76-80 (2014). doi:10.1093/mnrasl/slt162

B. Gelly, M. Lazrek, G. Grec, A. Ayad, F.X. Schmider, C. Renaud, D. Salabert, E. Fossat, Solar p-modes from 1979 days of the GOLF experiment. Astron. Astrophys. 394, 285-297 (2002). doi:10.1051/0004-6361:20021106

C. Georgy, S. Ekström, P. Eggenberger, G. Meynet, L. Haemmerlé, A. Maeder, A. Granada, J.H. Groh, R. Hirschi, N. Mowlavi, N. Yusof, C. Charbonnel, T. Decressin, F. Barblan, Grids of stellar models with rotation. III. Models from 0.8 to $120 \mathrm{M}$ at a metallicity $\mathrm{Z}=$ 0.002. Astron. Astrophys. 558, 103 (2013). doi:10.1051/0004-6361/201322178

M. Ghizaru, P. Charbonneau, P.K. Smolarkiewicz, Magnetic Cycles in Global Large-eddy Simulations of Solar Convection. Astrophys. J. Letters 715, 133-137 (2010). doi:10.1088/20418205/715/2/L133

M.S. Giampapa, Stellar Analogs of Solar Activity: the Sun in a Stellar Context, in Saas-Fee Advanced Course 34: The Sun, Solar Analogs and the Climate, ed. by J.D. Haigh, M. Lockwood, M.S. Giampapa, I. Rüedi, M. Güdel, W. Schmutz, 2005, pp. 307-415

M.S. Giampapa, J.C. Hall, R.R. Radick, S.L. Baliunas, A Survey of Chromospheric Activity in the Solar-Type Stars in the Open Cluster M67. Astrophys. J. 651, 444-461 (2006). doi:10.1086/507624

P.A. Gilman, Dynamically consistent nonlinear dynamos driven by convection in a rotating spherical shell. II - Dynamos with cycles and strong feedbacks. Astrophys. J. Supp. 53, 243-268 (1983). doi:10.1086/190891

P.A. Gilman, J. Miller, Dynamically consistent nonlinear dynamos driven by convection in a rotating spherical shell. Astrophys. J. Supp. 46, 211-238 (1981). doi:10.1086/190743

P.A. Gilman, C.A. Morrow, E.E. Deluca, Angular momentum transport and dynamo action in the sun - Implications of recent oscillation measurements. Astrophys. J. 338, 528-537 (1989). doi:10.1086/167215

G. Glatzmaier, M. Evonuk, T. Rogers, Differential rotation in giant planets maintained by density-stratified turbulent convection. Geophysical and Astrophysical Fluid Dynamics 103, 31-51 (2009). doi:10.1080/03091920802221245

G.A. Glatzmaier, Numerical simulations of stellar convective dynamos. I - The model and method. Journal of Computational Physics 55, 461-484 (1984). doi:10.1016/00219991(84)90033-0

G.A. Glatzmaier, Numerical simulations of stellar convective dynamos. II - Field propagation in the convection zone. Astrophys. J. 291, 300-307 (1985a). doi:10.1086/163069

G.A. Glatzmaier, Numerical simulations of stellar convective dynamos. III - At the base of the convection zone. Geophysical and Astrophysical Fluid Dynamics 31, 137-150 (1985b). doi:10.1080/03091928508219267

G.A. Glatzmaier, P.A. Gilman, Compressible convection in a rotating spherical shell. V Induced differential rotation and meridional circulation. Astrophys. J. 256, 316-330 (1982). 
doi:10.1086/159909

G.A. Glatzmaier, P.H. Roberts, Rotation and Magnetism of Earth's Inner Core. Science 274, 1887-1891 (1996). doi:10.1126/science.274.5294.1887

P. Goldreich, D.A. Keeley, Solar seismology. II - The stochastic excitation of the solar p-modes by turbulent convection. Astrophys. J. 212, 243-251 (1977). doi:10.1086/155043

P. Goldreich, N. Murray, G. Willette, P. Kumar, Implications of solar p-mode frequency shifts. Astrophys. J. 370, 752-762 (1991). doi:10.1086/169858

P. Gondoin, Dynamo regime transition among Sun-like stars in M 34. A time evolution model of X-ray activity on the main sequence. Astron. Astrophys. 546, 117 (2012). doi:10.1051/0004-6361/201219823

L. Goudard, E. Dormy, Relations between the dynamo region geometry and the magnetic behavior of stars and planets. EPL (Europhysics Letters) 83, 59001 (2008). doi:10.1209/0295$5075 / 83 / 59001$

D. Gough, Mixing-length theory for pulsating stars. Astrophys. J. 214, 196-213 (1977a). doi:10.1086/155244

D. Gough, The Current State of Stellar Mixing-length Theory, in Problems of Stellar Convection, ed. by E.A. Spiegel, J.-P. Zahn Lecture Notes in Physics, Berlin Springer Verlag, vol. 71,1977 b, pp. $15-56$

D. Gough, New data from solar oscillations. Nature 274, 739 (1978). doi:10.1038/274739a0

D. Gough, How Oblate Is the Sun? Science 337, 1611 (2012). doi:10.1126/science.1226988

D. Gough, What Have We Learned from Helioseismology, What Have We Really Learned, and What Do We Aspire to Learn? Solar Phys. 287, 9-41 (2013). doi:10.1007/s11207-0120099-1

D.O. Gough, The Anelastic Approximation for Thermal Convection. Journal of Atmospheric Sciences 26, 448-456 (1969). doi:10.1175/1520-0469(1969)026;0448:TAAFTC ¿2.0.CO;2

D.O. Gough, Immediate and long-term prospects for helioseismology. Advances in Space Research 4, 85-102 (1984a). doi:10.1016/0273-1177(84)90370-3

D.O. Gough, Solar Inverse Theory, in Solar seismology from space, vol. 84, ed. by R.K. Ulrich, J. Harvey, E.. RHodes, J. Toomre, 1984b, pp. 49-78

D.O. Gough, Comments on Helioseismic Inference, in Progress of Seismology of the Sun and Stars, ed. by Y. Osaki, H. Shibahashi Lecture Notes in Physics, Berlin Springer Verlag, vol. 367,1990 , p. 283. doi:10.1007/3-540-53091-6

D.O. Gough, M.J. Thompson, Magnetic Perturbations to Stellar Oscillation Eigenfrequencies, in Advances in Helio- and Asteroseismology, ed. by J. Christensen-Dalsgaard, S. Frandsen IAU Symposium, vol. 123, 1988, p. 155

S.G. Gregory, J.-F. Donati, J. Morin, G.A.J. Hussain, N.J. Mayne, L.A. Hillenbrand, M. Jardine, Can We Predict the Global Magnetic Topology of a Pre-main-sequence Star from Its Position in the Hertzsprung-Russell Diagram? Astrophys. J. 755, 97 (2012). doi:10.1088/0004-637X/755/2/97

E. Grote, F.H. Busse, Dynamics of convection and dynamos in rotating spherical fluid shells. Fluid Dynamics Research 28, 349-368 (2001). doi:10.1016/S0169-5983(01)00004-1

F. Grundahl, J. Christensen-Dalsgaard, P.L. Pallé, M.F. Andersen, S. Frandsen, K. Harpsøe, U.G. Jørgensen, H. Kjeldsen, P.K. Rasmussen, J. Skottfelt, A.N. Sørensen, A.T. Hage, Stellar Observations Network Group: The Prototype Is Nearly Ready, in IAU Symposium, ed. by J.A. Guzik, W.J. Chaplin, G. Handler, A. Pigulski IAU Symposium, vol. 301, 2014, pp. 69-75. doi:10.1017/S1743921313014117

M. Güdel, X-ray astronomy of stellar coronae. Astronomy and Astrophysics Review 12, 71-237 (2004). doi:10.1007/s00159-004-0023-2

M. Güdel, The Sun in Time: Activity and Environment. Living Reviews in Solar Physics 4, 3 (2007). doi:10.12942/lrsp-2007-3

G. Guerrero, E.M. de Gouveia Dal Pino, Turbulent magnetic pumping in a BabcockLeighton solar dynamo model. Astron. Astrophys. 485, 267-273 (2008). doi:10.1051/00046361:200809351

G. Guerrero, P.K. Smolarkiewicz, A.G. Kosovichev, N.N. Mansour, Differential Rotation in Solar-like Stars from Global Simulations. Astrophys. J. 779, 176 (2013). doi:10.1088/0004$637 \mathrm{X} / 779 / 2 / 176$

D.A. Haber, B.W. Hindman, J. Toomre, R.S. Bogart, R.M. Larsen, F. Hill, Evolving Submerged Meridional Circulation Cells within the Upper Convection Zone Revealed by RingDiagram Analysis. Astrophys. J. 570, 855-864 (2002). doi:10.1086/339631

G.E. Hale, F. Ellerman, S.B. Nicholson, A.H. Joy, The Magnetic Polarity of Sun-Spots. As- 
trophys. J. 49, 153 (1919). doi:10.1086/142452

J.C. Hall, Stellar Chromospheric Activity. Living Reviews in Solar Physics 5, 2 (2008). doi:10.12942/lrsp-2008-2

J.C. Hall, G.W. Lockwood, B.A. Skiff, The Activity and Variability of the Sun and Sunlike Stars. I. Synoptic Ca II H and K Observations. Astron. J. 133, 862-881 (2007). doi:10.1086/510356

J.C. Hall, G.W. Henry, G.W. Lockwood, B.A. Skiff, S.H. Saar, The Activity and Variability of the Sun and Sun-Like Stars. II. Contemporaneous Photometry and Spectroscopy of Bright Solar Analogs. Astron. J. 138, 312-322 (2009). doi:10.1088/0004-6256/138/1/312

J.D. Hartman, B.S. Gaudi, M.H. Pinsonneault, K.Z. Stanek, M.J. Holman, B.A. McLeod, S. Meibom, J.A. Barranco, J.S. Kalirai, Deep MMT Transit Survey of the Open Cluster M37. III. Stellar Rotation at 550 Myr. Astrophys. J. 691, 342-364 (2009). doi:10.1088/0004$637 \mathrm{X} / 691 / 1 / 342$

J.D. Hartman, G.Á. Bakos, G. Kovács, R.W. Noyes, A large sample of photometric rotation periods for FGK Pleiades stars. MNRAS 408, 475-489 (2010). doi:10.1111/j.13652966.2010.17147.x

J.D. Hartman, G.Á. Bakos, R.W. Noyes, B. Sipőcz, G. Kovács, T. Mazeh, A. Shporer, A. Pál, A Photometric Variability Survey of Field K and M Dwarf Stars with HATNet. Astron. J. 141, 166 (2011). doi:10.1088/0004-6256/141/5/166

J.W. Harvey, -, in Probing the depths of a star: the study of solar oscillations, vol. 400, ed. by R.W. Noyes, E.J. Rhodes, JPL, 1985, p. 327. JPL

D.H. Hathaway, The Solar Cycle. Living Reviews in Solar Physics 7, 1 (2010). doi:10.12942/lrsp-2010-1

G. Hazra, B.B. Karak, A.R. Choudhuri, Is a Deep One-cell Meridional Circulation Essential for the Flux Transport Solar Dynamo? Astrophys. J. 782, 93 (2014). doi:10.1088/0004$637 \mathrm{X} / 782 / 2 / 93$

M.H. Heimpel, J.M. Aurnou, F.M. Al-Shamali, N. Gomez Perez, A numerical study of dynamo action as a function of spherical shell geometry. Earth and Planetary Science Letters 236, 542-557 (2005). doi:10.1016/j.epsl.2005.04.032

S. Hekker, T. Kallinger, F. Baudin, J. De Ridder, C. Barban, F. Carrier, A.P. Hatzes, W.W. Weiss, A. Baglin, Characteristics of solar-like oscillations in red giants observed in the CoRoT exoplanet field. Astron. Astrophys. 506, 465-469 (2009). doi:10.1051/0004$6361 / 200911858$

S. Hekker, A. Broomhall, W.J. Chaplin, Y.P. Elsworth, S.T. Fletcher, R. New, T. Arentoft, P. Quirion, H. Kjeldsen, The Octave (Birmingham-Sheffield Hallam) automated pipeline for extracting oscillation parameters of solar-like main-sequence stars. MNRAS 402, 20492059 (2010). doi:10.1111/j.1365-2966.2009.16030.x

S. Hekker, Y. Elsworth, J. De Ridder, B. Mosser, R.A. García, et al., Solar-like oscillations in red giants observed with Kepler: comparison of global oscillation parameters from different methods. Astron. Astrophys. 525, 131 (2011). doi:10.1051/0004-6361/201015185

S. Hekker, Y. Elsworth, B. Mosser, T. Kallinger, S. Basu, W.J. Chaplin, D. Stello, Asteroseismic surface gravity for evolved stars. Astron. Astrophys. 556, 59 (2013). doi:10.1051/0004$6361 / 201321630$

A. Hempelmann, J.H.M.M. Schmitt, K. Stẹpień, Coronal X-ray emission of cool stars in relation to chromospheric activity and magnetic cycles. Astron. Astrophys. 305, 284 (1996)

W. Herschel, Observations Tending to Investigate the Nature of the Sun, in Order to Find the Causes or Symptoms of Its Variable Emission of Light and Heat; With Remarks on the Use That May Possibly be Drawn from Solar Observations. Royal Society of London Philosophical Transactions Series I 91, 265-318 (1801)

R. Hollerbach, C.A. Jones, Influence of the Earth's inner core on geomagnetic fluctuations and reversals. Nature 365, 541-543 (1993). doi:10.1038/365541a0

H. Hotta, T. Yokoyama, Modeling of Differential Rotation in Rapidly Rotating Solar-type Stars. Astrophys. J. 740, 12 (2011). doi:10.1088/0004-637X/740/1/12

G. Houdek, Pulsation of Solar-type Stars, PhD thesis, "Ph.D. Thesis, Formal- und Naturwisseschaftliche Fakult" at der Universität Wien, (1996)", 1996

G. Houdek, Asteroseismic Helium Abundance Determination, in Equation-of-State and PhaseTransition in Models of Ordinary Astrophysical Matter, ed. by V. Celebonovic, D. Gough, W. Däppen American Institute of Physics Conference Series, vol. 731, 2004, pp. 193-207. doi:10.1063/1.1828405

G. Houdek, Stellar turbulence and mode physics. Astrophysics and Space Science 328, 237-244 
(2010). doi:10.1007/s10509-009-0226-0

G. Houdek, D.O. Gough, Modelling pulsation amplitudes of $\xi$ Hydrae. MNRAS 336, 65-69 (2002). doi:10.1046/j.1365-8711.2002.06024.x

G. Houdek, D.O. Gough, An asteroseismic signature of helium ionization. MNRAS 375, 861880 (2007). doi:10.1111/j.1365-2966.2006.11325.x

G. Houdek, N.J. Balmforth, J. Christensen-Dalsgaard, D.O. Gough, Amplitudes of stochastically excited oscillations in main-sequence stars. Astron. Astrophys. 351, 582-596 (1999)

G. Houdek, W.J. Chaplin, T. Appourchaux, J. Christensen-Dalsgaard, W. Däppen, Y. Elsworth, D.O. Gough, G.R. Isaak, R. New, M.C. Rabello-Soares, Changes in convective properties over the solar cycle: effect on p-mode damping rates. MNRAS 327, 483-487 (2001). doi:10.1046/j.1365-8711.2001.04749.x

R. Howe, D.A. Haber, B.W. Hindman, R. Komm, F. Hill, I. Gonzalez Hernandez, Helioseismic Frequency Shifts in Active Regions, in Subsurface and Atmospheric Influences on Solar Activity, ed. by R. Howe, R.W. Komm, K.S. Balasubramaniam, G.J.D. Petrie Astronomical Society of the Pacific Conference Series, vol. 383, 2008, p. 305

D. Huber, D. Stello, T.R. Bedding, W.J. Chaplin, T. Arentoft, P. Quirion, H. Kjeldsen, Automated extraction of oscillation parameters for Kepler observations of solar-type stars. Communications in Asteroseismology 160, 74 (2009)

D. Huber, T.R. Bedding, D. Stello, B. Mosser, S. Mathur, et al., Asteroseismology of Red Giants from the First Four Months of Kepler Data: Global Oscillation Parameters for 800 Stars. Astrophys. J. 723, 1607-1617 (2010). doi:10.1088/0004-637X/723/2/1607

D. Huber, T.R. Bedding, D. Stello, S. Hekker, et al., Testing Scaling Relations for Solar-like Oscillations from the Main Sequence to Red Giants Using Kepler Data. Astrophys. J. 743, 143 (2011). doi:10.1088/0004-637X/743/2/143

E. Işık, D. Schmitt, M. Schüssler, Magnetic flux generation and transport in cool stars. Astron. Astrophys. 528, 135 (2011). doi:10.1051/0004-6361/201014501

K. Jain, S.C. Tripathy, F. Hill, Solar Activity Phases and Intermediate-Degree Mode Frequencies. Astrophys. J. 695, 1567-1576 (2009). doi:10.1088/0004-637X/695/2/1567

M. Jardine, J.R. Barnes, J.-F. Donati, A. Collier Cameron, The potential magnetic field of AB Doradus: comparison with Zeeman-Doppler images. MNRAS 305, 35-39 (1999). doi:10.1046/j.1365-8711.1999.02621.x

S.J. Jiménez-Reyes, R.A. García, A. Jiménez, W.J. Chaplin, Excitation and Damping of LowDegree Solar p-Modes during Activity Cycle 23: Analysis of GOLF and VIRGO Sun Photometer Data. Astrophys. J. 595, 446-457 (2003). doi:10.1086/377304

S.J. Jiménez-Reyes, W.J. Chaplin, Y. Elsworth, R.A. García, R. Howe, H. Socas-Navarro, T. Toutain, On the Variation of the Peak Asymmetry of Low-l Solar p Modes. Astrophys. J. 654, 1135-1145 (2007). doi:10.1086/509700

C.A. Jones, Planetary Magnetic Fields and Fluid Dynamos. Annual Review of Fluid Mechanics 43, 583-614 (2011). doi:10.1146/annurev-fluid-122109-160727

C.A. Jones, P. Boronski, A.S. Brun, G.A. Glatzmaier, T. Gastine, M.S. Miesch, J. Wicht, Anelastic convection-driven dynamo benchmarks. Icarus 216, 120-135 (2011). doi:10.1016/j.icarus.2011.08.014

L. Jouve, A.S. Brun, On the role of meridional flows in flux transport dynamo models. Astron. Astrophys. 474, 239-250 (2007). doi:10.1051/0004-6361:20077070

L. Jouve, A.S. Brun, Three-Dimensional Nonlinear Evolution of a Magnetic Flux Tube in a Spherical Shell: Influence of Turbulent Convection and Associated Mean Flows. Astrophys. J. 701, 1300-1322 (2009). doi:10.1088/0004-637X/701/2/1300

L. Jouve, B.P. Brown, A.S. Brun, Exploring the $P_{c y c}$ vs. $P_{r o t}$ relation with flux transport dynamo models of solar-like stars. Astron. Astrophys. 509, 32 (2010). doi:10.1051/0004$6361 / 200913103$

L. Jouve, A.S. Brun, G. Aulanier, Global dynamics of subsurface solar active regions. Astrophys. J. 762, 4 (2013). doi:10.1088/0004-637X/762/1/4

P.G. Judge, M.J. Thompson, Solar and Stellar Activity: Diagnostics and Indices, in IAU Symposium, ed. by C.H. Mandrini, D.F. Webb IAU Symposium, vol. 286, 2012, pp. 15-26. doi:10.1017/S1743921312004589

A. Kageyama, T. Sato, Generation mechanism of a dipole field by a magnetohydrodynamic dynamo. Phys. Rev. E 55, 4617-4626 (1997). doi:10.1103/PhysRevE.55.4617

A. Kageyama, T. Sato, "Yin-Yang grid": An overset grid in spherical geometry. Geochemistry, Geophysics, Geosystems 5, 9005 (2004). doi:10.1029/2004GC000734

T. Kallinger, B. Mosser, S. Hekker, D. Huber, et al., Asteroseismology of red giants from the 
first four months of Kepler data: Fundamental stellar parameters. Astron. Astrophys. 522, 1 (2010). doi:10.1051/0004-6361/201015263

P.J. Käpylä, M.J. Mantere, A. Brandenburg, Cyclic Magnetic Activity due to Turbulent Convection in Spherical Wedge Geometry. Astrophys. J. Letters 755, 22 (2012). doi:10.1088/2041-8205/755/1/L22

P.J. Käpylä, M.J. Mantere, A. Brandenburg, Confirmation of bistable stellar differential rotation profiles. ArXiv e-prints (2014)

P.J. Käpylä, M.J. Mantere, G. Guerrero, A. Brandenburg, P. Chatterjee, Reynolds stress and heat flux in spherical shell convection. Astron. Astrophys. 531, 162 (2011). doi:10.1051/0004-6361/201015884

P.J. Käpylä, M.J. Mantere, E. Cole, J. Warnecke, A. Brandenburg, Effects of Enhanced Stratification on Equatorward Dynamo Wave Propagation. Astrophys. J. 778, 41 (2013). doi:10.1088/0004-637X/778/1/41

B.B. Karak, D. Nandy, Turbulent Pumping of Magnetic Flux Reduces Solar Cycle Memory and thus Impacts Predictability of the Sun's Activity. Astrophys. J. Letters 761, 13 (2012). doi:10.1088/2041-8205/761/1/L13

C. Karoff, T.L. Campante, J. Ballot, T. Kallinger, M. Gruberbauer, R.A. Garcia, D.A. Caldwell, J.L. Christiansen, K. Kinemuchi, Observations of intensity fluctuations attributed to granulation and faculae on Sun-like stars from the Kepler mission. ArXiv e-prints (2013)

S.D. Kawaler, Angular momentum loss in low-mass stars. Astrophys. J. 333, 236-247 (1988). doi:10.1086/166740

R. Keppens, K.B. MacGregor, P. Charbonneau, On the evolution of rotational velocity distributions for solar-type stars. Astron. Astrophys. 294, 469-487 (1995)

Y.-C. Kim, P. Demarque, The Theoretical Calculation of the Rossby Number and the "Nonlocal" Convective Overturn Time for Pre-Main-Sequence and Early Post-Main-Sequence Stars. Astrophys. J. 457, 340 (1996). doi:10.1086/176733

K. Kimura, S.-I. Takehiro, M. Yamada, Stability and bifurcation diagram of Boussinesq thermal convection in a moderately rotating spherical shell. Physics of Fluids 23(7), 074101 (2011). doi:10.1063/1.3602917

R. Kippenhahn, A. Weigert, A. Weiss, Stellar Structure and Evolution 2013. doi:10.1007/9783-642-30304-3

L.L. Kitchatinov, S.V. Olemskoy, Differential rotation of main-sequence dwarfs: predicting the dependence on surface temperature and rotation rate. MNRAS 423, 3344-3351 (2012). doi:10.1111/j.1365-2966.2012.21126.x

H. Kjeldsen, T.R. Bedding, Amplitudes of stellar oscillations: the implications for asteroseismology. Astron. Astrophys. 293, 87-106 (1995)

H. Kjeldsen, T.R. Bedding, J. Christensen-Dalsgaard, Correcting Stellar Oscillation Frequencies for Near-Surface Effects. Astrophys. J. Letters 683, 175-178 (2008). doi:10.1086/591667

H. Kjeldsen, T.R. Bedding, M. Viskum, S. Frandsen, Solarlike oscillations in eta Boo. Astron. J. 109, 1313-1319 (1995). doi:10.1086/117363

H. Kjeldsen, T.R. Bedding, I.K. Baldry, H. Bruntt, et al., Confirmation of Solar-like Oscillations in $\eta$ Bootis. Astron. J. 126, 1483-1488 (2003). doi:10.1086/377315

A. Koenigl, Disk accretion onto magnetic T Tauri stars. Astrophys. J. Letters 370, 39-43 (1991). doi:10.1086/185972

A.G. Kosovichev, J.O. Stenflo, Tilt of Emerging Bipolar Magnetic Regions on the Sun. Astrophys. J. Letters 688, 115-118 (2008). doi:10.1086/595619

A. Krishnamurthi, M.H. Pinsonneault, S. Barnes, S. Sofia, Theoretical Models of the Angular Momentum Evolution of Solar-Type Stars. Astrophys. J. 480, 303 (1997). doi:10.1086/303958

J.R. Kuhn, R. Bush, M. Emilio, I.F. Scholl, The Precise Solar Shape and Its Variability. Science 337, 1638 (2012). doi:10.1126/science.1223231

M. Küker, G. Rüdiger, Modelling solar and stellar differential rotation. Journal of Physics Conference Series 118(1), 012029 (2008). doi:10.1088/1742-6596/118/1/012029

N. Lagarde, T. Decressin, C. Charbonnel, P. Eggenberger, S. Ekström, A. Palacios, Thermohaline instability and rotation-induced mixing. III. Grid of stellar models and asymptotic asteroseismic quantities from the pre-main sequence up to the AGB for low- and intermediate-mass stars of various metallicities. Astron. Astrophys. 543, 108 (2012). doi:10.1051/0004-6361/201118331

N.R. Landin, L.T.S. Mendes, L.P.R. Vaz, Theoretical values of convective turnover times and 
Rossby numbers for solar-like, pre-main sequence stars. Astron. Astrophys. 510, 46 (2010). doi:10.1051/0004-6361/200913015

A.F. Lanza, Current State of the Modelling of Photospheric Activity, in Advances in Solid State Physics, ed. by J.C. Suárez, R. Garrido, L.A. Balona, J. Christensen-Dalsgaard Advances in Solid State Physics, vol. 31, 2013, p. 215. doi:10.1007/978-3-642-29630-7

S. Lefebvre, R.A. García, S.J. Jiménez-Reyes, S. Turck-Chièze, S. Mathur, Variations of the solar granulation motions with height using the GOLF/SoHO experiment. Astron. Astrophys. 490, 1143-1149 (2008). doi:10.1051/0004-6361:200810344

R.B. Leighton, Observations of Solar Magnetic Fields in Plage Regions. Astrophys. J. 130, 366 (1959). doi:10.1086/146727

R.B. Leighton, A Magneto-Kinematic Model of the Solar Cycle. Astrophys. J. 156, 1 (1969). doi:10.1086/149943

K.G. Libbrecht, M.F. Woodard, Solar-cycle effects on solar oscillation frequencies. Nature 345, 779-782 (1990). doi:10.1038/345779a0

G.W. Lockwood, B.A. Skiff, G.W. Henry, S. Henry, R.R. Radick, S.L. Baliunas, R.A. Donahue, W. Soon, Patterns of Photometric and Chromospheric Variation among Sun-like Stars: A 20 Year Perspective. Astrophys. J. Supp. 171, 260-303 (2007). doi:10.1086/516752

D. Lynden-Bell, J.P. Ostriker, On the stability of differentially rotating bodies. MNRAS 136 , 293 (1967)

K.B. MacGregor, M. Brenner, Rotational evolution of solar-type stars. I - Main-sequence evolution. Astrophys. J. 376, 204-213 (1991). doi:10.1086/170269

A. Maeder, G. Meynet, Rotating massive stars: From first stars to gamma ray bursts. Reviews of Modern Physics 84, 25-63 (2012). doi:10.1103/RevModPhys.84.25

Z. Magic, R. Collet, M. Asplund, R. Trampedach, W. Hayek, A. Chiavassa, R.F. Stein, A. Nordlund, The Stagger-grid: A grid of 3D stellar atmosphere models. I. Methods and general properties. Astron. Astrophys. 557, 26 (2013). doi:10.1051/0004-6361/201321274

W.V.R. Malkus, M.R.E. Proctor, The macrodynamics of alpha-effect dynamos in rotating fluids. Journal of Fluid Mechanics 67, 417-443 (1975). doi:10.1017/S0022112075000390

E.E. Mamajek, L.A. Hillenbrand, Improved Age Estimation for Solar-Type Dwarfs Using Activity-Rotation Diagnostics. Astrophys. J. 687, 1264-1293 (2008). doi:10.1086/591785

J.P. Marques, M.J. Goupil, Y. Lebreton, S. Talon, A. Palacios, K. Belkacem, R.-M. Ouazzani, B. Mosser, A. Moya, P. Morel, B. Pichon, S. Mathis, J.-P. Zahn, S. Turck-Chièze, P.A.P. Nghiem, Seismic diagnostics for transport of angular momentum in stars. I. Rotational splittings from the pre-main sequence to the red-giant branch. Astron. Astrophys. 549, 74 (2013). doi:10.1051/0004-6361/201220211

S.C. Marsden, P. Petit, S.V. Jeffers, J. Morin, R. Fares, A. Reiners, J.D. do Nascimento Jr., M. Auriere, J. Bouvier, B.D. Carter, C. Catala, B. Dintrans, J.-F. Donati, T. Gastine, M. Jardine, R. Konstantinova-Antova, J. Lanoux, F. Lignieres, A. Morgenthaler, J.C. Ramirez-Velez, S. Theado, V. Van Grootel, the Bcool Collaboration, A Bcool magnetic snapshot survey of solar-type stars. ArXiv e-prints (2013)

M. Martić, J. Schmitt, J.-C. Lebrun, C. Barban, et al., Evidence for global pressure oscillations on Procyon. Astron. Astrophys. 351, 993-1002 (1999)

Y. Masada, K. Yamada, A. Kageyama, Effects of Penetrative Convection on Solar Dynamo. Astrophys. J. 778, 11 (2013). doi:10.1088/0004-637X/778/1/11

J. Mason, D.W. Hughes, S.M. Tobias, The effects of flux transport on interface dynamos. MNRAS 391, 467-480 (2008). doi:10.1111/j.1365-2966.2008.13918.x

S. Mathis, Angular Momentum Transport by Regular Gravito-inertial Waves in Stellar Radiation Zones, in Lecture Notes in Physics, Berlin Springer Verlag, ed. by J.-P. Rozelot, C. Neiner Lecture Notes in Physics, Berlin Springer Verlag, vol. 832, 2011, p. 275

S. Mathis, Angular Momentum Transport by Internal Waves in Stellar Interiors, in EAS Publications Series. EAS Publications Series, vol. 63, 2013a, pp. 269-284. doi:10.1051/eas/1363031

S. Mathis, Transport Processes in Stellar Interiors, in Lecture Notes in Physics, Berlin Springer Verlag, ed. by M. Goupil, K. Belkacem, C. Neiner, F. Lignières, J.J. Green Lecture Notes in Physics, Berlin Springer Verlag, vol. 865, 2013b, p. 23

S. Mathis, J.-P. Zahn, Transport and mixing in the radiation zones of rotating stars. I. Hydrodynamical processes. Astron. Astrophys. 425, 229-242 (2004). doi:10.1051/00046361:20040278

S. Mathis, J.-P. Zahn, Transport and mixing in the radiation zones of rotating stars. II. Axisymmetric magnetic field. Astron. Astrophys. 440, 653-666 (2005). doi:10.1051/0004- 
6361:20052640

S. Mathur, S.J. Jiménez-Reyes, R.A. García, GOLF: A New Proxy for Solar Magnetism, in Solar-Stellar Dynamos as Revealed by Helio- and Asteroseismology: GONG 2008/SOHO 21, ed. by M. Dikpati, T. Arentoft, I. González Hernández, C. Lindsey, F. Hill Astronomical Society of the Pacific Conference Series, vol. 416, 2009, p. 529

S. Mathur, A. Eff-Darwich, R.A. García, S. Turck-Chièze, Sensitivity of helioseismic gravity modes to the dynamics of the solar core. Astron. Astrophys. 484, 517-522 (2008). doi:10.1051/0004-6361:20078839

S. Mathur, R.A. García, C. Régulo, O.L. Creevey, et al., Determining global parameters of the oscillations of solar-like stars. Astron. Astrophys. 511, 46 (2010a). doi:10.1051/0004$6361 / 200913266$

S. Mathur, R.A. García, C. Catala, H. Bruntt, et al., The solar-like CoRoT target HD 170987: spectroscopic and seismic observations. Astron. Astrophys. 518, 53 (2010b). doi:10.1051/0004-6361/201014103

S. Mathur, S. Hekker, R. Trampedach, J. Ballot, et al., Granulation in Red Giants: Observations by the Kepler Mission and Three-dimensional Convection Simulations. Astrophys. J. 741, 119 (2011a). doi:10.1088/0004-637X/741/2/119

S. Mathur, R. Handberg, T.L. Campante, R.A. García, et al., Solar-like Oscillations in KIC 11395018 and KIC 11234888 from 8 Months of Kepler Data. Astrophys. J. 733, 95 (2011b). doi:10.1088/0004-637X/733/2/95

S. Mathur, T.S. Metcalfe, M. Woitaszek, H. Bruntt, et al., A Uniform Asteroseismic Analysis of 22 Solar-type Stars Observed by Kepler. Astrophys. J. 749, 152 (2012). doi:10.1088/0004$637 \mathrm{X} / 749 / 2 / 152$

S. Mathur, R.A. García, A. Morgenthaler, D. Salabert, P. Petit, J. Ballot, C. Régulo, C. Catala, Constraining magnetic-activity modulations in three solar-like stars observed by CoRoT and NARVAL. Astron. Astrophys. 550, 32 (2013a). doi:10.1051/0004-6361/201117913

S. Mathur, H. Bruntt, C. Catala, O. Benomar, et al., Study of HD 169392A observed by CoRoT and HARPS. Astron. Astrophys. 549, 12 (2013b). doi:10.1051/0004-6361/201219678

S. Mathur, R.A. García, J. Ballot, T. Ceillier, D. Salabert, T.S. Metcalfe, C. Régulo, A. Jiménez, S. Bloemen, Magnetic activity of F stars observed by Kepler. Astron. Astrophys. 562, 124 (2014a). doi:10.1051/0004-6361/201322707

S. Mathur, D. Salabert, R.A. García, T. Ceillier, Photometric magnetic-activity metrics tested with the Sun: application to Kepler M dwarfs. Journal of Space Weather and Space Climate 4(27), 15 (2014b). doi:10.1051/swsc/2014011

S.P. Matt, O. Do Cao, B.P. Brown, A.S. Brun, Convection and differential rotation properties of $\mathrm{G}$ and $\mathrm{K}$ stars computed with the ASH code. Astronomische Nachrichten 332, 897 (2011). doi:10.1002/asna.201111624

S.P. Matt, K.B. MacGregor, M.H. Pinsonneault, T.P. Greene, Magnetic Braking Formulation for Sun-like Stars: Dependence on Dipole Field Strength and Rotation Rate. Astrophys. J. Letters 754, 26 (2012). doi:10.1088/2041-8205/754/2/L26

S. Matt, A.S. Brun, A. Strugarek, O. Do Cao, Differential rotation and overshooting in solarlike stars. Astrophys. J., (2014)

A. Mazumdar, M.J.P.F.G. Monteiro, J. Ballot, H.M. Antia, S. Basu, G. Houdek, S. Mathur, M.S. Cunha, V. Silva Aguirre, R.A. García, D. Salabert, G.A. Verner, J. ChristensenDalsgaard, T.S. Metcalfe, D.T. Sanderfer, S.E. Seader, J.C. Smith, W.J. Chaplin, Measurement of Acoustic Glitches in Solar-type Stars from Oscillation Frequencies Observed by Kepler. Astrophys. J. 782, 18 (2014). doi:10.1088/0004-637X/782/1/18

M.E. McIntyre, Magnetic Confinement and the Sharp Tachopause, in The Solar Tachocline, ed. by D.W. Hughes, R. Rosner, N.O. Weiss, 2007, p. 183

A. McQuillan, T. Mazeh, S. Aigrain, Rotation Periods of 34,030 Kepler Main-sequence Stars: The Full Autocorrelation Sample. Astrophys. J. Supp. 211, 24 (2014). doi:10.1088/0067$0049 / 211 / 2 / 24$

S. Meibom, S.A. Barnes, K. Covey, R.D. Jeffries, S. Matt, J. Morin, A. Palacios, A. Reiners, A. Sicilia-Aguilar, J. Irwin, Angular momentum evolution of cool stars: Toward a synthesis of observations and theory before and after the ZAMS. Astronomische Nachrichten 334, 168 (2013). doi:10.1002/asna.201211777

L. Mestel, Magnetic braking by a stellar wind-I. MNRAS 138, 359 (1968)

T.S. Metcalfe, S. Basu, T.J. Henry, D.R. Soderblom, P.G. Judge, M. Knölker, S. Mathur, M. Rempel, Discovery of a 1.6 Year Magnetic Activity Cycle in the Exoplanet Host Star $\iota$ Horologii. Astrophys. J. Letters 723, 213-217 (2010). doi:10.1088/2041-8205/723/2/L213 
T.S. Metcalfe, W.J. Chaplin, T. Appourchaux, R.A. García, et al., Asteroseismology of the Solar Analogs 16 Cyg A and B from Kepler Observations. Astrophys. J. Letters 748, 10 (2012). doi:10.1088/2041-8205/748/1/L10

G. Micela, A. Marino, A comparison between the X-ray variable Sun and solar-like main sequence stars. Astron. Astrophys. 404, 637-643 (2003). doi:10.1051/0004-6361:20030517

M.S. Miesch, Large-Scale Dynamics of the Convection Zone and Tachocline. Living Reviews in Solar Physics 2, 1 (2005). doi:10.12942/lrsp-2005-1

M.S. Miesch, B.P. Brown, Convective Babcock-Leighton Dynamo Models. Astrophys. J. Letters 746, 26 (2012). doi:10.1088/2041-8205/746/2/L26

M.S. Miesch, M. Dikpati, A Three-dimensional Babcock-Leighton Solar Dynamo Model. Astrophys. J. Letters 785, 8 (2014). doi:10.1088/2041-8205/785/1/L8

M.S. Miesch, B.W. Hindman, Gyroscopic Pumping in the Solar Near-surface Shear Layer. Astrophys. J. 743, 79 (2011). doi:10.1088/0004-637X/743/1/79

M.S. Miesch, J. Toomre, Turbulence, Magnetism, and Shear in Stellar Interiors. Annual Review of Fluid Mechanics 41, 317-345 (2009). doi:10.1146/annurev.fluid.010908.165215

M.S. Miesch, A.S. Brun, J. Toomre, Solar Differential Rotation Influenced by Latitudinal Entropy Variations in the Tachocline. Astrophys. J. 641, 618-625 (2006). doi:10.1086/499621

M.S. Miesch, J.R. Elliott, J. Toomre, T.L. Clune, G.A. Glatzmaier, P.A. Gilman, Threedimensional Spherical Simulations of Solar Convection. I. Differential Rotation and Pattern Evolution Achieved with Laminar and Turbulent States. Astrophys. J. 532, 593-615 (2000). doi:10.1086/308555

M.S. Miesch, A.S. Brun, M.L. De Rosa, J. Toomre, Structure and Evolution of Giant Cells in Global Models of Solar Convection. Astrophys. J. 673, 557-575 (2008). doi:10.1086/523838

M.S. Miesch, N.A. Featherstone, M. Rempel, R. Trampedach, On the Amplitude of Convective Velocities in the Deep Solar Interior. Astrophys. J. 757, 128 (2012). doi:10.1088/0004$637 \mathrm{X} / 757 / 2 / 128$

A. Miglio, J. Montalbán, F. Baudin, P. Eggenberger, A. Noels, S. Hekker, J. De Ridder, W. Weiss, A. Baglin, Probing populations of red giants in the galactic disk with CoRoT. Astron. Astrophys. 503, 21-24 (2009). doi:10.1051/0004-6361/200912822

U. Mitra-Kraev, M.J. Thompson, Meridional flow profile measurements with SOHO/MDI. Astronomische Nachrichten 328, 1009-1012 (2007). doi:10.1002/asna.200710873

H.K. Moffatt, Magnetic Field Generation in Electrically Conducting Fluids (Cambridge, Bristol, University, Bristol, England, 1978)

M.J.P.F.G. Monteiro, J. Christensen-Dalsgaard, M.J. Thompson, Seismic study of overshoot at the base of the solar convective envelope. Astron. Astrophys. 283, 247-262 (1994)

B. Montesinos, J.H. Thomas, P. Ventura, I. Mazzitelli, A new look at the relationship between activity, dynamo number and Rossby number in late-type stars. MNRAS 326, 877-884 (2001). doi:10.1046/j.1365-8711.2001.04476.x

E. Moraux, S. Artemenko, J. Bouvier, J. Irwin, M. Ibrahimov, T. Magakian, K. Grankin, E. Nikogossian, C. Cardoso, S. Hodgkin, S. Aigrain, T.A. Movsessian, The Monitor Project: stellar rotation at 13 Myr. I. A photometric monitoring survey of the young open cluster h Persei. Astron. Astrophys. 560, 13 (2013). doi:10.1051/0004-6361/201321508

P. Morel, CESAM: A code for stellar evolution calculations. Astron. Astrophys. Supp. 124, 597-614 (1997). doi:10.1051/aas:1997209

T. Morel, A. Miglio, Assessing the accuracy of the surface gravity determination in latetype stars with solar-like pulsators. MNRAS 419, 34-38 (2012). doi:10.1111/j.17453933.2011.01172.x

A. Morgenthaler, P. Petit, J. Morin, M. Aurière, B. Dintrans, R. Konstantinova-Antova, S. Marsden, Direct observation of magnetic cycles in Sun-like stars. Astronomische Nachrichten 332, 866 (2011a). doi:10.1002/asna.201111592

A. Morgenthaler, P. Petit, J. Morin, M. Aurière, B. Dintrans, Long-term Magnetic Monitoring of 19 Sun-like Stars, in SF2A-2011: Proceedings of the Annual meeting of the French Society of Astronomy and Astrophysics, ed. by G. Alecian, K. Belkacem, R. Samadi, D. Valls-Gabaud, 2011b, pp. 497-501

J. Morin, J.-F. Donati, P. Petit, X. Delfosse, T. Forveille, M.M. Jardine, Large-scale magnetic topologies of late M dwarfs. MNRAS 407, 2269-2286 (2010). doi:10.1111/j.13652966.2010.17101.x

J. Morin, E. Dormy, M. Schrinner, J.-F. Donati, Weak- and strong-field dynamos: from the Earth to the stars. MNRAS 418, 133-137 (2011). doi:10.1111/j.1745-3933.2011.01159.x 
J. Morin, M. Jardine, A. Reiners, D. Shulyak, B. Beeck, G. Hallinan, L. Hebb, G. Hussain, S.V. Jeffers, O. Kochukhov, A. Vidotto, L. Walkowicz, Multiple views of magnetism in cool stars. Astronomische Nachrichten 334, 48 (2013). doi:10.1002/asna.201211771

D. Moss, J. Brooke, Towards a model for the solar dynamo. MNRAS 315, 521-533 (2000). doi:10.1046/j.1365-8711.2000.03452.x

B. Mosser, T. Appourchaux, On detecting the large separation in the autocorrelation of stellar oscillation times series. Astron. Astrophys. 508, 877-887 (2009). doi:10.1051/0004$6361 / 200912944$

B. Mosser, F. Bouchy, M. Martić, T. Appourchaux, et al., Asteroseismology of Procyon with SOPHIE. Astron. Astrophys. 478, 197-202 (2008). doi:10.1051/0004-6361:20078337

B. Mosser, F. Baudin, A.F. Lanza, J.C. Hulot, C. Catala, A. Baglin, M. Auvergne, Short-lived spots in solar-like stars as observed by CoRoT. Astron. Astrophys. 506, 245-254 (2009). doi:10.1051/0004-6361/200911942

B. Mosser, K. Belkacem, M. Goupil, A. Miglio, et al., Red-giant seismic properties analyzed with CoRoT. Astron. Astrophys. 517, 22 (2010). doi:10.1051/0004-6361/201014036

B. Mosser, M.J. Goupil, K. Belkacem, E. Michel, D. Stello, et al., Probing the core structure and evolution of red giants using gravity-dominated mixed modes observed with Kepler. Astron. Astrophys. 540, 143 (2012). doi:10.1051/0004-6361/201118519

B. Mosser, E. Michel, K. Belkacem, M.J. Goupil, A. Baglin, C. Barban, J. Provost, R. Samadi, M. Auvergne, C. Catala, Asymptotic and measured large frequency separations. Astron. Astrophys. 550, 126 (2013). doi:10.1051/0004-6361/201220435

K. Mursula, B. Zieger, J.H. Vilppola, Mid-term quasi-periodicities in geomagnetic activity during the last 15 solar cycles: Connection to solar dynamo strength To the memory of Karolen I. Paularena (1957-2001). Solar Phys. 212, 201-207 (2003). doi:10.1023/A:1022980029618

D. Nandy, Exploring Magnetic Activity from The Sun to the Stars. Solar Phys. 224, 161-169 (2004). doi:10.1007/s11207-005-4990-x

D. Nandy, A.R. Choudhuri, Explaining the Latitudinal Distribution of Sunspots with Deep Meridional Flow. Science 296, 1671-1673 (2002). doi:10.1126/science.1070955

D. Nandy, A. Muñoz-Jaramillo, P.C.H. Martens, The Unusual Minimum of sunspot cycle 23 caused by meridional plasma flow variations. Nature 471, 80-82 (2011). doi:10.1038/nature09786

N.J. Nelson, B.P. Brown, A.S. Brun, M.S. Miesch, J. Toomre, Buoyant Magnetic Loops in a Global Dynamo Simulation of a Young Sun. Astrophys. J. Letters 739, 38 (2011). doi:10.1088/2041-8205/739/2/L38

N.J. Nelson, B.P. Brown, A.S. Brun, M.S. Miesch, J. Toomre, Magnetic Wreaths and Cycles in Convective Dynamos. Astrophys. J. 762, 73 (2013). doi:10.1088/0004-637X/762/2/73

N.J. Nelson, B.P. Brown, A. Sacha Brun, M.S. Miesch, J. Toomre, Buoyant Magnetic Loops Generated by Global Convective Dynamo Action. Solar Phys. 289, 441-458 (2014). doi:10.1007/s11207-012-0221-4

M.B. Nielsen, L. Gizon, H. Schunker, C. Karoff, Rotation periods of 12000 main-sequence Kepler stars: Dependence on stellar spectral type and comparison with v sin i observations. Astron. Astrophys. 557, 10 (2013). doi:10.1051/0004-6361/201321912

N. Nishikawa, K. Kusano, Simulation study of the symmetry-breaking instability and the dipole field reversal in a rotating spherical shell dynamo. Physics of Plasmas 15(8), 082903 (2008). doi:10.1063/1.2959120

R.W. Noyes, N.O. Weiss, A.H. Vaughan, The relation between stellar rotation rate and activity cycle periods. Astrophys. J. 287, 769-773 (1984a). doi:10.1086/162735

R.W. Noyes, L.W. Hartmann, S.L. Baliunas, D.K. Duncan, A.H. Vaughan, Rotation, convection, and magnetic activity in lower main-sequence stars. Astrophys. J. 279, 763-777 (1984b). doi:10.1086/161945

R.L.F. Oglethorpe, P. Garaud, Spin-down Dynamics of Magnetized Solar-type Stars. Astrophys. J. 778, 166 (2013). doi:10.1088/0004-637X/778/2/166

K. Oláh, Z. Kolláth, T. Granzer, K.G. Strassmeier, A.F. Lanza, S. Järvinen, H. Korhonen, S.L. Baliunas, W. Soon, S. Messina, G. Cutispoto, Multiple and changing cycles of active stars. II. Results. Astron. Astrophys. 501, 703-713 (2009). doi:10.1051/0004-6361/200811304

A. Palacios, S. Talon, C. Charbonnel, M. Forestini, Rotational mixing in low-mass stars. I Effect of the mu-gradients in main sequence and subgiant Pop I stars. Astron. Astrophys. 399, 603-616 (2003). doi:10.1051/0004-6361:20021759

P.L. Pallé, C. Régulo, T. Roca Cortés, Solar cycle induced variations of the low L solar acoustic spectrum. Astron. Astrophys. 224, 253-258 (1989) 
E.N. Parker, Hydromagnetic Dynamo Models. Astrophys. J. 122, 293 (1955). doi:10.1086/146087

J. Pedlosky, Geophysical Fluid Dynamics 1982

P. Petit, B. Dintrans, S.K. Solanki, J.-F. Donati, M. Aurière, et al., Toroidal versus poloidal magnetic fields in Sun-like stars: a rotation threshold. MNRAS 388, 80-88 (2008). doi:10.1111/j.1365-2966.2008.13411.x

A.A. Pevtsov, G.H. Fisher, L.W. Acton, D.W. Longcope, C.M. Johns-Krull, C.C. Kankelborg, T.R. Metcalf, The Relationship Between X-Ray Radiance and Magnetic Flux. Astrophys. J. 598, 1387-1391 (2003). doi:10.1086/378944

M. Pinsonneault, Mixing in Stars. Annual Review of Astronomy and Astrophysics 35, 557-605 (1997). doi:10.1146/annurev.astro.35.1.557

M.H. Pinsonneault, S.D. Kawaler, P. Demarque, Rotation of low-mass stars - A new probe of stellar evolution. Astrophys. J. Supp. 74, 501-550 (1990). doi:10.1086/191507

M.H. Pinsonneault, S.D. Kawaler, S. Sofia, P. Demarque, Evolutionary models of the rotating sun. Astrophys. J. 338, 424-452 (1989). doi:10.1086/167210

R.F. Pinto, A.S. Brun, Flux Emergence in a Magnetized Convection Zone. Astrophys. J. 772, 55 (2013). doi:10.1088/0004-637X/772/1/55

R.F. Pinto, A.S. Brun, L. Jouve, R. Grappin, Coupling the Solar Dynamo and the Corona: Wind Properties, Mass, and Momentum Losses during an Activity Cycle. Astrophys. J. 737, 72 (2011). doi:10.1088/0004-637X/737/2/72

N. Pizzolato, A. Maggio, G. Micela, S. Sciortino, P. Ventura, The stellar activity-rotation relationship revisited: Dependence of saturated and non-saturated X-ray emission regimes on stellar mass for late-type dwarfs. Astron. Astrophys. 397, 147-157 (2003). doi:10.1051/0004-6361:20021560

K. Poppenhäger, J. Robrade, J.H.M.M. Schmitt, J.C. Hall, 51 Pegasi - a planet-bearing Maunder minimum candidate. Astron. Astrophys. 508, 1417-1421 (2009). doi:10.1051/0004$6361 / 200912945$

A. Pouquet, U. Frisch, J. Leorat, Strong MHD helical turbulence and the nonlinear dynamo effect. Journal of Fluid Mechanics 77, 321-354 (1976). doi:10.1017/S0022112076002140

É. Racine, P. Charbonneau, M. Ghizaru, A. Bouchat, P.K. Smolarkiewicz, On the Mode of Dynamo Action in a Global Large-eddy Simulation of Solar Convection. Astrophys. J. 735, 46 (2011). doi:10.1088/0004-637X/735/1/46

H. Rauer, C. Catala, C. Aerts, T. Appourchaux, W. Benz, A. Brandeker, J. ChristensenDalsgaard, M. Deleuil, L. Gizon, M.-J. Goupil, M. Güdel, E. Janot-Pacheco, M. Mas-Hesse, I. Pagano, G. Piotto, D. Pollacco, N.C. Santos, A. Smith, J. -C., Suárez, R. Szabó, S. Udry, V. Adibekyan, Y. Alibert, J.-M. Almenara, P. Amaro-Seoane, M. Ammler-von Eiff, M. Asplund, E. Antonello, W. Ball, S. Barnes, F. Baudin, K. Belkacem, M. Bergemann, G. Bihain, A.C. Birch, X. Bonfils, I. Boisse, A.S. Bonomo, F. Borsa, I.M. Brandão, E. Brocato, S. Brun, M. Burleigh, R. Burston, J. Cabrera, S. Cassisi, W. Chaplin, S. Charpinet, C. Chiappini, R.P. Church, S. Csizmadia, M. Cunha, M. Damasso, M.B. Davies, H.J. Deeg, R.F. Díaz, S. Dreizler, C. Dreyer, P. Eggenberger, D. Ehrenreich, P. Eigmüller, A. Erikson, R. Farmer, S. Feltzing, F. de Oliveira Fialho, P. Figueira, T. Forveille, M. Fridlund, R.A. García, P. Giommi, G. Giuffrida, M. Godolt, J. Gomes da Silva, T. Granzer, J.L. Grenfell, A. Grotsch-Noels, E. Günther, C.A. Haswell, A.P. Hatzes, G. Hébrard, S. Hekker, R. Helled, K. Heng, J.M. Jenkins, A. Johansen, M.L. Khodachenko, K.G. Kislyakova, W. Kley, U. Kolb, N. Krivova, F. Kupka, H. Lammer, A.F. Lanza, Y. Lebreton, D. Magrin, P. Marcos-Arenal, P.M. Marrese, J.P. Marques, J. Martins, S. Mathis, S. Mathur, S. Messina, A. Miglio, J. Montalban, M. Montalto, M.J.P.F.G. Monteiro, H. Moradi, E. Moravveji, C. Mordasini, T. Morel, A. Mortier, V. Nascimbeni, R.P. Nelson, M.B. Nielsen, L. Noack, A.J. Norton, A. Ofir, M. Oshagh, R.-M. Ouazzani, P. Pápics, V.C. Parro, P. Petit, B. Plez, E. Poretti, A. Quirrenbach, R. Ragazzoni, G. Raimondo, M. Rainer, D.R. Reese, R. Redmer, S. Reffert, B. Rojas-Ayala, I.W. Roxburgh, S. Salmon, A. Santerne, J. Schneider, J. Schou, S. Schuh, H. Schunker, A. Silva-Valio, R. Silvotti, I. Skillen, I. Snellen, F. Sohl, S.G. Sousa, A. Sozzetti, D. Stello, K.G. Strassmeier, M. Švanda, G.M. Szabó, A. Tkachenko, D. Valencia, V. van Grootel, S.D. Vauclair, P. Ventura, F.W. Wagner, N.A. Walton, J. Weingrill, S.C. Werner, P.J. Wheatley, K. Zwintz, The PLATO 2.0 Mission. ArXiv e-prints (2013)

L.M. Rebull, J.R. Stauffer, S.T. Megeath, J.L. Hora, L. Hartmann, A Correlation between Pre-Main-Sequence Stellar Rotation Rates and IRAC Excesses in Orion. Astrophys. J. 646, 297-303 (2006). doi:10.1086/504865 
A. Reiners, Observations of Cool-Star Magnetic Fields. Living Reviews in Solar Physics 9, 1 (2012). doi:10.12942/lrsp-2012-1

A. Reiners, S. Mohanty, Radius-dependent Angular Momentum Evolution in Low-mass Stars. I. Astrophys. J. 746, 43 (2012). doi:10.1088/0004-637X/746/1/43

T. Reinhold, A. Reiners, G. Basri, Rotation and differential rotation of active Kepler stars. ArXiv e-prints (2013)

V. Reville, A.S. Brun, S. Matt, A. Strugarek, R. Pinto, The Effect of Magnetic Topology on Thermally-Driven Wind: Towards a General Formulation of the Braking Law. Astrophys. J.in press (2014)

M. Rieutord, F. Rincon, The Sun's Supergranulation. Living Reviews in Solar Physics 7, 2 (2010). doi:10.12942/lrsp-2010-2

R.S. Ronan, K. Cadora, B.J. Labonte, Solar cycle changes in the high frequency spectrum. Solar Phys. 150, 389-392 (1994). doi:10.1007/BF00712900

C.S. Rosenthal, J. Christensen-Dalsgaard, G. Houdek, M.J.P.F.G. Monteiro, A. Nordlund, R. Trampedach, Seismology of the Solar Surface Regions, in Helioseismology. ESA Special Publication, vol. 376, 1995, pp. 459-464

G. Ruediger, L.L. Kitchatinov, The Internal Solar Rotation in Its Spin-down History. Astrophys. J. 466, 1078 (1996). doi:10.1086/177577

S. Saar, Stellar Dynamos: Scaling Laws and Coronal Connections, in Stellar Coronae in the Chandra and XMM-NEWTON Era, ed. by F. Favata, J.J. Drake Astronomical Society of the Pacific Conference Series, vol. 277, 2002, p. 311

S.H. Saar, Starspots, Cycles, and Magnetic Fields, in IAU Symposium, ed. by D. Prasad Choudhary, K.G. Strassmeier IAU Symposium, vol. 273, 2011, pp. 61-67. doi:10.1017/S1743921311015018

S.H. Saar, A. Brandenburg, Time Evolution of the Magnetic Activity Cycle Period. II. Results for an Expanded Stellar Sample. Astrophys. J. 524, 295-310 (1999). doi:10.1086/307794

D. Salabert, C. Régulo, J. Ballot, R.A. García, S. Mathur, About the p-mode frequency shifts in HD 49933. Astron. Astrophys. 530, 127 (2011a). doi:10.1051/0004-6361/201116633

D. Salabert, R.A. García, P.L. Pallé, A. Jiménez, Global p-mode oscillations throughout the complete solar cycle 23 and the beginning of cycle 24. Journal of Physics Conference Series 271(1), 012030 (2011b). doi:10.1088/1742-6596/271/1/012030

R. Samadi, K. Belkacem, H.-G. Ludwig, E. Caffau, T.L. Campante, et al. , Stellar granulation as seen in disk-integrated intensity. II. Theoretical scaling relations compared with observations. Astron. Astrophys. 559, 40 (2013). doi:10.1051/0004-6361/201220817

L. Sbordone, P. Bonifacio, E. Caffau, H.-G. Ludwig, N.T. Behara, J.I. González Hernández, M. Steffen, R. Cayrel, B. Freytag, C. van't Veer, P. Molaro, B. Plez, T. Sivarani, M. Spite, F. Spite, T.C. Beers, N. Christlieb, P. François, V. Hill, The metal-poor end of the Spite plateau. I. Stellar parameters, metallicities, and lithium abundances. Astron. Astrophys. 522, 26 (2010). doi:10.1051/0004-6361/200913282

J. Schou, D.L. Buzasi, Observations of P-modes in $\alpha$ cen, in SOHO 10/GONG 2000 Workshop: Helio- and Asteroseismology at the Dawn of the Millennium, ed. by A. Wilson \& P. L. Pallé ESA Special Publication, vol. 464, 2001, pp. 391-394

J. Schou, H.M. Antia, S. Basu, R.S. Bogart, R.I. Bush, et al. , Helioseismic Studies of Differential Rotation in the Solar Envelope by the Solar Oscillations Investigation Using the Michelson Doppler Imager. Astrophys. J. 505, 390-417 (1998). doi:10.1086/306146

C.J. Schrijver, A.K. Dobson, R.R. Radick, The magnetic, basal, and radiative-equilibrium components in Mount Wilson CA II H + K fluxes. Astrophys. J. 341, 1035-1044 (1989). doi:10.1086/167561

C.J. Schrijver, J. Beer, U. Baltensperger, E.W. Cliver, M. Güdel, et al. , Estimating the frequency of extremely energetic solar events, based on solar, stellar, lunar, and terrestrial records. Journal of Geophysical Research (Space Physics) 117, 8103 (2012). doi:10.1029/2012JA017706

M. Schrinner, L. Petitdemange, E. Dormy, Dipole Collapse and Dynamo Waves in Global Direct Numerical Simulations. Astrophys. J. 752, 121 (2012). doi:10.1088/0004$637 \mathrm{X} / 752 / 2 / 121$

M. Schrinner, L. Petitdemange, R. Raynaud, E. Dormy, Topology and field strength in spherical, anelastic dynamo simulations. Astron. Astrophys. 564, 78 (2014). doi:10.1051/00046361/201322801

G. Schubert, K. Zhang, M.G. Kivelson, J.D. Anderson, The magnetic field and internal structure of Ganymede. Nature 384, 544-545 (1996). doi:10.1038/384544a0 
A.I. Shapiro, S.K. Solanki, N.A. Krivova, W.K. Schmutz, W.T. Ball, R. Knaack, E.V. Rozanov, Y.C. Unruh, The variability of Sun-like stars: reproducing observed photometric trends. ArXiv e-prints (2014)

H. Shapley, On the Nature and Cause of Cepheid Variation. Astrophys. J. 40, 448 (1914). doi:10.1086/142137

F. Shu, J. Najita, E. Ostriker, F. Wilkin, S. Ruden, S. Lizano, Magnetocentrifugally driven flows from young stars and disks. 1: A generalized model. Astrophys. J. 429, 781-796 (1994). doi:10.1086/174363

H. Siedentopf, Konvektion in Sternatmosphären. Astronomische Nachrichten 247, 297 (1933)

V. Silva Aguirre, L. Casagrande, S. Basu, T.L. Campante, et al., Verifying asteroseismically determined parameters of Kepler stars using hipparcos parallaxes: self-consistent stellar properties and distances. ArXiv e-prints (2012)

R.D. Simitev, F.H. Busse, Bistability and hysteresis of dipolar dynamos generated by turbulent convection in rotating spherical shells. EPL (Europhysics Letters) 85, 19001 (2009). doi:10.1209/0295-5075/85/19001

R. Simoniello, K. Jain, S.C. Tripathy, S. Turck-Chièze, C. Baldner, W. Finsterle, F. Hill, M. Roth, The Quasi-biennial Periodicity as a Window on the Solar Magnetic Dynamo Configuration. Astrophys. J. 765, 100 (2013). doi:10.1088/0004-637X/765/2/100

A. Skumanich, Time Scales for CA II Emission Decay, Rotational Braking, and Lithium Depletion. Astrophys. J. 171, 565 (1972). doi:10.1086/151310

P.K. Smolarkiewicz, P. Charbonneau, EULAG, a computational model for multiscale flows: An MHD extension. Journal of Computational Physics 236, 608-623 (2013). doi:10.1016/j.jcp.2012.11.008

W.H. Soon, S.L. Baliunas, Q. Zhang, An interpretation of cycle periods of stellar chromospheric activity. Astrophys. J. Letters 414, 33-36 (1993). doi:10.1086/186989

F. Spada, A.C. Lanzafame, A.F. Lanza, A semi-analytic approach to angular momentum transport in stellar radiative interiors. MNRAS 404, 641-660 (2010). doi:10.1111/j.13652966.2010.16325.x

E.A. Spiegel, J.-P. Zahn, The solar tachocline. Astron. Astrophys. 265, 106-114 (1992)

M. Spite, F. Spite, P. Bonifacio, The cosmic lithium problem . an observer's perspective. Memorie della Societa Astronomica Italiana Supplementi 22, 9 (2012)

H. Spruit, Theory of solar irradiance variations. Space Sci. Rev. 94, 113-126 (2000)

H.C. Spruit, Dynamo action by differential rotation in a stably stratified stellar interior. Astron. Astrophys. 381, 923-932 (2002). doi:10.1051/0004-6361:20011465

B. Sreenivasan, C.A. Jones, The role of inertia in the evolution of spherical dynamos. Geophysical Journal International 164, 467-476 (2006). doi:10.1111/j.1365-246X.2005.02845.x

J.R. Stauffer, L.W. Hartmann, The distribution of rotational velocities for low-mass stars in the Pleiades. Astrophys. J. 318, 337-355 (1987). doi:10.1086/165371

R.F. Stein, A. Nordlund, Convection and Its Influence on Oscillations, in Challenges to Theories of the Structure of Moderate-Mass Stars, ed. by D. Gough, J. Toomre Lecture Notes in Physics, Berlin Springer Verlag, vol. 388, 1991, p. 195

D. Stello, W.J. Chaplin, S. Basu, Y. Elsworth, T.R. Bedding, The relation between $\Delta \nu$ and $\nu_{\max }$ for solar-like oscillations. MNRAS 400, 80-84 (2009). doi:10.1111/j.17453933.2009.00767.x

S.-I. Takehiro, Y.-Y. Hayashi, Mean zonal flows excited by critical thermal convection in rotating spherical shells. Geophysical and Astrophysical Fluid Dynamics 90, 43-77 (1999). doi:10.1080/03091929908203692

S. Talon, C. Charbonnel, Angular momentum transport by internal gravity waves. II. Pop II stars from the Li plateau to the horizontal branch. Astron. Astrophys. 418, 1051-1060 (2004). doi:10.1051/0004-6361:20034334

M.J. Thompson, A new inversion of solar rotational splitting data. Solar Phys. 125, 1-12 (1990). doi:10.1007/BF00154773

M.J. Thompson, J. Christensen-Dalsgaard, M.S. Miesch, J. Toomre, The Internal Rotation of the Sun. Annual Review of Astronomy and Astrophysics 41, 599-643 (2003). doi:10.1146/annurev.astro.41.011802.094848

M.J. Thompson, A.S. Brun, J.L. Culhane, L. Gizon, M. Roth, T. Sekii, Helioseismology and dynamics of the Solar Interior. ISSI Space Science Series in press

A.O. Thygesen, S. Frandsen, H. Bruntt, T. Kallinger, et al., Atmospheric parameters of 82 red giants in the Kepler field. Astron. Astrophys. 543, 160 (2012). doi:10.1051/0004$6361 / 201219237$ 
S.M. Tobias, The solar cycle: parity interactions and amplitude modulation. Astron. Astrophys. 322, 1007-1017 (1997)

S.M. Tobias, Relating stellar cycle periods to dynamo calculations. MNRAS 296, 653-661 (1998). doi:10.1046/j.1365-8711.1998.01412.x

R. Trampedach, R.F. Stein, The Mass Mixing Length in Convective Stellar Envelopes. Astrophys. J. 731, 78 (2011). doi:10.1088/0004-637X/731/2/78

S. Turck-Chièze, S. Couvidat, A.G. Kosovichev, A.H. Gabriel, et al., Solar Neutrino Emission Deduced from a Seismic Model. Astrophys. J. Letters 555, 69-73 (2001). doi:10.1086/321726

R.K. Ulrich, Determination of stellar ages from asteroseismology. Astrophys. J. Letters 306, 37-40 (1986). doi:10.1086/184700

J.L. van Saders, M.H. Pinsonneault, Fast Star, Slow Star; Old Star, Young Star: Subgiant Rotation as a Population and Stellar Physics Diagnostic. Astrophys. J. 776, 67 (2013). doi:10.1088/0004-637X/776/2/67

G.M. Vasil, D. Lecoanet, B.P. Brown, T.S. Wood, E.G. Zweibel, Energy Conservation and Gravity Waves in Sound-proof Treatments of Stellar Interiors. II. Lagrangian Constrained Analysis. Astrophys. J. 773, 169 (2013). doi:10.1088/0004-637X/773/2/169

A.A. Vidotto, S.G. Gregory, M. Jardine, J.-F. Donati, P. Petit, J. Morin, C.P. Folsom, J. Bouvier, A.C. Cameron, G. Hussain, S. Marsden, I.A. Waite, R. Fares, S. Jeffers, J.D. do Nascimento Jr, Stellar magnetism: empirical trends with age and rotation. ArXiv e-prints (2014)

F.L. Villante, A.M. Serenelli, F. Delahaye, M.H. Pinsonneault, The Chemical Composition of the Sun from Helioseismic and Solar Neutrino Data. Astrophys. J. 787, 13 (2014). doi:10.1088/0004-637X/787/1/13

S.N. Vogel, L.V. Kuhi, Rotational velocities of pre-main-sequence stars. Astrophys. J. 245, 960-976 (1981). doi:10.1086/158872

A. Vögler, M. Schüssler, A solar surface dynamo. Astron. Astrophys. 465, 43-46 (2007). doi:10.1051/0004-6361:20077253

S.V. Vorontsov, A Search of the Effects of Magnetic Field in the Solar 5-minute Oscillations, in Advances in Helio- and Asteroseismology, ed. by J. Christensen-Dalsgaard, S. Frandsen IAU Symposium, vol. 123, 1988, p. 151

L.M. Walkowicz, G.S. Basri, Rotation periods, variability properties and ages for Kepler exoplanet candidate host stars. MNRAS 436, 1883-1895 (2013). doi:10.1093/mnras/stt1700

J. Warnecke, P.J. Käpylä, M.J. Mantere, A. Brandenburg, Spoke-like Differential Rotation in a Convective Dynamo with a Coronal Envelope. Astrophys. J. 778, 141 (2013). doi:10.1088/0004-637X/778/2/141

E.J. Weber, L. Davis Jr., The Angular Momentum of the Solar Wind. Astrophys. J. 148, 217-227 (1967). doi:10.1086/149138

M.A. Weber, Y. Fan, M.S. Miesch, The Rise of Active Region Flux Tubes in the Turbulent Solar Convective Envelope. Astrophys. J. 741, 11 (2011). doi:10.1088/0004-637X/741/1/11

M.A. Weber, Y. Fan, M.S. Miesch, Comparing Simulations of Rising Flux Tubes Through the Solar Convection Zone with Observations of Solar Active Regions: Constraining the Dynamo Field Strength. Solar Phys. 287, 239-263 (2013). doi:10.1007/s11207-012-0093-7

N.O. Weiss, Solar and Stellar Dynamos, in Lectures on Solar and Planetary Dynamos, ed. by M.R.E. Proctor, A.D. Gilbert, 1994, p. 59

T.R. White, T.R. Bedding, M. Gruberbauer, O. Benomar, D. Stello, T. Appourchaux, W.J. Chaplin, J. Christensen-Dalsgaard, Y.P. Elsworth, R.A. García, S. Hekker, D. Huber, H. Kjeldsen, B. Mosser, K. Kinemuchi, F. Mullally, M. Still, Solving the Mode Identification Problem in Asteroseismology of F Stars Observed with Kepler. Astrophys. J. Letters 751, 36 (2012). doi:10.1088/2041-8205/751/2/L36

O.C. Wilson, Chromospheric variations in main-sequence stars. Astrophys. J. 226, 379-396 (1978a). doi:10.1086/156618

O.C. Wilson, Chromospheric variations in main-sequence stars. Astrophys. J. 226, 379-396 (1978b). doi:10.1086/156618

B.E. Wood, Astrospheres and Solar-like Stellar Winds. Living Reviews in Solar Physics 1, 2 (2004). doi:10.12942/lrsp-2004-2

M.F. Woodard, R.W. Noyes, Change of solar oscillation eigenfrequencies with the solar cycle. Nature 318, 449-450 (1985). doi:10.1038/318449a0

J.T. Wright, Do We Know of Any Maunder Minimum Stars? Astron. J. 128, 1273-1278 (2004). doi:10.1086/423221 
J.T. Wright, G.W. Marcy, R.P. Butler, S.S. Vogt, Chromospheric Ca II Emission in Nearby F, G, K, and M Stars. Astrophys. J. Supp. 152, 261-295 (2004). doi:10.1086/386283

N.J. Wright, J.J. Drake, E.E. Mamajek, G.W. Henry, The Stellar-activity-Rotation Relationship and the Evolution of Stellar Dynamos. Astrophys. J. 743, 48 (2011). doi:10.1088/0004$637 \mathrm{X} / 743 / 1 / 48$

A.R. Yeates, D. Nandy, D.H. Mackay, Exploring the Physical Basis of Solar Cycle Predictions: Flux Transport Dynamics and Persistence of Memory in Advection- versus Diffusion-dominated Solar Convection Zones. Astrophys. J. 673, 544-556 (2008). doi:10.1086/524352

M. Yoshida, A. Kageyama, Application of the Yin-Yang grid to a thermal convection of a Boussinesq fluid with infinite Prandtl number in a three-dimensional spherical shell. Geophys. Res. Lett. 31, 12609 (2004). doi:10.1029/2004GL019970

J.-P. Zahn, Convective penetration in stellar interiors. Astron. Astrophys. 252, 179-188 (1991)

J.-P. Zahn, Circulation and turbulence in rotating stars. Astron. Astrophys. 265, 115-132 (1992)

M. Zhang, K. Penev, Stars Get Dizzy After Lunch. Astrophys. J. 787, 131 (2014). doi:10.1088/0004-637X/787/2/131

J. Zhao, R.S. Bogart, A.G. Kosovichev, T.L. Duvall Jr., T. Hartlep, Detection of Equatorward Meridional Flow and Evidence of Double-cell Meridional Circulation inside the Sun. Astrophys. J. Letters 774, 29 (2013). doi:10.1088/2041-8205/774/2/L29 